Supporting information for:

\title{
Unexpected Formation of Aryl Ketones by Palladium-Catalyzed Coupling of Aryl Bromides with Vinylic Acetates
}

\author{
Mickaël Jean, ${ }^{\S}$ Jacques Renault, ${ }^{\S}$ Philippe Uriac, ${ }^{*}$ Marc Capet, ${ }^{\dagger}$ Pierre van de Weghe ${ }^{*}{ }^{\ddagger}$ \\ ${ }^{\S}$ EA Substances Lichéniques et Photoprotection, Faculté de Pharmacie, Université de Rennes 1, 2 avenue du \\ Professeur Léon Bernard, F-35043 Rennes Cedex, France, ${ }^{\dagger}$ Bioprojet-Biotech, 4 rue du Chesnay Beauregard, \\ BP96205, F-35762 Saint-Grégoire, France and Laboratoire de Chimie Organique et Bioorganique associé au \\ CNRS, ENSC-Mu, Université de Haute Alsace, 3 rue A. Werner, F-68093 Mulhouse Cedex, France.
}

E-mail: philippe.uriac@univ-rennes1.fr; pierre.van-de-weghe@univ-rennes1.fr

\section{Contents:}

1. General informations

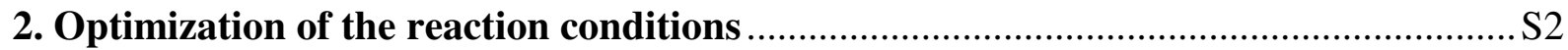

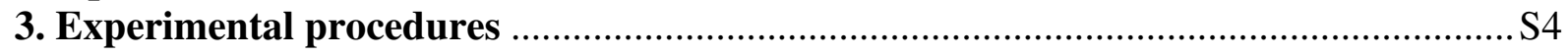

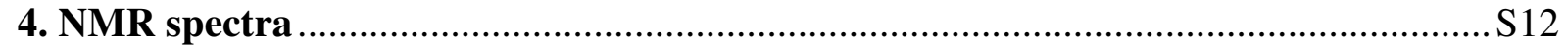

We gratefully acknowledge the CRMPO for mass measurements, S. Sinbandhit for ${ }^{1}$ D NMR and I. Morel for GC-MS technical assistance.

\section{General informations}

${ }^{1} \mathrm{H}$ NMR and ${ }^{13} \mathrm{C}$ NMR spectra were recorded on a JEOL JNM-GSX270 spectrometer in $\mathrm{CDCl}_{3}$ using tetramethylsilane as the internal standard. Data are reported as fellows: chemical shift in ppm $(\delta)$, multiplicity $(\mathrm{s}=$ singlet, $\mathrm{d}=$ doublet, $\mathrm{t}=$ triplet, $\mathrm{q}=$ quartet, $\mathrm{qt}=$ quintet, $\mathrm{dd}=$ doublet of doublets, $\mathrm{dt}=$ doublet of triplets, $\mathrm{tt}=$ triplet of triplets and $\mathrm{m}=$ multiplet), coupling constant (Hz), integration. GC analyses were performed on a Chrompack CP9002 using a CPSIL 5CB capillary column $(10 \mathrm{~m} \times 0.25 \mathrm{~mm}$, Varian $)$. HRMS were measured on a Varian MAT311 spectrometer. Melting points were obtained on a hot bench from Heizbank. All reagents were obtained from commercial suppliers and were used as received. Toluene and $N, N$-dimethylformamide were distilled over calcium hydride. 


\section{Optimization of the reaction conditions}

2.1 Effect of the solvent

\begin{tabular}{|c|c|c|c|c|c|c|c|}
\hline (1 equiv) & 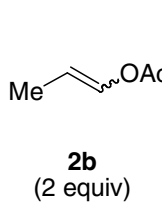 & 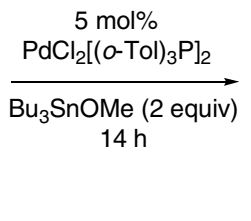 & 3d & f & & 6 & 7 \\
\hline \multirow{2}{*}{ Entry } & \multirow{2}{*}{ Solvent } & \multirow{2}{*}{ Temperature } & \multirow{2}{*}{$\begin{array}{c}\text { Conversion } \\
(\%)\end{array}$} & \multicolumn{4}{|c|}{ Yields (\%) } \\
\hline & & & & 3d & 5 & 6 & 7 \\
\hline 1 & DMSO & r.t. & 14 & 10 & 2 & 1 & traces \\
\hline 2 & DMSO & $60^{\circ} \mathrm{C}$ & 79 & 39 & 18 & 10 & 3 \\
\hline 3 & DMSO & $100^{\circ} \mathrm{C}$ & 100 & 49 & 11 & 11 & 7 \\
\hline 4 & DMF & r.t. & 41 & 24 & 4 & 4 & 3 \\
\hline 5 & DMF & $60^{\circ} \mathrm{C}$ & 85 & 31 & 19 & 10 & 6 \\
\hline 6 & DMF & $100^{\circ} \mathrm{C}$ & 100 & 36 & 10 & 9 & 10 \\
\hline 7 & PhMe & r.t. & 4 & 2 & traces & traces & traces \\
\hline 8 & PhMe & $60^{\circ} \mathrm{C}$ & 65 & 40 & 6 & 2 & 1 \\
\hline 9 & PhMe & $100^{\circ} \mathrm{C}$ & 100 & 44 & 11 & 13 & 8 \\
\hline
\end{tabular}

Best conversion and yield were observed in DMSO at $100{ }^{\circ} \mathrm{C}$. At lower temperature the reaction was incomplete after $14 \mathrm{~h}$.

2.2 Study of palladium source and ligand phosphine

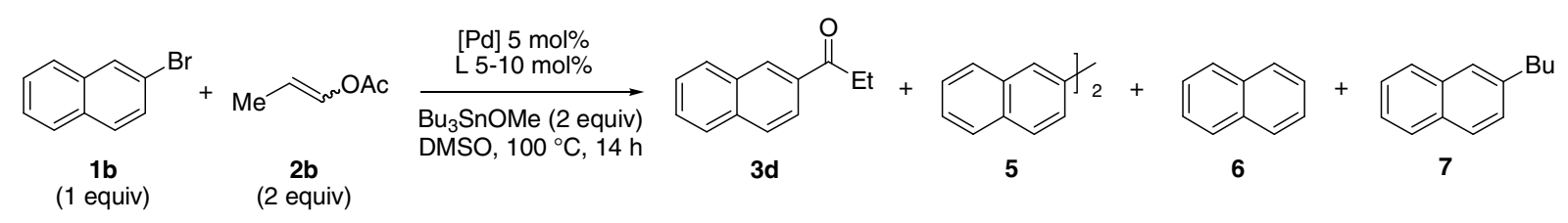

\begin{tabular}{cccccccc}
\hline \multirow{2}{*}{ Entry } & \multirow{2}{*}{ Catalyst } & \multirow{2}{*}{ Phosphine } & Conversion & \multicolumn{4}{c}{ Yields $(\%)$} \\
& & $(\%)$ & 3d & $\mathbf{5}$ & $\mathbf{6}$ & $\mathbf{7}$ \\
\hline 10 & $\mathrm{PdCl}_{2}\left[(o-\mathrm{Tol})_{3} \mathrm{P}\right]_{2}$ & - & 100 & 49 & 11 & 11 & 7 \\
\hline 11 & $\mathrm{PdCl}_{2}(\mathrm{MeCN})_{2}$ & $\mathrm{P}(o-\mathrm{Tol})_{3}$ & 68 & 32 & 9 & 3 & 3 \\
\hline 12 & $\mathrm{Pd}_{2}(\mathrm{dba})_{3}$ & $\mathrm{P}(o-\mathrm{Tol})_{3}$ & 100 & 56 & 6 & 6 & 8 \\
\hline 13 & $\mathrm{PdCl}_{2}\left(\mathrm{PPh}_{3}\right)_{2}$ & - & 100 & 16 & 21 & 7 & 8 \\
\hline 14 & $\mathrm{Pd}\left(\mathrm{PPh}_{3}\right)_{4}$ & - & 100 & 12 & 24 & 7 & 10 \\
\hline 15 & $\mathrm{PdCl}_{2}(\mathrm{dppf})$ & - & 100 & 43 & 8 & 8 & 7 \\
\hline 16 & $\mathrm{Pd}_{2}(\mathrm{dba})_{3}$ & $\mathrm{dppf}$ & 100 & 20 & 10 & 13 & 10 \\
\hline 17 & $\mathrm{Pd}_{2}(\mathrm{dba})_{3}$ & $\mathbf{L 1}$ & 100 & 3 & 20 & 8 & 6 \\
\hline 18 & $\mathrm{Pd}_{2}(\mathrm{dba})_{3}$ & $t$-Bu $\mathrm{P}$ & 100 & 3 & 28 & 3 & 3 \\
\hline 19 & $\mathrm{Pd}_{2}(\mathrm{dba})_{3}$ & $\mathbf{L 2}$ & 100 & 15 & 3 & 9 & 16 \\
\hline 20 & $\mathrm{Pd}_{2}(\mathrm{dba})_{3}$ & dppe & 92 & 27 & 16 & 7 & 9 \\
\hline 21 & $\mathrm{Pd}_{2}(\mathrm{dba})_{3}$ & dppp & 100 & 21 & 11 & 25 & 11 \\
\hline 22 & $\mathrm{Pd}_{2}(\mathrm{dba})_{3}$ & $r a c$-BINAP & 80 & 9 & 11 & 8 & 10 \\
\hline 23 & $\mathrm{Pd}_{2}(\mathrm{dba})_{3}$ & Xantphos & 100 & 18 & 3 & 7 & 9 \\
\hline
\end{tabular}




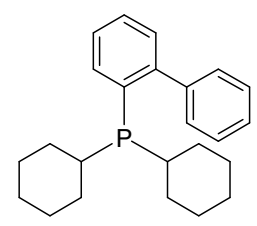

L1

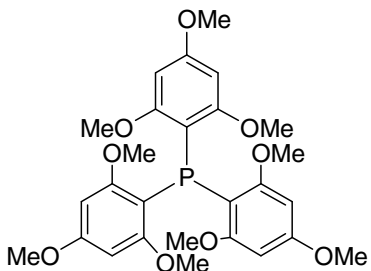

L2

The reaction was fairly sensitive to the nature of phosphines. The tri-o-tolylphosphine appeared as the best ligand. The nature of the palladium source seemed indifferent (similar results with $\mathrm{Pd}(0)$ and $\mathrm{Pd}(\mathrm{II}))$. For a more convenient work we decided to use the commercially available dichloro bis(tri-o-toylphosphine) palladium (II).

2.3 Study of the stoichiometry<smiles>Brc1ccc2ccccc2c1</smiles>

$1 \mathrm{~b}$ (1 equiv)
$R>\mathrm{OAC}$

2

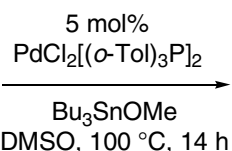

DMSO, $100^{\circ} \mathrm{C}, 14 \mathrm{~h}$

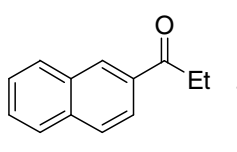

3d

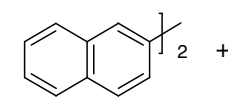

5<smiles>c1ccc2ccccc2c1</smiles>

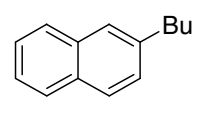

7

\begin{tabular}{cccccccc}
\hline \multirow{2}{*}{ Entry } & \multirow{2}{*}{ Vinylic acetate } & $\mathrm{Bu}_{3}$ SnOMe & $\begin{array}{c}\text { Conversion } \\
(\%)\end{array}$ & $\mathbf{3 d}$ & $\mathbf{5}$ & $\mathbf{6}$ & $\mathbf{7}$ \\
\hline 24 & $\mathbf{2 b}, 1,1$ equiv & 1,1 equiv & 86 & 26 & 5 & 17 & 16 \\
\hline 25 & $\mathbf{2 b}, 1,5$ equiv & 1,5 equiv & 99 & 42 & 7 & 7 & 17 \\
\hline 26 & $\mathbf{2 b}, 2,0$ equiv & 2,0 equiv & 100 & 49 & 11 & 11 & 7 \\
\hline 27 & $\mathbf{2 b}, 5,0$ equiv & 2,0 equiv & 100 & 55 & 3 & 8 & 4 \\
\hline 28 & $\mathbf{2 b}, 5,0$ equiv & 5,0 equiv & 100 & 65 & 4 & 1 & 8 \\
\hline 29 & $\mathbf{2 a}, 2,0$ equiv & 2,0 equiv & 100 & 41 & 2 & 6 & 3 \\
\hline 30 & $\mathbf{2 a}, 10,0$ equiv & 2,0 equiv & 100 & 51 & 1 & 1 & 2 \\
\hline
\end{tabular}

\subsection{Effect of the halogene source}

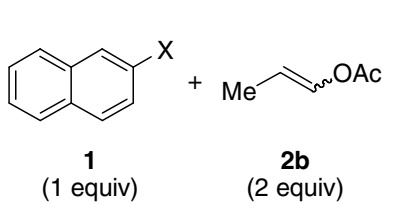

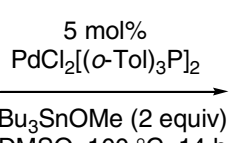

DMSO, $100^{\circ} \mathrm{C}, 14 \mathrm{~h}$

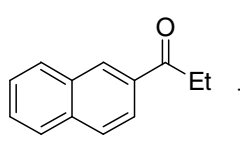

3d

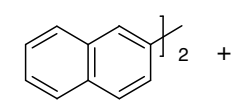

5

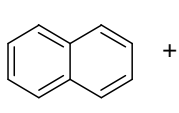

6<smiles>Brc1ccc2ccccc2c1</smiles>

7

\begin{tabular}{ccccccc}
\hline \multirow{2}{*}{ Entry } & \multirow{2}{*}{$\mathrm{X}$} & Conversion & \multicolumn{4}{c}{ Yields (\%) } \\
& & $(\%)$ & $\mathbf{3 d}$ & $\mathbf{5}$ & $\mathbf{6}$ & $\mathbf{7}$ \\
\hline 31 & $\mathbf{1 k}, \mathrm{Cl}$ & 33 & - & traces & - & - \\
\hline 33 & $\mathbf{1 b}, \mathrm{Br}$ & 100 & 49 & 11 & 11 & 7 \\
\hline 34 & $\mathbf{1 l}, \mathrm{I}$ & 100 & 30 & 10 & 31 & 11 \\
\hline 35 & $\mathbf{1 m}$, OTf & 57 & 17 & 10 & 2 & 4 \\
\hline
\end{tabular}




\section{Experimental procedures}

\section{6-Bromobenzothiazole ${ }^{1}(1 \mathrm{a})$}<smiles>Brc1ccc2ncsc2c1</smiles>

To a solution of 6-aminobenzothiazole $(1.0 \mathrm{~g}, 6.6 \mathrm{mmol})$ in water $(15.0 \mathrm{~mL})$ and hydrobromic acid $(15.0 \mathrm{~mL})$ was added, slowly, under stirring and cooling, a solution of sodium nitrite $(510 \mathrm{mg}, 7.3 \mathrm{mmol})$ in water $(1.0 \mathrm{~mL})$. The mixture was stirred under cooling $\left(0{ }^{\circ} \mathrm{C}\right)$ for half an hour and then added to a solution of copper(I) bromide $(1.1 \mathrm{~g}, 7.7 \mathrm{mmol})$ in hydrobromic acid $(15.0 \mathrm{~mL})$. The mixture was heated at $80{ }^{\circ} \mathrm{C}$ for 2 hours, then cooled to $0{ }^{\circ} \mathrm{C}$, alkalinised and extracted 4 times with diethyl ether. The product was purified by column chromatography on silica gel using $n$-Pentane/Et ${ }_{2} \mathrm{O} 8: 2$ as an eluent. White solid; $\mathrm{mp}=57-58{ }^{\circ} \mathrm{C}\left(55^{\circ} \mathrm{C}\right) ;^{1} 975$ $\mathrm{mg}, 69 \%$ yield; ${ }^{1} \mathrm{H}$ NMR $\left(270 \mathrm{MHz}, \mathrm{CDCl}_{3}\right):^{2} \delta 7.59(\mathrm{dd}, J=8.7 \mathrm{~Hz}, J=2.1 \mathrm{~Hz}, 1 \mathrm{H}), 7.97(\mathrm{~d}$, $J=8.7 \mathrm{~Hz}, 1 \mathrm{H}), 8.06(\mathrm{~d}, J=2.1 \mathrm{~Hz}, 1 \mathrm{H}), 8.95(\mathrm{~s}, 1 \mathrm{H}) ;{ }^{13} \mathrm{C} \mathrm{NMR}\left(67.5 \mathrm{MHz}, \mathrm{CDCl}_{3}\right):^{2} \delta$ 119.2, 124.3, 124.6, 129.6, 135.3, 152.0, 154.2; HRMS (EI): calcd for $\mathrm{C}_{7} \mathrm{H}_{4} \mathrm{NBrS}$ : 212.9248; found 212.9257 .

\section{2-Iodonaphthalene $\mathrm{e}^{3}(11)$}<smiles>Ic1ccc2ccccc2c1</smiles>

A mixture of 2-bromonaphthalene $(1.0 \mathrm{~g}, 4.8 \mathrm{mmol})$, potassium iodide $(12.0 \mathrm{~g}, 72.0 \mathrm{mmol})$, copper(I) iodide $(4.6 \mathrm{~g}, 24.0 \mathrm{mmol})$ and HMPA $(15.0 \mathrm{~mL})$ was stirred at $150-160{ }^{\circ} \mathrm{C}$ for 72 hours under nitrogen. The reaction was quenched by addition of dilute hydrochloric acid followed by diethyl ether and then filtered. The organic phase was separated, washed with sodium sulfite and water, dried $\left(\mathrm{Na}_{2} \mathrm{SO}_{4}\right)$ and evaporated. The product was purified by column chromatography on silica gel using $n$-Pentane as an eluent. Pale yellow solid; $\mathrm{mp}=$ $55-57{ }^{\circ} \mathrm{C}\left(53-54{ }^{\circ} \mathrm{C}\right) ;{ }^{4} 1.15 \mathrm{~g}, 94 \%$ yield; ${ }^{1} \mathrm{H}$ NMR $\left(270 \mathrm{MHz}, \mathrm{CDCl}_{3}\right):{ }^{5} \delta$ 7.41-7.52 (m, 3H), 7.63-7.77 (m, 3H), $8.19(\mathrm{~s}, 1 \mathrm{H}) ;{ }^{13} \mathrm{C} \mathrm{NMR}\left(67.5 \mathrm{MHz}, \mathrm{CDCl}_{3}\right):{ }^{5} \delta 91.5,126.4,126.6,126.7$, 127.8, 129.4, 132.0, 134.3, 134.9, 136.6; HRMS (EI): calcd for $\mathrm{C}_{10} \mathrm{H}_{7} \mathrm{I}$ : 253.9593; found 253.9582 .

\section{2-Naphthyl trifluoromethanesulfonate (1m)}<smiles>O=S(=O)(Oc1ccc2ccccc2c1)C(F)(F)F</smiles>

To naphth-2-ol $(1.0 \mathrm{~g}, 6.9 \mathrm{mmol})$ in anhydrous dichloromethane $(30.0 \mathrm{~mL})$ was added triethylamine $(1.4 \mathrm{~g}, 13.8 \mathrm{mmol})$ and the solution was cooled to $0{ }^{\circ} \mathrm{C}$. Trifluoromethanesulfonic anhydride $(2.3 \mathrm{~g}, 8.3 \mathrm{mmol})$ was added dropwise and the mixture was warmed up to room temperature. After 5 minutes, the mixture was diluted with diethyl ether, quenched with $10 \%$ aqueous hydrochloric acid and washed successively with saturated aqueous sodium bicarbonate solution and brine. After drying $\left(\mathrm{Na}_{2} \mathrm{SO}_{4}\right)$ the solvent was

\footnotetext{
${ }^{1}$ Boggust, W.; Cocker, W. J. Chem. Soc. 1949, 355-362.

${ }^{2}$ McKillop, A.; Sayer, T.; Bellinger, G. J. Org. Chem. 1976, 41, 1328-1331.

${ }^{3}$ Suzuki, H.; Kondo, A.; Ogawa, T. Chem. Lett. 1985, 411-412.

${ }^{4}$ Suzuki, H.; Kondo, A.; Inouye, M.; Ogawa, T. Synthesis 1986, 2, 121-122.

${ }^{5}$ Shen, H.-C.; Pal, S.; Lian, J.-J.; Liu, R.-S. J. Am. Chem. Soc. 2003, 125, 15762-15763.
} 
evaporated and the product was isolated by column chromatography on silica gel using $n$ Pentane as an eluent. White crystal; $\mathrm{mp}=30-31{ }^{\circ} \mathrm{C}\left(30-33{ }^{\circ} \mathrm{C}\right) ;{ }^{6} 1.77 \mathrm{~g}, 93 \%$ yield; ${ }^{1} \mathrm{H}$ NMR $\left(270 \mathrm{MHz}, \mathrm{CDCl}_{3}\right):^{6} \delta 7.36(\mathrm{dd}, J=9.0 \mathrm{~Hz}, J=2.6 \mathrm{~Hz}, 1 \mathrm{H}), 7.51-7.60(\mathrm{~m}, 2 \mathrm{H}), 7.74(\mathrm{~d}, J=$ $2.6 \mathrm{~Hz}, 1 \mathrm{H}), 7.83-7.91(\mathrm{~m}, 3 \mathrm{H}) ;{ }^{13} \mathrm{C}$ NMR $\left(67.5 \mathrm{MHz}, \mathrm{CDCl}_{3}\right): \delta 118.8(\mathrm{~d}, J=320.6 \mathrm{~Hz})$, 119.2, 119.5, 127.2, 127.6, 127.9, 128.0, 130.6, 132.3, 133.3, 147.1; HRMS (EI): calcd for $\mathrm{C}_{11} \mathrm{H}_{7} \mathrm{O}_{3} \mathrm{~F}_{3} \mathrm{~S}: 276.0068$; found 276.0060 .

Typical procedure for the preparation of vinylic acetates. To a solution of aldehyde (1.0 mmol $)$, acetic anhydride $(5.0 \mathrm{mmol})$ and triethylamine $(2.0 \mathrm{mmol})$ was added dimethylaminopyridine $(0.1 \mathrm{mmol})$. The solution was stirred at room temperature for 72 hours, then poured onto ice and the resulting mixture was stirred for an hour. The aqueous phase was separated and extracted twice with diethyl ether. The extract was washed with saturated aqueous sodium bicarbonate solution until neutral, then with water, brine, and dried $\left(\mathrm{Na}_{2} \mathrm{SO}_{4}\right)$. Diethyl ether was removed under vacuum and the product was purified by distillation or column chromatography on silica gel.

1-Propen-1-yl acetate $(2 b)$<smiles>CC=COC(C)=O</smiles>

Colorless oil; bp $=107{ }^{\circ} \mathrm{C}(760 \mathrm{mmHg}) ;{ }^{7} 45 \%$ yield $(Z: E=1: 2) ;{ }^{1} \mathrm{H}$ NMR $(270 \mathrm{MHz}$, $\mathrm{CDCl}_{3}$ ): $\delta 1.62-1.68(\mathrm{~m}, 6 \mathrm{H}$, isomers $Z$ and $E$ ), $2.10(\mathrm{~s}, 3 \mathrm{H}$, isomer $Z$ ), 2.16 (s, 3H, isomer $E$ ), 4.87-4.97 (qt, $J=6.9 \mathrm{~Hz}, 1 \mathrm{H}$, isomer $E$ ), $5.42(\mathrm{dq}, J=12.3 \mathrm{~Hz}, J=6.9 \mathrm{~Hz}, 1 \mathrm{H}$, isomer $Z$ ), 6.99-7.08 (m, 2H, isomers $Z$ and $E) ;{ }^{13} \mathrm{C}$ NMR $\left(67.5 \mathrm{MHz}, \mathrm{CDCl}_{3}\right): \delta 9.7,12.2,20.6,108.5$, 109.7, 136.0, 168.0; HRMS (EI): calcd for $\mathrm{C}_{5} \mathrm{H}_{8} \mathrm{O}_{2}$ : 100.0524; found 100.0526.

\section{2-Methyl-1-propen-1-yl acetate (2c)}<smiles>CC(=O)OC=C(C)C</smiles>

Colorless oil; bp $=124{ }^{\circ} \mathrm{C}(760 \mathrm{mmHg}) ;{ }^{7} 47 \%$ yield; ${ }^{1} \mathrm{H}$ NMR $\left(270 \mathrm{MHz}, \mathrm{CDCl}_{3}\right):{ }^{7} \delta 1.65(\mathrm{~d}$, $J=1.0 \mathrm{~Hz}, 3 \mathrm{H}), 1.68(\mathrm{~d}, J=1.3 \mathrm{~Hz}, 3 \mathrm{H}), 2.13(\mathrm{~s}, 3 \mathrm{H}), 6.83-6.85(\mathrm{~m}, 1 \mathrm{H}) ;{ }^{13} \mathrm{C} \mathrm{NMR}(67.5$ $\mathrm{MHz}, \mathrm{CDCl}_{3}$ ): $\delta 15.5,19.5,20.6,118.1,129.9,168.24$; HRMS (EI): calcd for $\mathrm{C}_{6} \mathrm{H}_{10} \mathrm{O}_{2}$ : 114.0681; found 114.0688.

\section{3-Phenyl-1-propen-1-yl acetate (2d)}<smiles>CC(=O)O/C=C/Cc1ccccc1</smiles>

Colorless oil; eluent, $\mathrm{CH}_{2} \mathrm{Cl}_{2} / n$-Pentane $=1 / 1 ; 59 \%$ yield $(Z: E=1: 1) ;{ }^{1} \mathrm{H}$ NMR $(270 \mathrm{MHz}$, $\left.\mathrm{CDCl}_{3}\right): \delta 2.10(\mathrm{~s}, 3 \mathrm{H}$, isomer $Z), 2.16(\mathrm{~s}, 3 \mathrm{H}$, isomer $E), 3.33(\mathrm{~d}, J=7.7 \mathrm{~Hz}, 2 \mathrm{H}$, isomer $Z$ ), $3.50(\mathrm{~d}, J=7.4 \mathrm{~Hz}, 2 \mathrm{H}$, isomer $E), 5.08(\mathrm{dt}, J=7.4 \mathrm{~Hz}, J=6.4 \mathrm{~Hz}, 1 \mathrm{H}$, isomer $E), 5.58(\mathrm{dt}, J$ $=12.6 \mathrm{~Hz}, J=7.7 \mathrm{~Hz}, 1 \mathrm{H}$, isomer $Z), 7.13-7.31(\mathrm{~m}, 12 \mathrm{H}$, isomers $Z$ and $E) ;{ }^{13} \mathrm{C} \mathrm{NMR}(67.5$

\footnotetext{
${ }^{6}$ Brimble, M. A.; Lai, M. Y. H. Org. Biomol. Chem. 2003, 1, 2084-2095.

${ }^{7}$ Barbier, P.; Benezra, C. J. Org. Chem. 1983, 48, 2705-2709.
} 
$\left.\mathrm{MHz}, \mathrm{CDCl}_{3}\right): \delta 20.6,20.7,30.6,33.5,112.4,113.7,126.1,126.3,128.2,128.3,128.5,134.6$, 136.3, 139.7, 140.0, 168.1; HRMS (EI): calcd for $\mathrm{C}_{11} \mathrm{H}_{12} \mathrm{O}_{2}$ : 176.0837; found 176.0831.

\section{3-Phenyl-1-propen-1-yl-1d $d_{1}$ acetate (2e)}

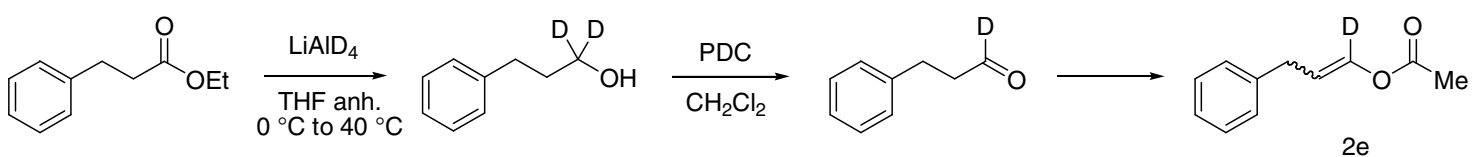

3-Phenylpropan-1-ol-1 $d_{2}$<smiles>[2H]C([2H])(O)CCc1ccccc1</smiles>

To a cold solution $\left(0{ }^{\circ} \mathrm{C}\right)$ of ethyl 3-phenylpropanoate $(4.2 \mathrm{~g}, 23.8 \mathrm{mmol})$ in anhydrous tetrahydrofurane $(20.0 \mathrm{~mL})$ was added lithium aluminium deuteride $(1.0 \mathrm{~g}, 23.8 \mathrm{mmol})$ by portion. After one hour, the suspension was heated at $40{ }^{\circ} \mathrm{C}$ for 2 hours then cooled to room temperature. Water was added to destroy excess of lithium aluminium deuteride and, after filtration, the mixture was extracted 3 times with diethyl ether. The organic phase was washed with water and brine then dried $\left(\mathrm{Na}_{2} \mathrm{SO}_{4}\right)$. The solvent was removed under vacuum to obtain a colorless oil used without purification. Colorless oil; $3.29 \mathrm{~g}$, quantitative yield; ${ }^{1} \mathrm{H}$ NMR (270 $\left.\mathrm{MHz}, \mathrm{CDCl}_{3}\right):^{8} \delta 1.45(\mathrm{~s}, 1 \mathrm{H}), 1.88(\mathrm{t}, J=7.7 \mathrm{~Hz}, 2 \mathrm{H}), 2.70(\mathrm{t}, J=7.7 \mathrm{~Hz}, 2 \mathrm{H}), 7.16-7.31(\mathrm{~m}$, $5 \mathrm{H}) ;{ }^{13} \mathrm{C} \mathrm{NMR}\left(67.5 \mathrm{MHz}, \mathrm{CDCl}_{3}\right)^{8}: \delta 32.2,34.1,61.7(\mathrm{t}, J=21.8 \mathrm{~Hz}), 126.0,128.5,141.9$; HRMS (EI): calcd for $\mathrm{C}_{9} \mathrm{H}_{10} \mathrm{D}_{2} \mathrm{O}$ : 138.1014; found 138.1018.

3-Phenylpropanal-1 $d_{1}$<smiles>[2H]C(=O)CCc1ccccc1</smiles>

To a solution of 3-phenylpropan-1-ol-1 $d_{2}(3.8 \mathrm{~g}, 27.5 \mathrm{mmol})$ in dichloromethane $(100.0 \mathrm{~mL})$ was added pyridinium dichromate $(20.3 \mathrm{~g}, 55.0 \mathrm{mmol})$. The suspension was stirred at room temperature for 20 hours then filtered. The solvent was removed under vacuum and the product isolated by column chromatography on silica gel using dichloromethane as eluent. Colorless oil; $2.53 \mathrm{~g}, 68 \%$ yield; ${ }^{1} \mathrm{H}$ NMR $\left(270 \mathrm{MHz}, \mathrm{CDCl}_{3}\right): \delta 2.73-2.79(\mathrm{~m}, 2 \mathrm{H}), 2.95(\mathrm{t}, J$ $=7.4 \mathrm{~Hz}, 2 \mathrm{H}), 7.17-7.32(\mathrm{~m}, 5 \mathrm{H}) ;{ }^{13} \mathrm{C} \mathrm{NMR}\left(67.5 \mathrm{MHz}, \mathrm{CDCl}_{3}\right): \delta 28.1,45.0(\mathrm{t}, J=5.6 \mathrm{~Hz})$, 126.3, 128.2, 128.4, 128.6, 140.3, 201.2 (t, $J=26.6 \mathrm{~Hz}$ ); HRMS (EI): calcd for $\mathrm{C}_{9} \mathrm{H}_{9} \mathrm{DO}$ : 135.0794; found 135.0790.

3-Phenyl-1-propen-1-yl-1 $d_{l}$ acetate (2e)<smiles>[2H]/C(=C\Cc1ccccc1)OC(C)=O</smiles>

Colorless oil; eluent, $\mathrm{CH}_{2} \mathrm{Cl}_{2} / n$-Pentane $=1 / 1 ; 51 \%$ yield $(Z: E=55: 45) ;{ }^{1} \mathrm{H}$ NMR $(270 \mathrm{MHz}$, $\left.\mathrm{CDCl}_{3}\right): \delta 2.11(\mathrm{~s}, 3 \mathrm{H}$, isomer $Z), 2.17(\mathrm{~s}, 3 \mathrm{H}$, isomer $E), 3.33(\mathrm{~d}, J=7.7 \mathrm{~Hz}, 2 \mathrm{H}$, isomer $Z$ ), $3.50(\mathrm{~d}, J=7.4 \mathrm{~Hz}, 2 \mathrm{H}$, isomer $E), 5.08(\mathrm{t}, J=7.4 \mathrm{~Hz}, 1 \mathrm{H}$, isomer $E), 5.57(\mathrm{tt}, J=7.7 \mathrm{~Hz}, J=$

\footnotetext{
${ }^{8}$ McGrath, M. J.; O’Brien, P. Synthesis 2006, 13, 2233-2241.
} 
$1.7 \mathrm{~Hz}, 1 \mathrm{H}$, isomer $Z), 7.18-7.32(\mathrm{~m}, 10 \mathrm{H}$, isomers $Z$ and $E) ;{ }^{13} \mathrm{C} \mathrm{NMR}\left(67.5 \mathrm{MHz}, \mathrm{CDCl}_{3}\right): \delta$ 20.6, 20.7, 30.6, 33.5, 112.2, 113.6, 126.1, 128.2, 128.3, 128.5, 134.4 (t, $J=29.6 \mathrm{~Hz}$ ), 136.1 (t, $J=29.0 \mathrm{~Hz}$ ), 139.7, 140.0, 168.0, 168.1; HRMS (EI): calcd for $\mathrm{C}_{11} \mathrm{H}_{11} \mathrm{DO}_{2}: 177.0900$; found 177.0910.

Typical procedure for the synthesis of aryl ketones. To a solution of aryl bromide (1.0 mmol) in anhydrous dimethylsulfoxide $(0.5 \mathrm{~mL} / \mathrm{mmol})$ was added enol acetate $(2.0 \mathrm{mmol})$, tributyltin methoxide $(2.0 \mathrm{mmol})$ followed by the catalyst $(5 \mathrm{~mol} \%)$. The mixture was heated under nitrogen at $100{ }^{\circ} \mathrm{C}$ for 14 hours then cooled to room temperature. The reaction mixture was diluted with ethyl acetate and a solution of aqueous potassium fluoride $4 \mathrm{M}$ was added. The mixture was stirred vigorously for 1 hour and then filtered through celite. The organic phase was washed twice with water then dried $\left(\mathrm{Na}_{2} \mathrm{SO}_{4}\right)$. After filtration, ethyl acetate was removed under vacuum and the product was purified by column chromatography on silica gel or preparative TLC.

\section{6-Acetylbenzothiazole (3a)}<smiles>CC(=O)c1ccc2ncsc2c1</smiles>

White solid; $\mathrm{mp}=96-97{ }^{\circ} \mathrm{C}\left(94{ }^{\circ} \mathrm{C}\right) ;{ }^{9}$ eluent, $\mathrm{CH}_{2} \mathrm{Cl}_{2} ; 49 \%$ yield; ${ }^{1} \mathrm{H}$ NMR $(270 \mathrm{MHz}$, $\left.\mathrm{CDCl}_{3}\right):{ }^{9} \delta 2.71(\mathrm{~s}, 3 \mathrm{H}), 8.12(\mathrm{dd}, J=8.6 \mathrm{~Hz}, J=1.6 \mathrm{~Hz}, 1 \mathrm{H}), 8.19(\mathrm{~d}, J=8.7 \mathrm{~Hz}, 1 \mathrm{H}), 8.61$ $(\mathrm{d}, J=1.6 \mathrm{~Hz}, 1 \mathrm{H}), 9.17(\mathrm{~s}, 1 \mathrm{H}) ;{ }^{13} \mathrm{C}$ NMR $\left(67.5 \mathrm{MHz}, \mathrm{CDCl}_{3}\right): \delta 26.9,122.9,123.6,126.2$, 134.1, 134.4, 156.0, 157.6, 197.0; HRMS (EI): calcd for $\mathrm{C}_{9} \mathrm{H}_{7} \mathrm{NOS}$ : 177.0248; found 177.0252 .

\section{(Benzothiazol-6-yl) propan-1-one (3b)}<smiles>CCC(=O)c1ccc2ncsc2c1</smiles>

White solid; $\mathrm{mp}=118-119{ }^{\circ} \mathrm{C}$; eluent, $\mathrm{CH}_{2} \mathrm{Cl}_{2} ; 56 \%$ yield; ${ }^{1} \mathrm{H}$ NMR $\left(270 \mathrm{MHz}, \mathrm{CDCl}_{3}\right): \delta$ $1.28(\mathrm{t}, J=7.2 \mathrm{~Hz}, 3 \mathrm{H}), 3.11(\mathrm{q}, J=7.2 \mathrm{~Hz}, 2 \mathrm{H}), 8.11-8.21(\mathrm{~m}, 2 \mathrm{H}), 8.62(\mathrm{~d}, J=1.0 \mathrm{~Hz}, 1 \mathrm{H})$, $9.16(\mathrm{~s}, 1 \mathrm{H}) ;{ }^{13} \mathrm{C}$ NMR $\left(67.5 \mathrm{MHz}, \mathrm{CDCl}_{3}\right): \delta 8.3,32.1,122.5,123.6,125.9,134.1,134.2$, 155.9, 157.3, 199.7; HRMS (EI): calcd for $\mathrm{C}_{10} \mathrm{H}_{9} \mathrm{NOS}$ : 191.0405; found 191.0409.

\section{2-Acetylnaphthalene (3c)}<smiles>CC(=O)c1ccc2ccccc2c1</smiles>

White solid; $\mathrm{mp}=55-56{ }^{\circ} \mathrm{C}\left(54-56{ }^{\circ} \mathrm{C}\right) ;{ }^{10}$ eluent, $\mathrm{CH}_{2} \mathrm{Cl}_{2} / n$-Pentane $=8 / 2 ; 41 \%$ yield; ${ }^{1} \mathrm{H}$ NMR (270 MHz, $\left.\mathrm{CDCl}_{3}\right):{ }^{11} \delta 2.73(\mathrm{~s}, 3 \mathrm{H}), 7.53-7.64(\mathrm{~m}, 2 \mathrm{H}), 7.87-8.06(\mathrm{~m}, 4 \mathrm{H}), 8.47$ (s,

\footnotetext{
${ }^{9}$ Al Hariri, M.; Jouve, K.; Pautet, F.; Domard, M.; Fenet, B.; Fillion, H. J. Org. Chem. 1997, 62, 405-410.

${ }^{10}$ Giurg, M.; Brzaszcz, M.; Mlochowski, J. Pol. J. Chem. 2006, 80, 417-428.

${ }^{11}$ Hansen, A. L.; Skrydstrup, T. J. Org. Chem. 2005, 70, 5997-6003.
} 
$1 \mathrm{H}) ;{ }^{13} \mathrm{C}$ NMR $\left(67.5 \mathrm{MHz}, \mathrm{CDCl}_{3}\right):{ }^{11} \delta 26.6,123.9,126.8,127.8,128.4,128.5,129.5,130.2$, 132.5, 134.5, 135.6, 198.1; HRMS (EI): calcd for $\mathrm{C}_{12} \mathrm{H}_{10} \mathrm{O}$ : 170.0732 ; found 170.0737.

\section{1-(Naphthalen-2-yl) propan-1-one (3d)}<smiles>CCC(=O)c1ccc2ccccc2c1</smiles>

White solid; $\mathrm{mp}=64-65{ }^{\circ} \mathrm{C}\left(60-61{ }^{\circ} \mathrm{C}\right) ;{ }^{12}$ eluent, $\mathrm{CH}_{2} \mathrm{Cl}_{2} / n$-Pentane $=8 / 2 ; 49 \%$ yield; ${ }^{1} \mathrm{H}$ NMR (270 MHz, $\left.\mathrm{CDCl}_{3}\right):{ }^{12} \delta 1.28(\mathrm{t}, J=7.3 \mathrm{~Hz}, 3 \mathrm{H}), 3.11(\mathrm{q}, J=7.3 \mathrm{~Hz}, 2 \mathrm{H}), 7.49-7.61(\mathrm{~m}$, 2H), 7.84-8.04 (m, 4H), $8.45(\mathrm{~s}, 1 \mathrm{H}) ;{ }^{13} \mathrm{C}$ NMR $\left(67.5 \mathrm{MHz}, \mathrm{CDCl}_{3}\right): \delta 8.3,31.8,123.9,126.6$, 127.7, 128.2, 128.3, 129.4, 129.5, 132.5, 134.2, 135.5, 200.7; HRMS (EI): calcd for $\mathrm{C}_{13} \mathrm{H}_{12} \mathrm{O}$ : 184.0888; found 184.0891 .

\section{2-Methyl-1-(naphthalen-2-yl) propan-1-one (3e)}<smiles>CC(C)C(=O)c1ccc2ccccc2c1</smiles>

Colorless oil; eluent, $\mathrm{CH}_{2} \mathrm{Cl}_{2} / n$-Pentane $=7 / 3 ; 75 \%$ yield; ${ }^{1} \mathrm{H}$ NMR $\left(270 \mathrm{MHz}, \mathrm{CDCl}_{3}\right):{ }^{13} \delta$ $1.28(\mathrm{~d}, J=6.8 \mathrm{~Hz}, 6 \mathrm{H}), 3.73$ (heptuplet, $J=6.8 \mathrm{~Hz}, 1 \mathrm{H}), 7.51-7.62(\mathrm{~m}, 2 \mathrm{H}), 7.86-8.05$ (m, $4 \mathrm{H}), 8.47(\mathrm{~s}, 1 \mathrm{H}) ;{ }^{13} \mathrm{C}$ NMR $\left(67.5 \mathrm{MHz}, \mathrm{CDCl}_{3}\right):{ }^{13} \delta$ 19.3, 35.4, 124.3, 126.7, 127.7, 128.3, 128.4, 129.5, 129.6, 132.6, 133.5, 135.5, 204.4; HRMS (EI): calcd for $\mathrm{C}_{14} \mathrm{H}_{14} \mathrm{O}$ : 198.1045; found 198.1047.

\section{1-(Naphthalen-2-yl)-3-phenylpropan-1-one (3f)}<smiles>O=C(CCc1ccccc1)c1ccc2ccccc2c1</smiles>

White solid; $\mathrm{mp}=90-91{ }^{\circ} \mathrm{C}\left(85^{\circ} \mathrm{C}\right) \dot{1}^{14}$ eluent, $\mathrm{CH}_{2} \mathrm{Cl}_{2} / n$-Pentane $=7 / 3 ; 72 \%$ yield; ${ }^{1} \mathrm{H}$ NMR $\left(270 \mathrm{MHz}, \mathrm{CDCl}_{3}\right):^{14} \delta 3.12(\mathrm{t}, J=7.7 \mathrm{~Hz}, 2 \mathrm{H}), 3.43(\mathrm{t}, J=7.7 \mathrm{~Hz}, 2 \mathrm{H}), 7.18-7.34(\mathrm{~m}, 5 \mathrm{H})$, $7.50-7.61(\mathrm{~m}, 2 \mathrm{H}), 7.84-7.93(\mathrm{~m}, 3 \mathrm{H}), 8.03(\mathrm{dd}, J=8.8 \mathrm{~Hz}, J=1.7 \mathrm{~Hz}, 1 \mathrm{H}), 8.45(\mathrm{~s}, 1 \mathrm{H}) ;{ }^{13} \mathrm{C}$ NMR $\left(67.5 \mathrm{MHz}, \mathrm{CDCl}_{3}\right):^{14} \delta 30.2,40.5,123.8,126.1,126.7,127.7,128.4,128.5,129.5$, 129.6, 132.5, 134.2, 135.6, 141.3, 199.1; HRMS (EI): calcd for $\mathrm{C}_{19} \mathrm{H}_{16} \mathrm{O}$ : 260.1201; found 260.1207 .

\footnotetext{
${ }^{12}$ Azzolina, O.; Collina, S.; Urbano, M.; Fata, E.; Loddo, G.; Linati, L.; Lanza, E.; Barbieri, A. Chirality 2006, 18, 841-848.

${ }^{13}$ Lee, S. W.; Lee, K.; Seomoon, D.; Kim, S.; Kim, H.; Kim, H.; Shim, E.; Lee, S.; Kim, M.; Lee, P. H. J. Org. Chem. 2004, 69, 4852-4855.

${ }^{14}$ Martinez, R.; Ramon, D. J.; Yus, M. Tetrahedron 2006, 62, 8988-9001.
} 
<smiles>CC(C)C(=O)c1cccc2ccccc12</smiles>

Colorless oil; eluent, $\mathrm{CH}_{2} \mathrm{Cl}_{2} / n$-Pentane $=7 / 3 ; 76 \%$ yield; ${ }^{1} \mathrm{H}$ NMR $\left(270 \mathrm{MHz}, \mathrm{CDCl}_{3}\right): \delta 1.24$ $(\mathrm{d}, J=6.9 \mathrm{~Hz}, 6 \mathrm{H}), 3.51$ (heptuplet, $J=6.9 \mathrm{~Hz}, 1 \mathrm{H}), 7.45-7.59(\mathrm{~m}, 3 \mathrm{H}), 7.73(\mathrm{dd}, J=7.2 \mathrm{~Hz}$, $J=1.3 \mathrm{~Hz}, 1 \mathrm{H}), 7.85-7.96(\mathrm{~m}, 2 \mathrm{H}), 8.28-8.31(\mathrm{~m}, 1 \mathrm{H}) ;{ }^{13} \mathrm{C} \mathrm{NMR}\left(67.5 \mathrm{MHz}, \mathrm{CDCl}_{3}\right): \delta 18.7$, 39.6, 124.5, 125.6, 125.8, 126.4, 127.5, 128.3, 130.5, 131.6, 133.9, 137.0, 209.0; HRMS (EI): calcd for $\mathrm{C}_{14} \mathrm{H}_{14} \mathrm{O}$ : 198.10445 ; found 198.1047

\section{1-(4-Nitrophenyl) propan-1-one (3h)}<smiles>CCC(=O)c1ccc([N+](=O)[O-])cc1</smiles>

Yellow solid; $\mathrm{mp}=86-88{ }^{\circ} \mathrm{C}\left(92{ }^{\circ} \mathrm{C}\right){ }^{15}$ eluent, Hexane/AcOEt $=9 / 1 ; 12 \%$ yield; ${ }^{1} \mathrm{H}$ NMR $\left(270 \mathrm{MHz} \mathrm{CDCl}_{3}\right):^{16} \delta 1.26(\mathrm{t}, J=7.2 \mathrm{~Hz}, 3 \mathrm{H}), 3.06(\mathrm{q}, J=7.2 \mathrm{~Hz}, 2 \mathrm{H}), 8.12(\mathrm{dt}, J=9.1 \mathrm{~Hz}$, $J=2.1 \mathrm{~Hz}, 2 \mathrm{H}), 8.32(\mathrm{dt}, J=9.1 \mathrm{~Hz}, J=2.1 \mathrm{~Hz}, 2 \mathrm{H}) ;{ }^{13} \mathrm{C} \mathrm{NMR}\left(67.5 \mathrm{MHz}, \mathrm{CDCl}_{3}\right): \delta 7.9$, 32.4, 123.9, 129.0, 141.4, 199.0; HRMS (EI): calcd for $\mathrm{C}_{9} \mathrm{H}_{9} \mathrm{NO}_{3}$ : 179.0582; found 179.0586.

\section{1-(4-Methoxyphenyl) propan-1-one (3i)}<smiles>CCC(=O)c1ccc(OC)cc1</smiles>

Colorless oil; eluent, $\mathrm{CH}_{2} \mathrm{Cl}_{2} / n$-Pentane $=8 / 2 ; 47 \%$ yield; ${ }^{1} \mathrm{H}$ NMR $\left(270 \mathrm{MHz}, \mathrm{CDCl}_{3}\right):{ }^{17} \delta$ $1.21(\mathrm{t}, J=7.3 \mathrm{~Hz}, 3 \mathrm{H}), 2.95(\mathrm{q}, J=7.3 \mathrm{~Hz}, 2 \mathrm{H}), 3.87(\mathrm{~s}, 3 \mathrm{H}), 6.93(\mathrm{dt}, J=9.4 \mathrm{~Hz}, J=2.5$ $\mathrm{Hz}, 2 \mathrm{H}), 7.95(\mathrm{dt}, J=9.4 \mathrm{~Hz}, J=2.5 \mathrm{~Hz}, 2 \mathrm{H}) ;{ }^{13} \mathrm{C} \mathrm{NMR}\left(67.5 \mathrm{MHz}, \mathrm{CDCl}_{3}\right){ }^{17} \delta 8.4,31.4$, 55.4, 113.7, 114.2, 130.2, 163.3, 199.4; HRMS (EI): calcd for $\mathrm{C}_{10} \mathrm{H}_{12} \mathrm{O}_{2}$ : 164.0837; found 164.0843

1-(4-Methoxyphenyl)-2-methylpropan-1-one (3j)<smiles>COc1ccc(C(=O)C(C)C)cc1</smiles>

\footnotetext{
${ }^{15}$ Zimmer, H.; Bercz, J. P. Justus Liebigs Ann. Chem. 1965, 686, 107-114.

${ }^{16}$ Dai, Y.; Guo, Y.; Frey, R. R.; Ji, Z.; Curtin, M. L.; Ahmed, A. A.; Albert, D. H.; Arnold, L.; Arries, S.; Barlozzari, T.; Bauch, J. L.; Bouska, J. J.; Bousquet, P. F.; Cunha, G. A.; Glaser, K. B.; Guo, J.; Li, J.; Marcotte, P. A.; Marsh, K. C.; Moskey, M. D.; Pease, L. J.; Stewart, K. D.; Stoll, V. S.; Tapang, P.; Wishart, N.; Davidsen, S. K.; Michaelides, M. R. J. Med. Chem. 2005, 48, 6066-6083.

${ }^{17}$ Yamane, M.; Kubota, Y.; Narasaka, K. Bull. Chem. Soc. Jpn 2005, 78, 331-340.
} 
Colorless oil; eluent, $\mathrm{CH}_{2} \mathrm{Cl}_{2} / n$-Pentane $=8 / 2 ; 58 \%$ yield; ${ }^{1} \mathrm{H}$ NMR $\left(270 \mathrm{MHz}, \mathrm{CDCl}_{3}\right): \delta 1.21$ $(\mathrm{d}, J=6.9 \mathrm{~Hz}, 6 \mathrm{H}), 3.52$ (heptuplet, $J=6.9 \mathrm{~Hz}, 1 \mathrm{H}), 3.87(\mathrm{~s}, 3 \mathrm{H}), 6.94(\mathrm{dt}, J=9.5 \mathrm{~Hz}, J=2.4$ $\mathrm{Hz}, 2 \mathrm{H}), 7.95(\mathrm{dt}, J=9.5 \mathrm{~Hz}, J=2.4 \mathrm{~Hz}, 2 \mathrm{H}) ;{ }^{13} \mathrm{C} \mathrm{NMR}\left(67.5 \mathrm{MHz}, \mathrm{CDCl}_{3}\right): \delta 19.3,34.9$, 55.4, 113.7, 129.2, 130.5, 163.3, 203.1; HRMS (EI): calcd for $\mathrm{C}_{11} \mathrm{H}_{14} \mathrm{O}_{2}$ : 178.0994; found 178.1000

1-[4-(Dimethylamino) phenyl]-3-phenylpropan-1-one (3k)<smiles>CN(C)c1ccc(C(=O)CCc2ccccc2)cc1</smiles>

Yellow solid; $\mathrm{mp}=86-88{ }^{\circ} \mathrm{C}\left(88-89{ }^{\circ} \mathrm{C}\right) ;{ }^{18}$ eluent, $\mathrm{CH}_{2} \mathrm{Cl}_{2} / n$-Pentane $=8 / 2 ; 69 \%$ yield; ${ }^{1} \mathrm{H}$ NMR (270 MHz, CDCl 3$):^{18} \delta 3.01-3.09(\mathrm{~m}, 8 \mathrm{H}), 3.17-3.23(\mathrm{~m}, 2 \mathrm{H}), 6.63(\mathrm{dt}, J=9.6 \mathrm{~Hz}, J=$ $2.5 \mathrm{~Hz}, 2 \mathrm{H}), 7.16-7.32(\mathrm{~m}, 5 \mathrm{H}), 7.88(\mathrm{dt}, J=9.6 \mathrm{~Hz}, J=2.5 \mathrm{~Hz}, 2 \mathrm{H}) ;{ }^{13} \mathrm{C}$ NMR $(67.5 \mathrm{MHz}$, $\left.\mathrm{CDCl}_{3}\right):{ }^{18} \delta 30.7,39.7,40.0,110.6,124.9,125.9,128.4,130.2,141.8,153.4,197.3$; HRMS (EI): calcd for $\mathrm{C}_{17} \mathrm{H}_{19} \mathrm{NO}$ : 253.1467; found 253.1473.

\section{1-[2-(Dimethylamino) phenyl]-3-phenylpropan-1-one (3I)}<smiles>CN(C)c1ccccc1C(=O)CCc1ccccc1</smiles>

Yellow oil; eluent, $\mathrm{CH}_{2} \mathrm{Cl}_{2} / n$-Pentane $=8 / 2 ; 75 \%$ yield; ${ }^{1} \mathrm{H}$ NMR $\left(270 \mathrm{MHz}, \mathrm{CDCl}_{3}\right): \delta 2.70$ (s, 6H), $2.99(\mathrm{t}, J=7.7 \mathrm{~Hz}, 2 \mathrm{H}), 3.31(\mathrm{t}, J=7.7 \mathrm{~Hz}, 2 \mathrm{H}), 6.89-7.00(\mathrm{~m}, 2 \mathrm{H}), 7.14-7.37(\mathrm{~m}$, $7 \mathrm{H}) ;{ }^{13} \mathrm{C}$ NMR $\left(67.5 \mathrm{MHz}, \mathrm{CDCl}_{3}\right): \delta 30.9,42.9,44.5,117.2,120.6,125.9,128.3,128.5$, 129.2, 131.5, 133.0, 141.4, 151.7, 205.9; HRMS (EI): calcd for $\mathrm{C}_{17} \mathrm{H}_{19} \mathrm{NO}$ : 253.1467; found 253.1473

\section{1-(2-Methylphenyl)-3-phenylpropan-1-one (3m)}<smiles>Cc1ccccc1C(=O)CCc1ccccc1</smiles>

Colorless oil; eluent, $\mathrm{CH}_{2} \mathrm{Cl}_{2} / n$-Pentane $=7 / 3 ; 60 \%$ yield; ${ }^{1} \mathrm{H}$ NMR $\left(270 \mathrm{MHz}, \mathrm{CDCl}_{3}\right):{ }^{19} \delta$ $2.47(\mathrm{~s}, 3 \mathrm{H}), 3.04(\mathrm{t}, J=7.4 \mathrm{~Hz}, 2 \mathrm{H}), 3.20-3.26(\mathrm{~m}, 2 \mathrm{H}), 7.19-7.236(\mathrm{~m}, 8 \mathrm{H}), 7.58-7.61(\mathrm{~m}$, $1 \mathrm{H}) ;{ }^{13} \mathrm{C}$ NMR $\left(67.5 \mathrm{MHz}, \mathrm{CDCl}_{3}\right): \delta 21.2,30.3,43.2,125.6,126.1,128.3,128.4,128.5$, 129.4, 131.2, 131.9, 138.0, 141.2, 203.4; HRMS (EI): calcd for $\mathrm{C}_{16} \mathrm{H}_{16} \mathrm{O}$ : 224.1201; found 224.1208 .

\footnotetext{
${ }^{18}$ Tatamidani, H.; Yokota, K.; Kakiuchi, F.; Chatani, N. J. Org. Chem. 2004, 69, 5615-5621.

${ }^{19}$ Nonoshita, K.; Maruoka, K.; Yamamoto, H. Bull. Chem. Soc. Jpn 1988, 61, 2241-2243.
} 
<smiles>Cc1cccc(C)c1C(=O)CCc1ccccc1</smiles>

Colorless oil; eluent, $\mathrm{CH}_{2} \mathrm{Cl}_{2} / n$-Pentane $=7 / 3 ; 40 \%$ yield; ${ }^{1} \mathrm{H}$ NMR $\left(270 \mathrm{MHz}, \mathrm{CDCl}_{3}\right): \delta 2.15$ $(\mathrm{s}, 6 \mathrm{H}), 3.02-3.06(\mathrm{~m}, 4 \mathrm{H}), 6.99(\mathrm{~d}, J=7.4 \mathrm{~Hz}, 2 \mathrm{H}), 7.11-7.32(\mathrm{~m}, 6 \mathrm{H}) ;{ }^{13} \mathrm{C}$ NMR $(67.5 \mathrm{MHz}$, $\mathrm{CDCl}_{3}$ ): $\delta 19.01,29.4,46.2,126.1,127.7,128.5,132.5,140.9,142.2,209.4$; HRMS (EI): calcd for $\mathrm{C}_{17} \mathrm{H}_{18} \mathrm{O}$ : 238.1358; found 238.1348

\section{3-Phenyl-1-(pyridin-3-yl) propan-1-one (3o)}<smiles>O=C(CCc1ccccc1)c1cccnc1</smiles>

Colorless oil; eluent, $\mathrm{CH}_{2} \mathrm{Cl}_{2} / \mathrm{AcOEt}=9 / 1 ; 30 \%$ yield; ${ }^{1} \mathrm{H} \mathrm{NMR}\left(270 \mathrm{MHz}, \mathrm{CDCl}_{3}\right): \delta 3.09(\mathrm{t}$, $J=7.6 \mathrm{~Hz}, 2 \mathrm{H}), 3.32(\mathrm{t}, J=7.6 \mathrm{~Hz}, 2 \mathrm{H}), 7.21-7.34(\mathrm{~m}, 5 \mathrm{H}), 7.40(\mathrm{ddd}, J=7.9 \mathrm{~Hz}, J=4.9 \mathrm{~Hz}$, $J=0.8 \mathrm{~Hz}, 1 \mathrm{H}), 8.22(\mathrm{dt}, J=7.9 \mathrm{~Hz}, J=1.8 \mathrm{~Hz}, 1 \mathrm{H}), 8.77(\mathrm{dd}, J=4.9 \mathrm{~Hz}, J=1.8 \mathrm{~Hz}, 1 \mathrm{H})$, $7.16(\mathrm{~d}, J=1.8 \mathrm{~Hz}, 1 \mathrm{H}) ;{ }^{13} \mathrm{C} \mathrm{NMR}\left(67.5 \mathrm{MHz}, \mathrm{CDCl}_{3}\right): \delta 29.8,40.6,123.6,126.3,128.4$, 128.6, 135.3, 140.7, 149.6, 150.1, 153.5, 198.0; HRMS (EI): calcd for $\mathrm{C}_{14} \mathrm{H}_{13} \mathrm{NO}$ : 211.0997; found 211.0990 .

\section{1-(Naphthalen-2-yl)-3-phenylpropan-1-one- $2 d_{1}(3 p)$}<smiles>[2H]C(Cc1ccccc1)C(=O)c1ccc2ccccc2c1</smiles>

White solid; $\mathrm{mp}=91-92{ }^{\circ} \mathrm{C}$; eluent, Hexane/AcOEt $=9 / 1 ; 75 \%$ yield; ${ }^{1} \mathrm{H}$ NMR $(270 \mathrm{MHz}$, $\left.\mathrm{CDCl}_{3}\right): \delta 3.14(\mathrm{t}, J=6.7 \mathrm{~Hz}, 2 \mathrm{H}), 3.39-3.47(\mathrm{~m}, 1 \mathrm{H}), 7.19-7.35(\mathrm{~m}, 5 \mathrm{H}), 7.51-7.63(\mathrm{~m}, 2 \mathrm{H})$, 7.54-7.63 (m, 3H), $8.04(\mathrm{dd}, J=8.7 \mathrm{~Hz}, J=1.87 \mathrm{~Hz}, 1 \mathrm{H}), 8.46(\mathrm{~s}, 1 \mathrm{H}) ;{ }^{13} \mathrm{C}$ NMR $(67.5 \mathrm{MHz}$, $\left.\mathrm{CDCl}_{3}\right): \delta 30.3,40.2(\mathrm{t}, J=19.5 \mathrm{~Hz}), 40.5,123.9,126.2,126.8,127.8,128.4,128.5,129.5$, 129.7, 132.6, 134.2, 135.6, 141.3, 199.2; HRMS (EI): calcd for $\mathrm{C}_{19} \mathrm{H}_{15} \mathrm{DO}$ : 261.1264; found 261.1277 . 
4. NMR spectra

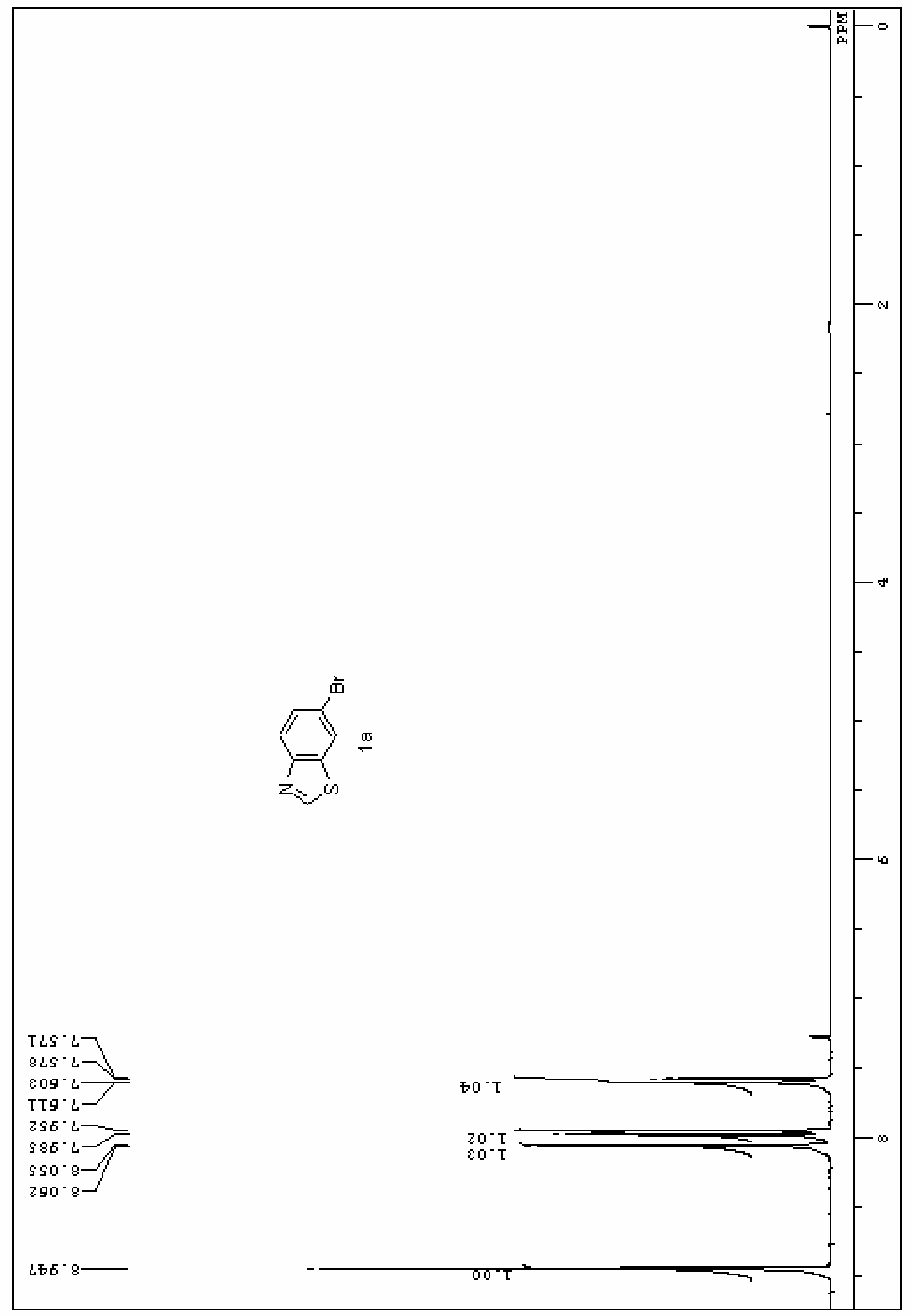




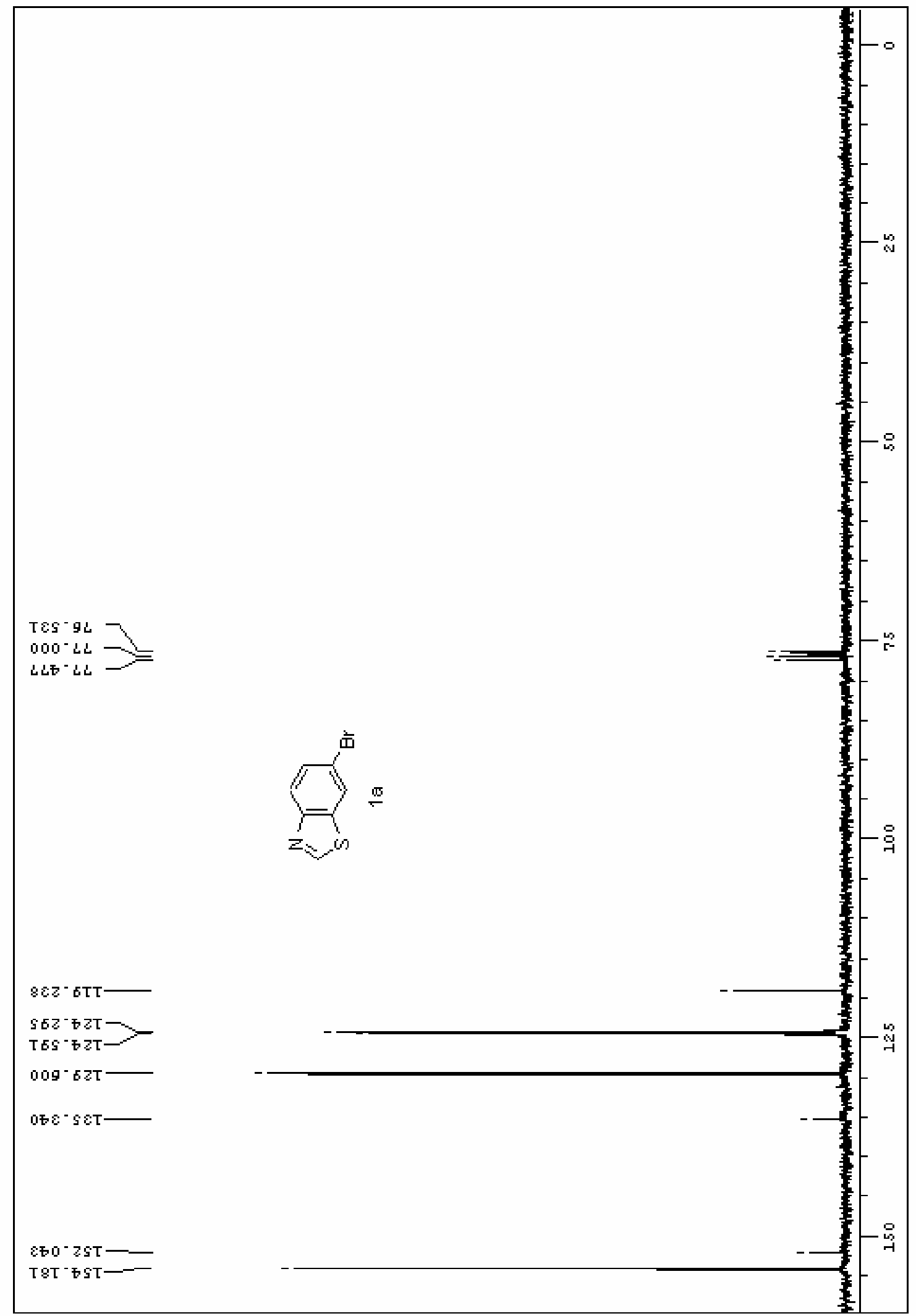




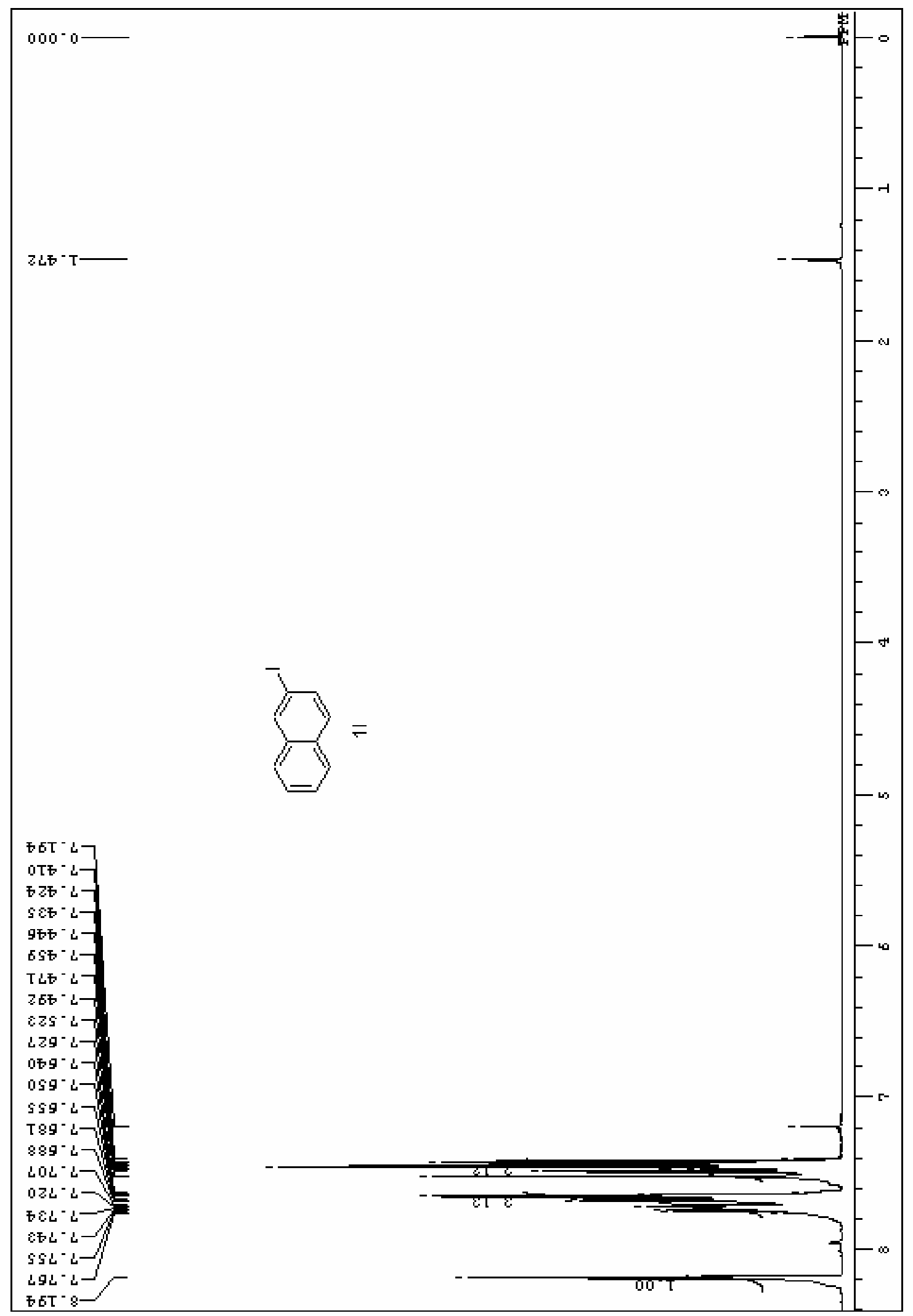




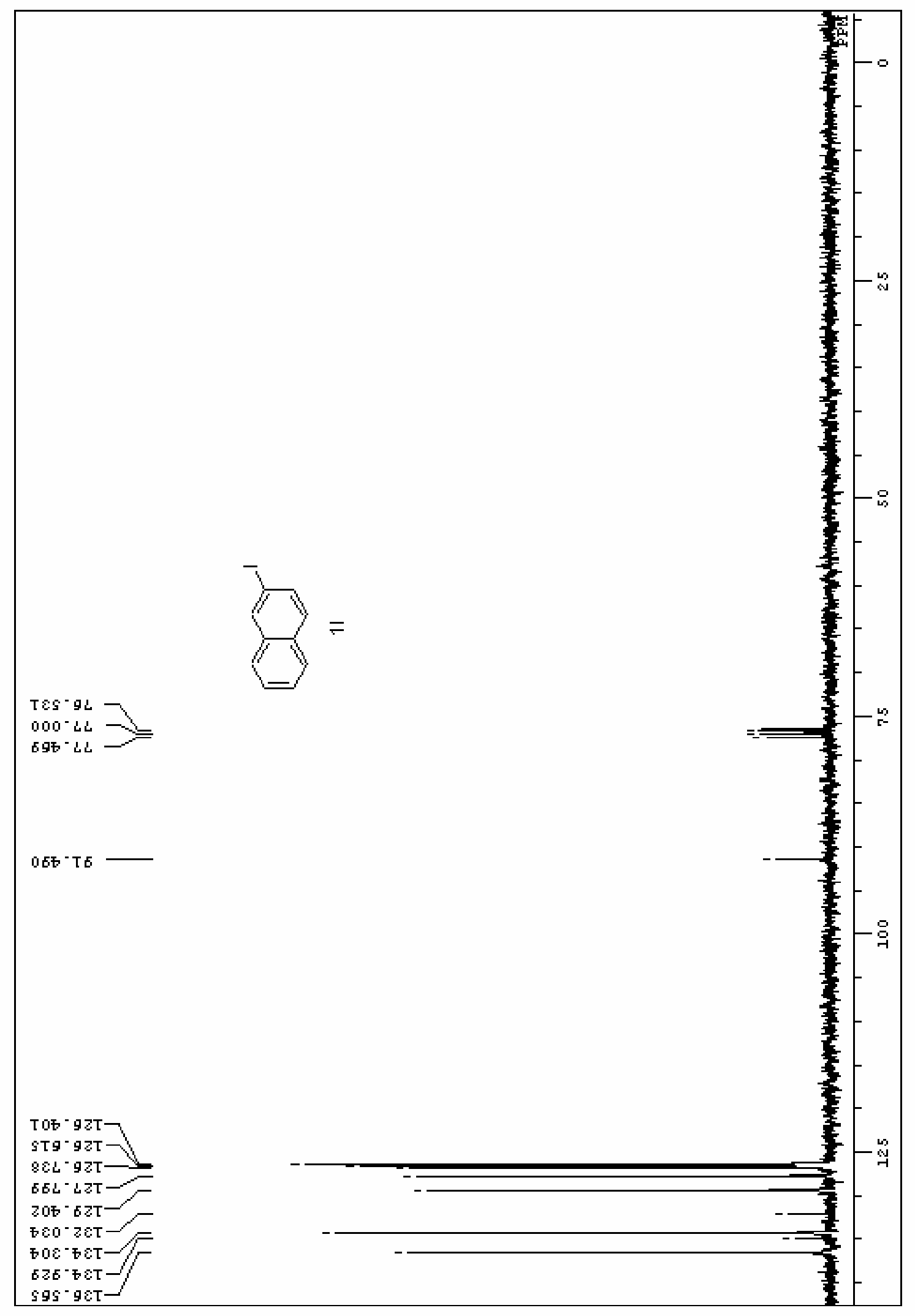




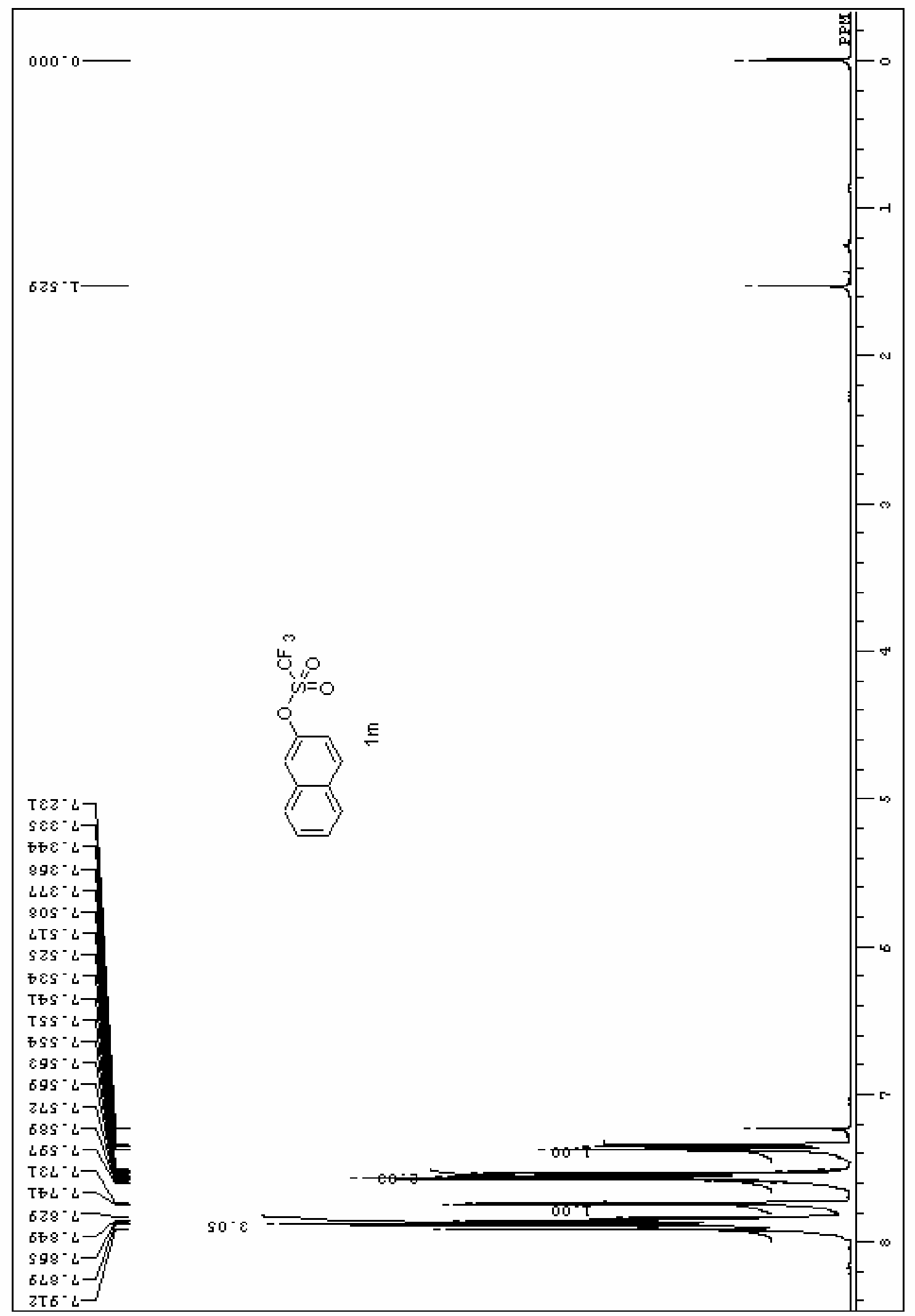




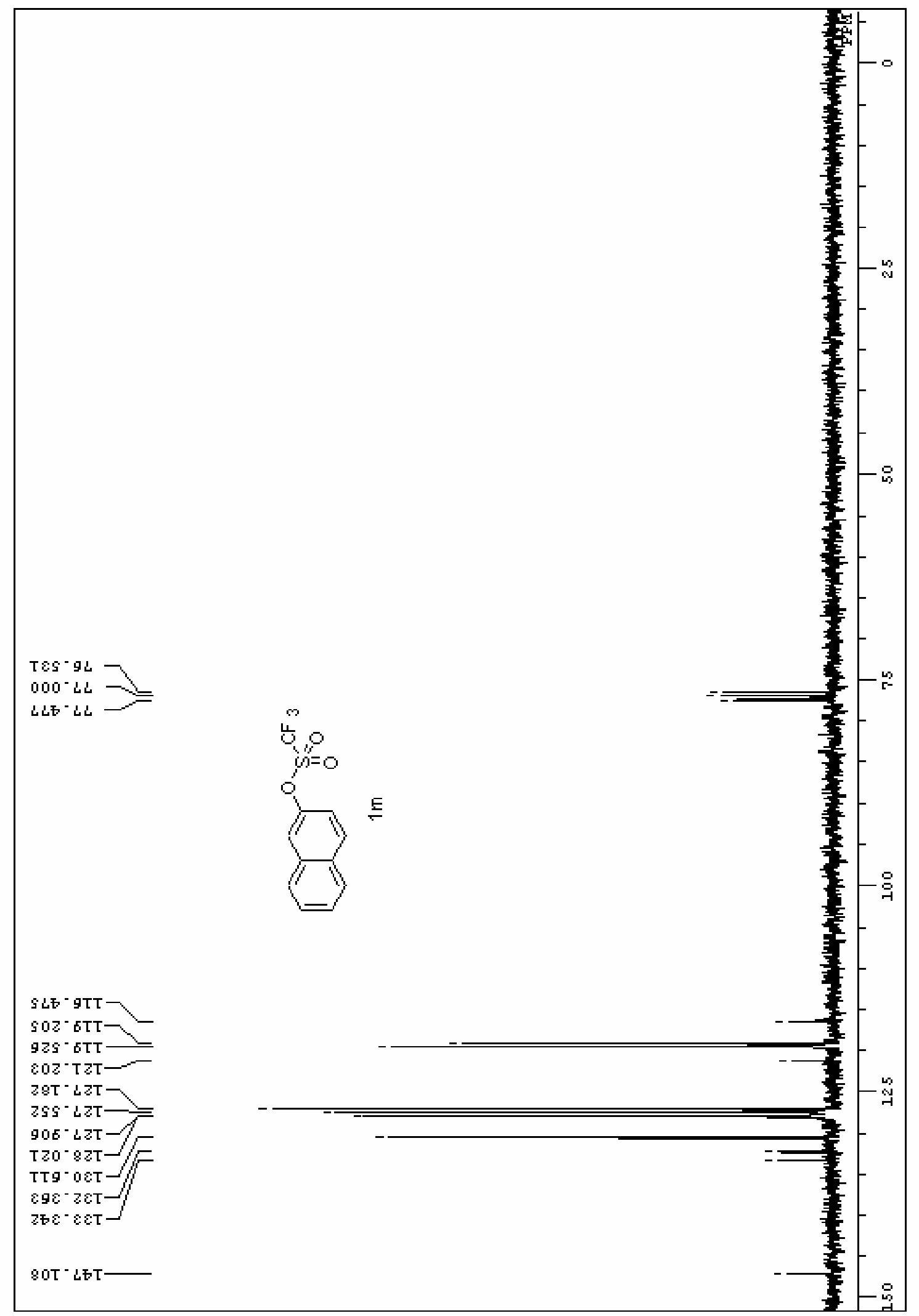




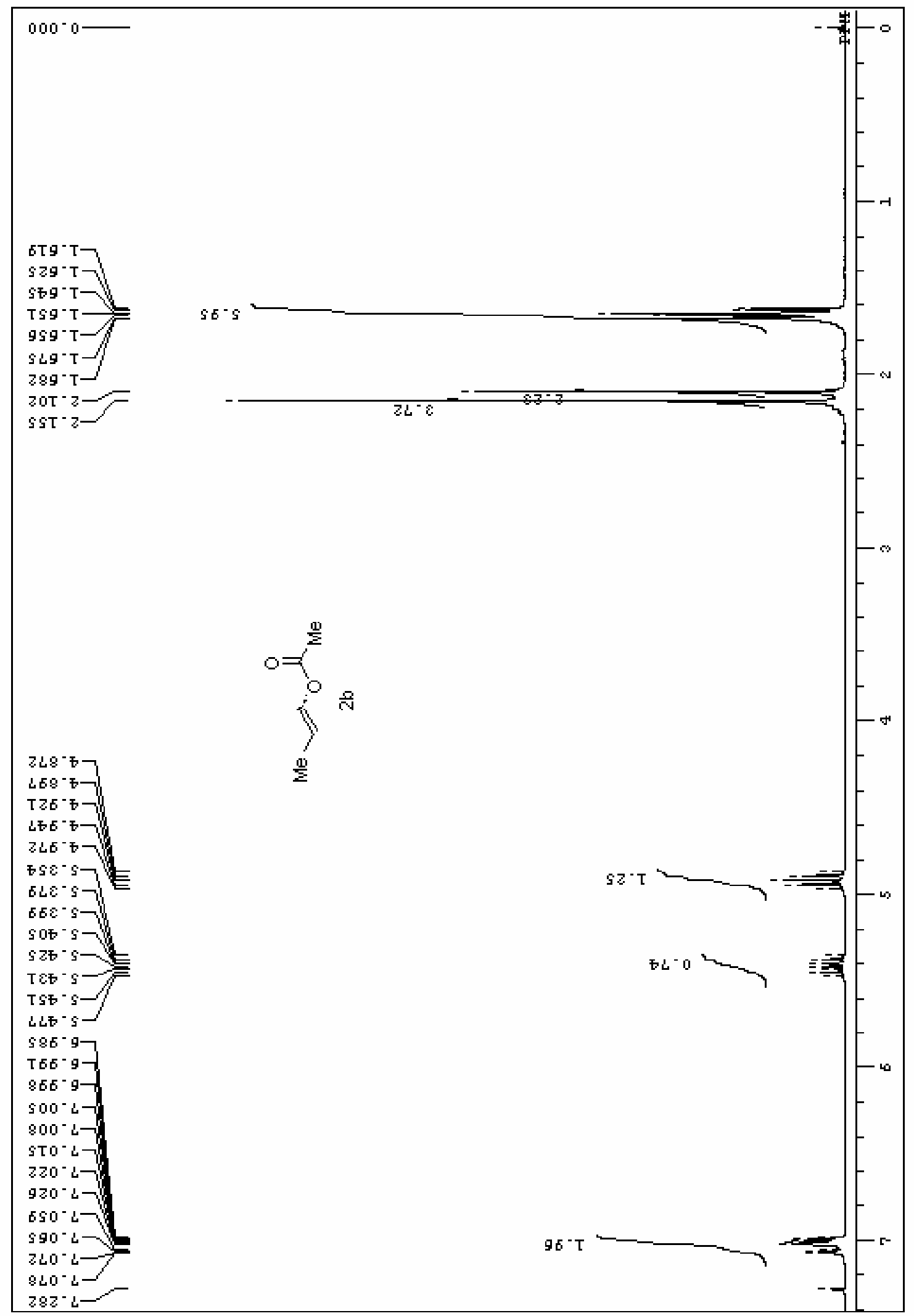




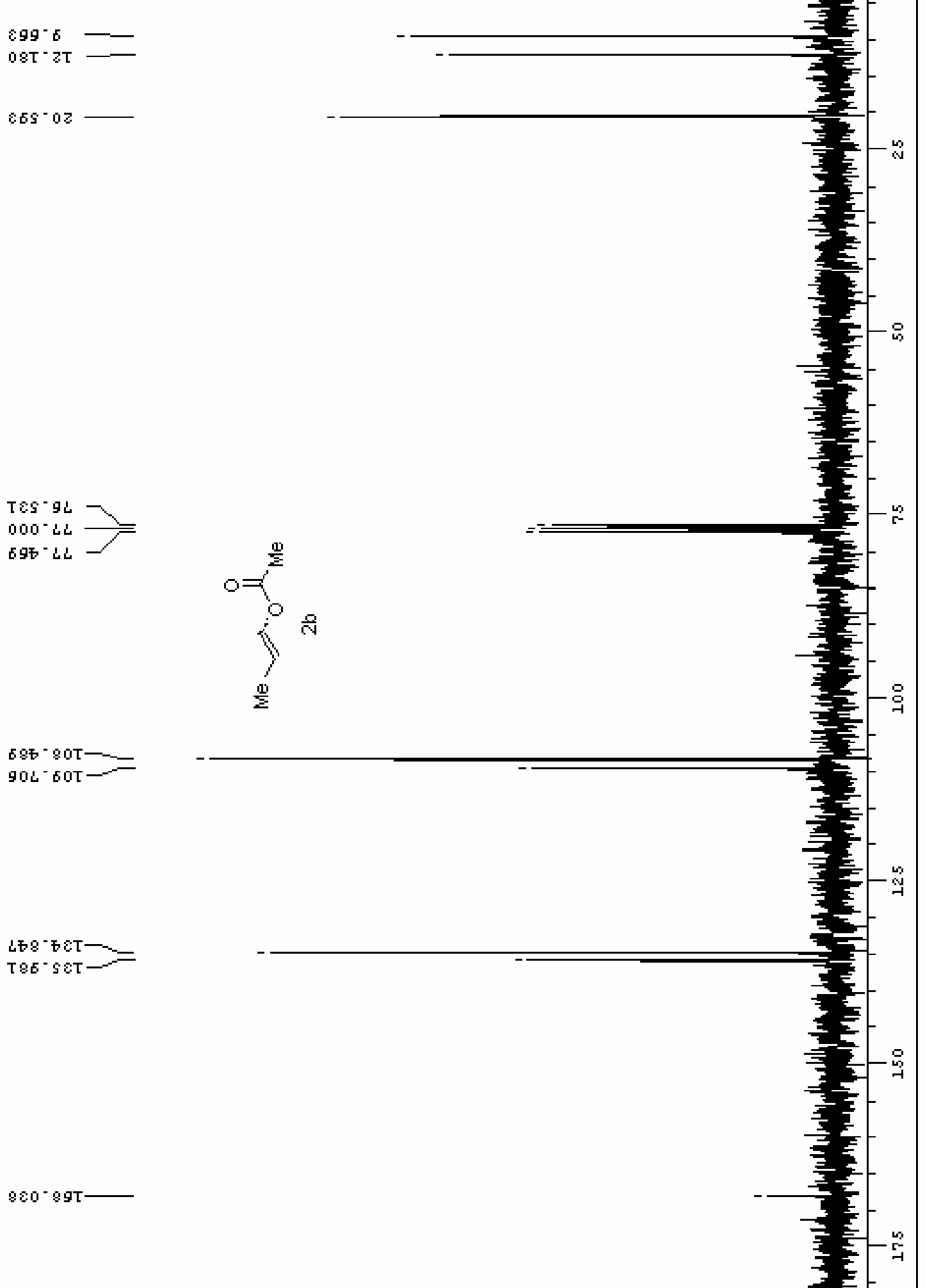




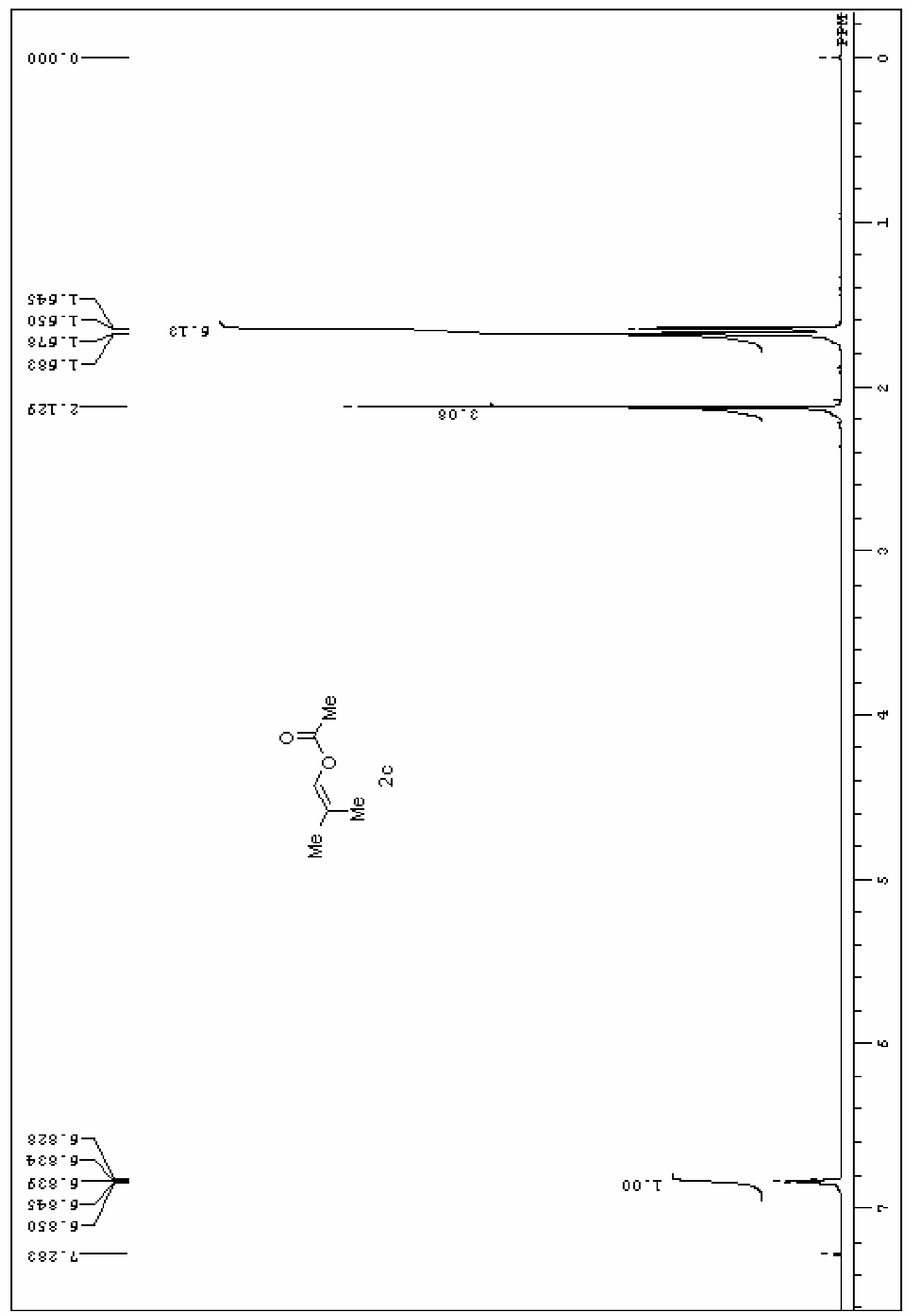




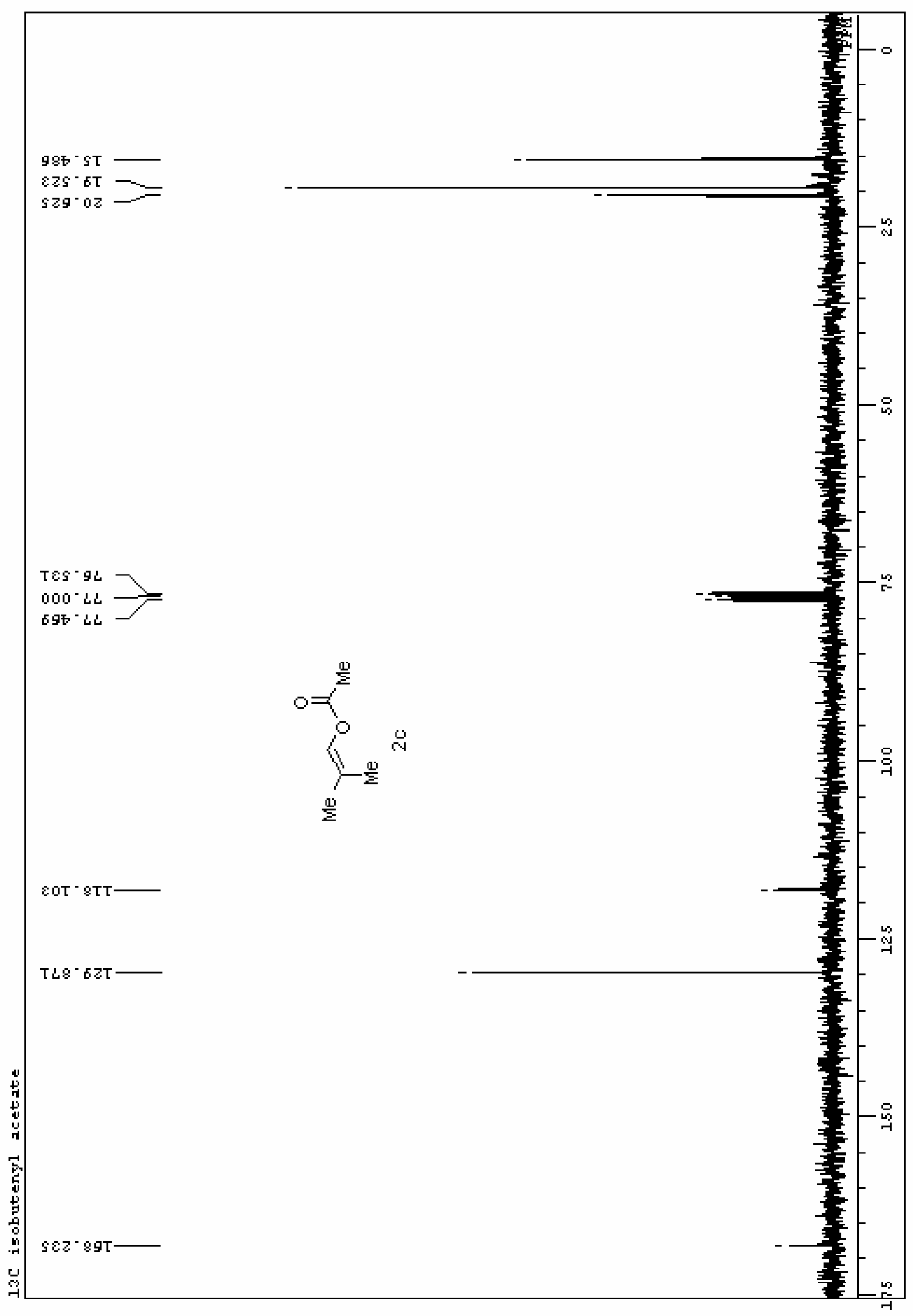

S21 


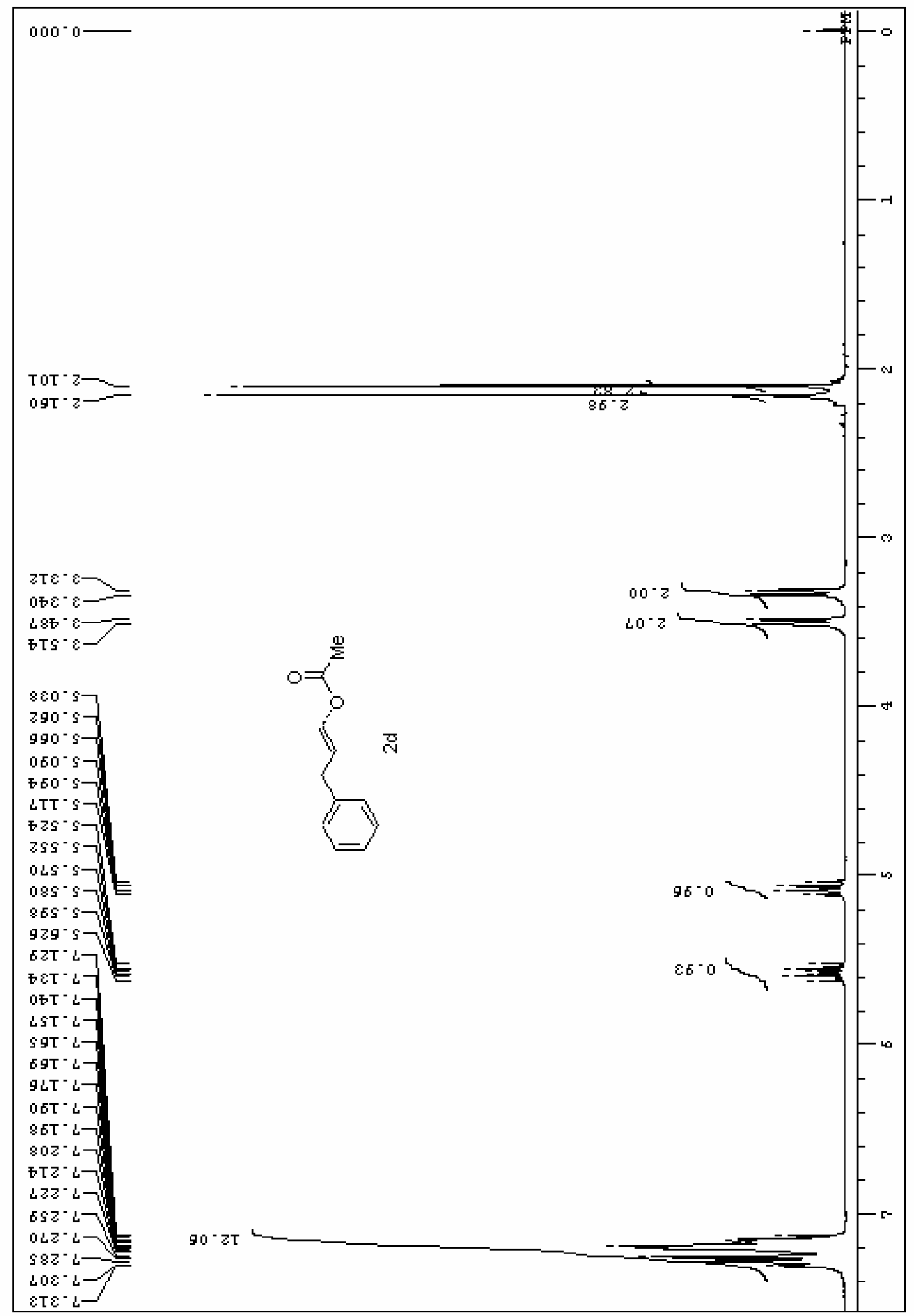




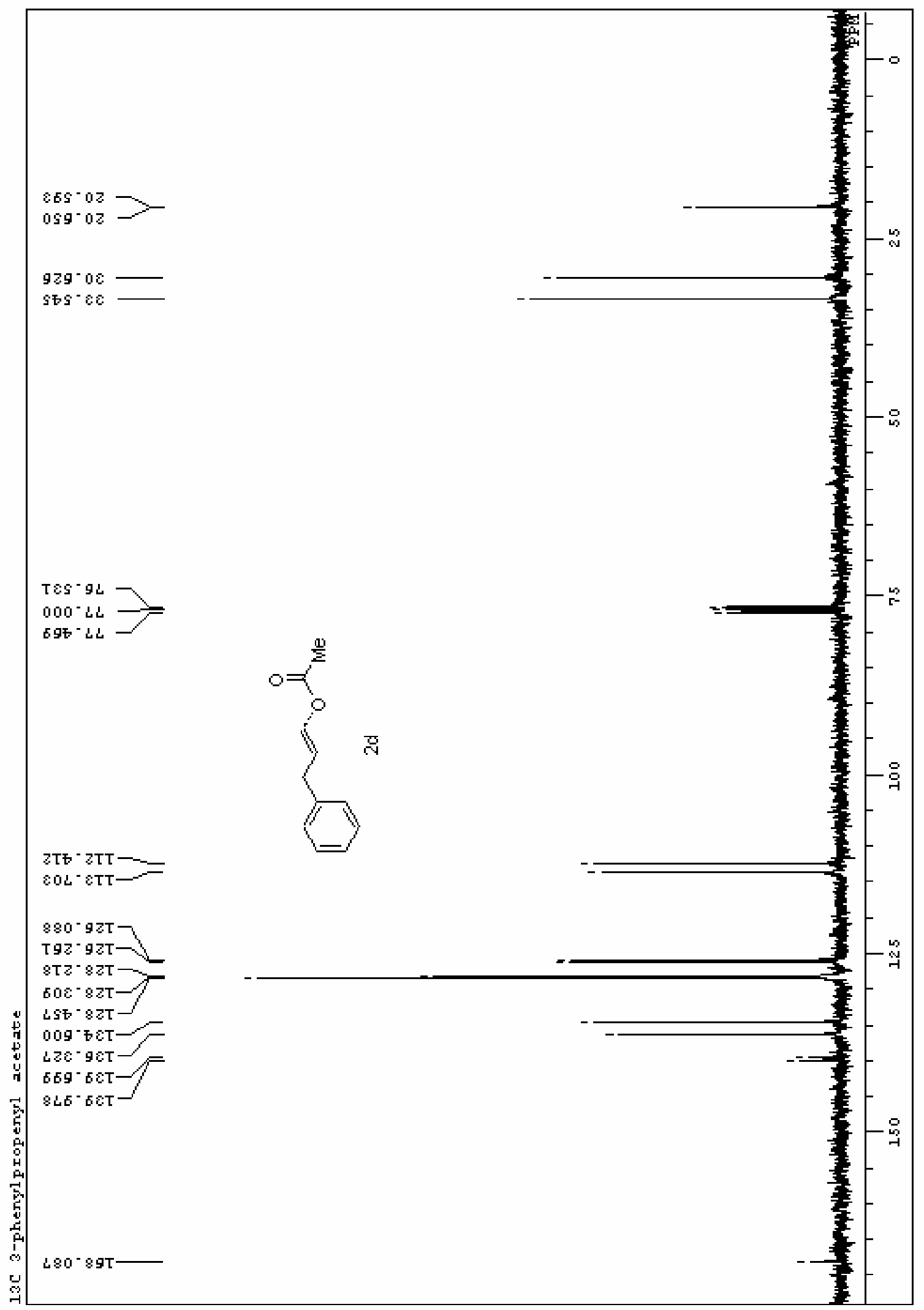




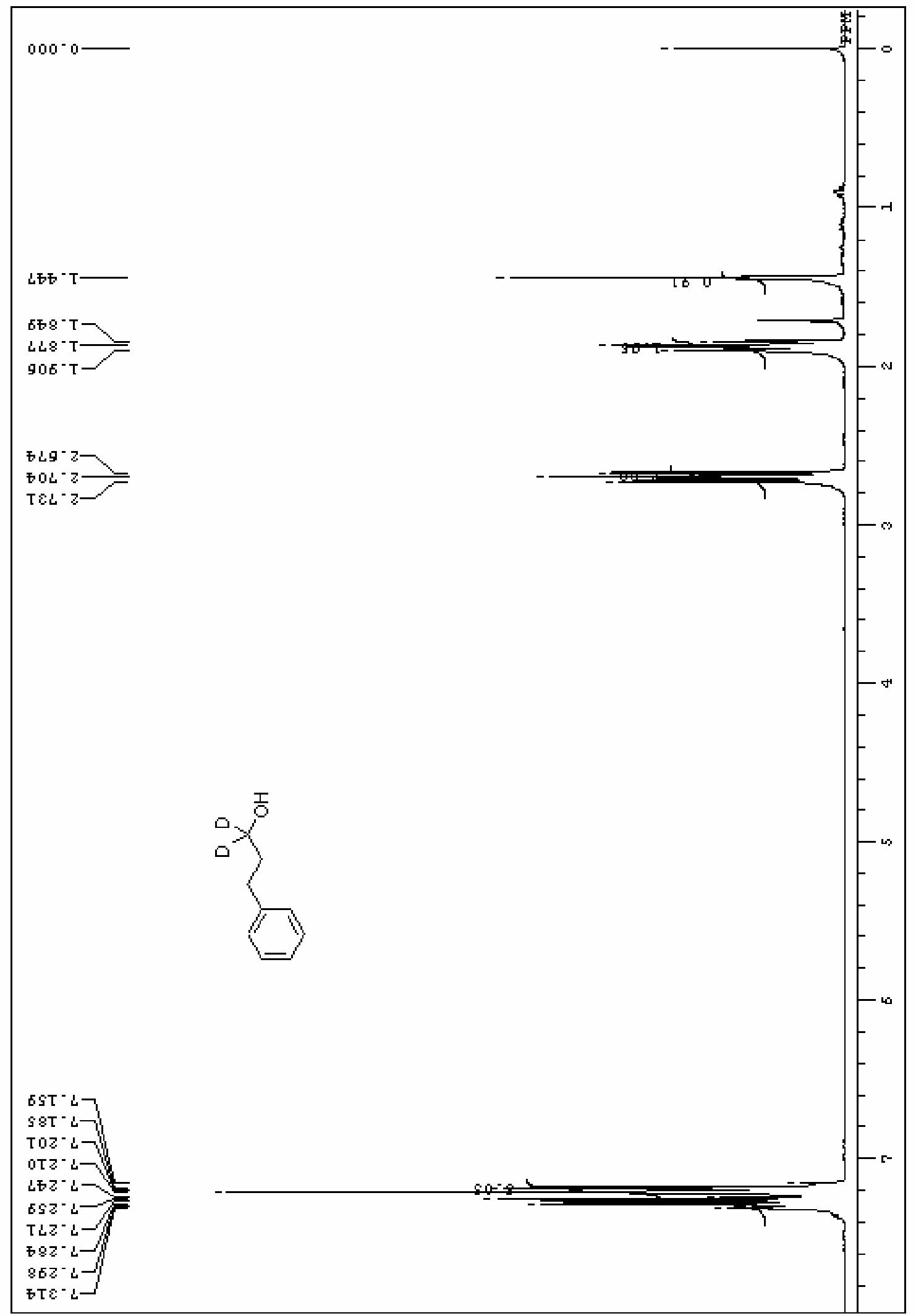




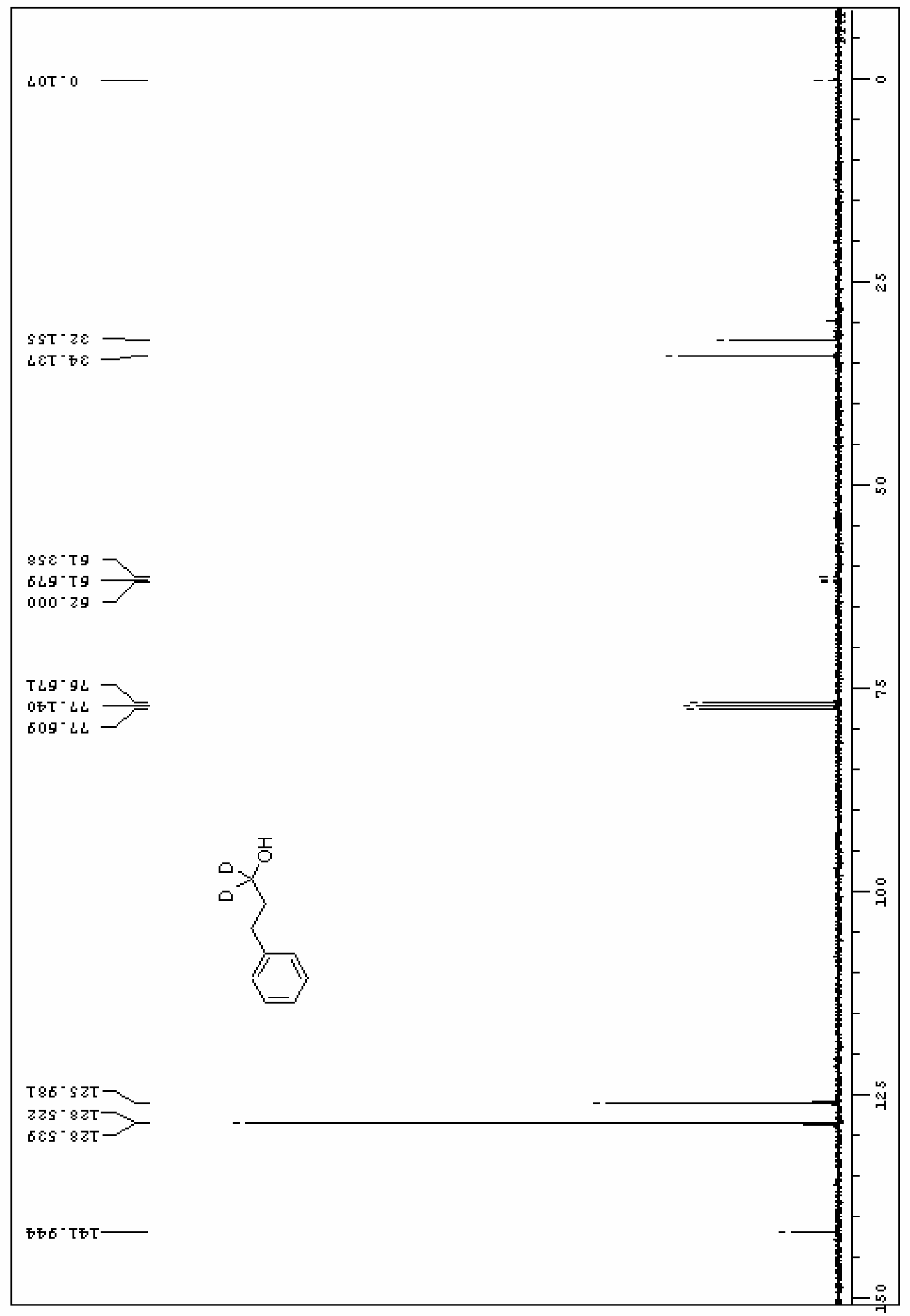




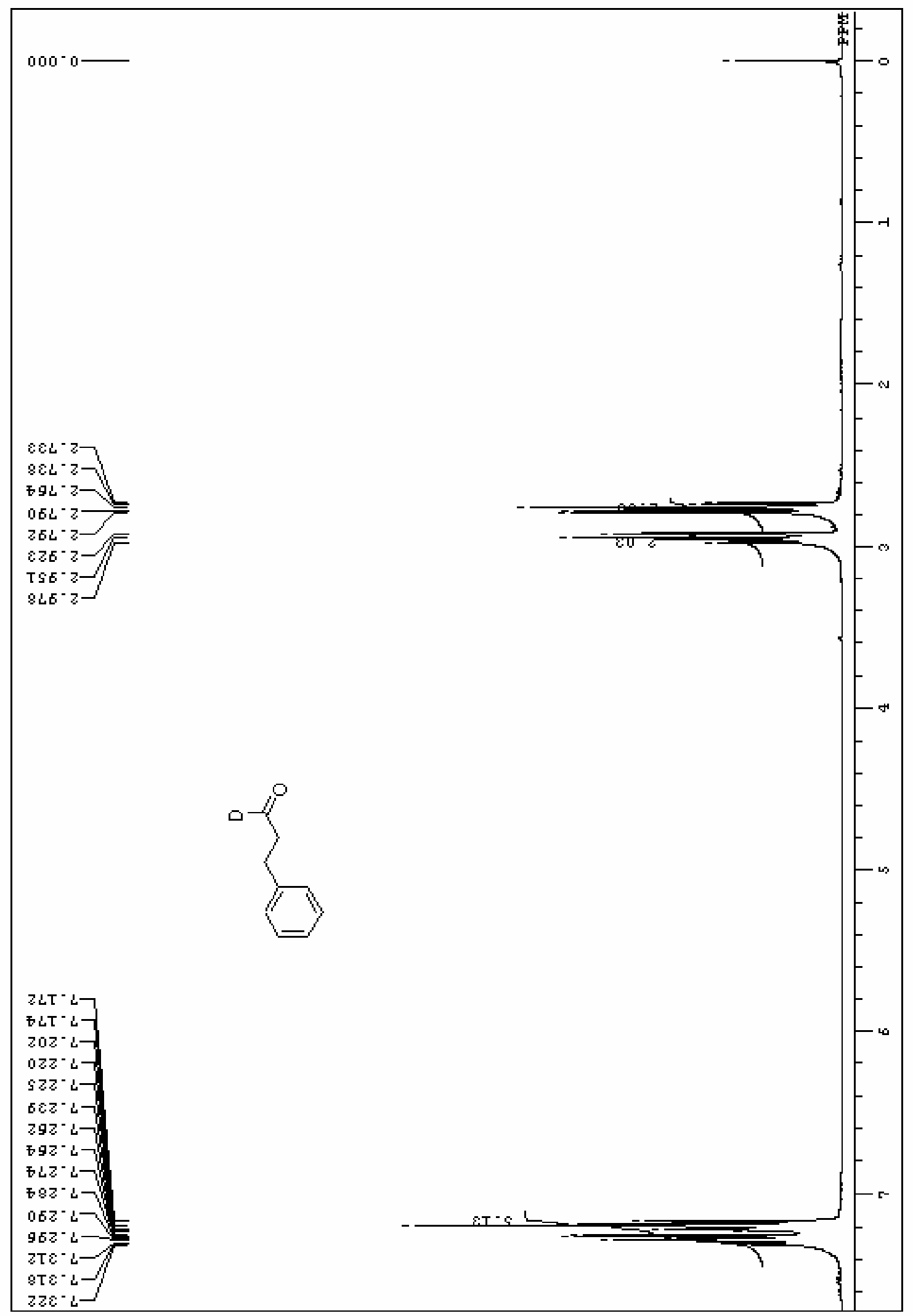




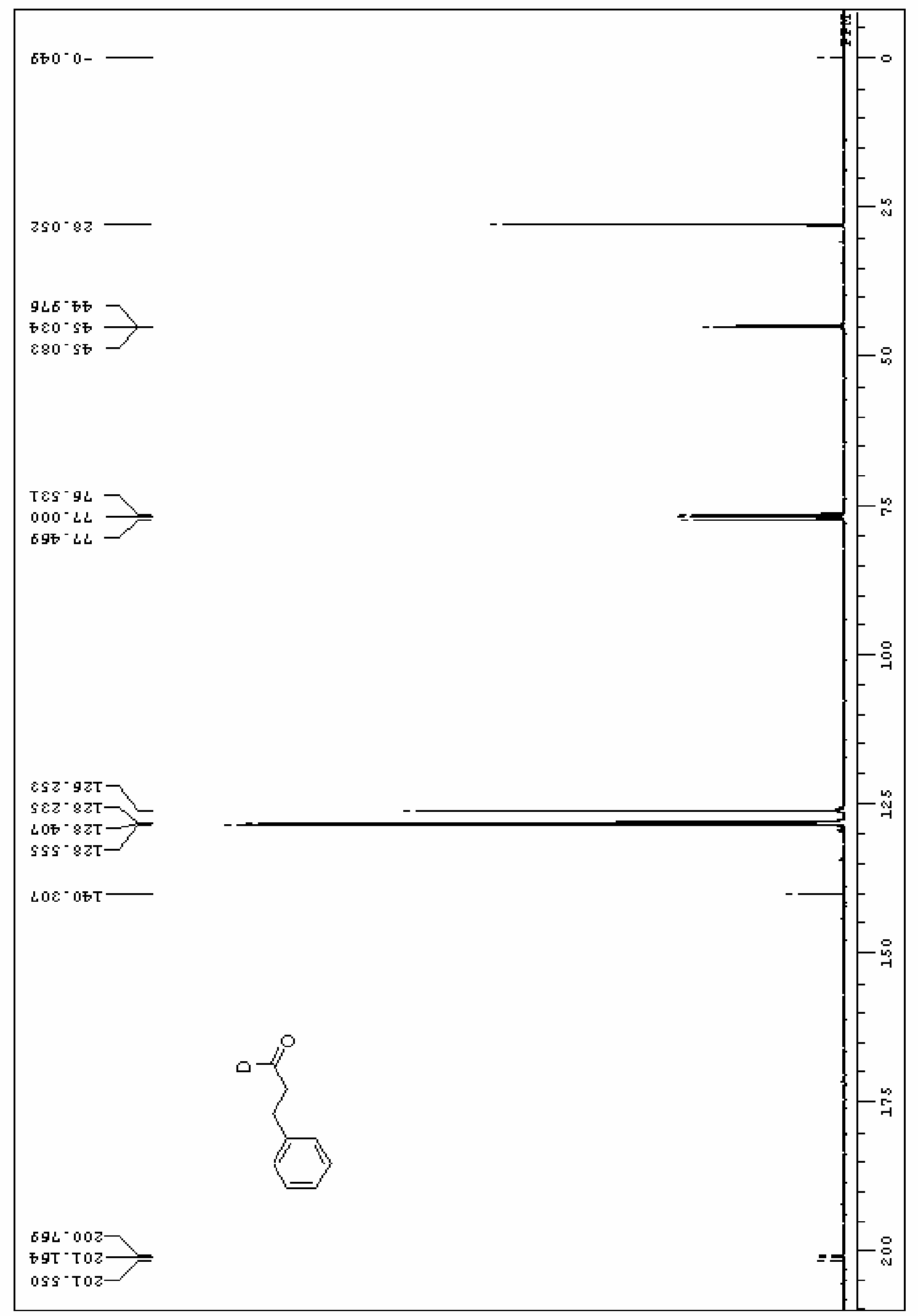




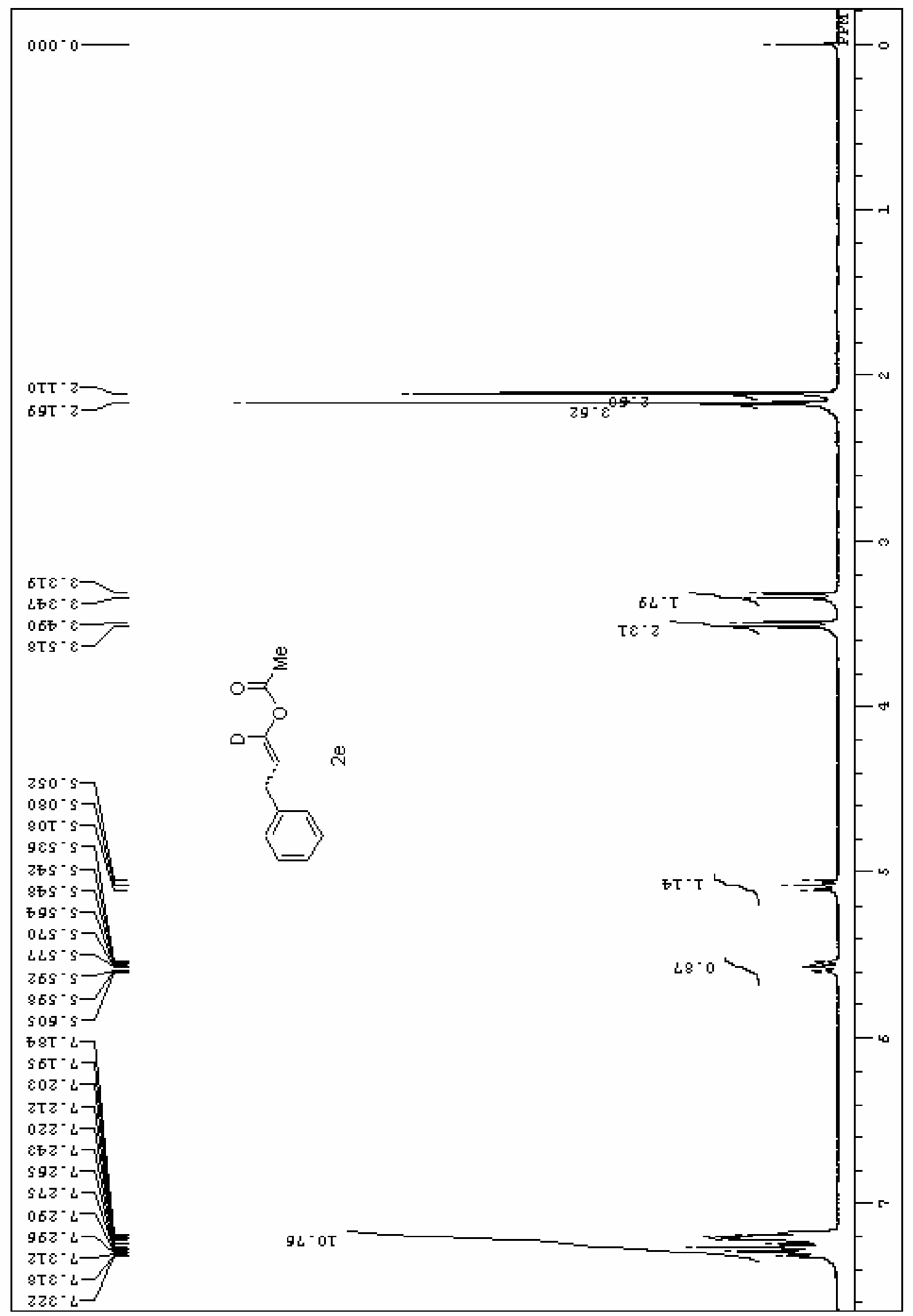


七8- 02

cog-0

LTg- 08

$\operatorname{Lsg} 8$

Teg-gL

000-L它

ししもーしし

$602^{-2 \mathrm{TT}}-$

$\mathrm{sg}^{-6 \mathrm{tT}--}$

$982-92 \mathrm{~T}-$

6क $z^{-} \mathrm{et}$

$528-92 \mathrm{~T}-$

8L五 $92 \mathrm{~T}$

zक
sut

七т - ст-

Lᄂg- $5 \mathrm{ct}-\mathrm{f}$

s0T $98 \mathrm{~T}-\mathrm{h}$

玉 $25-90 \mathrm{~T}$

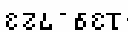

โโ

†95 - ᄂ9T

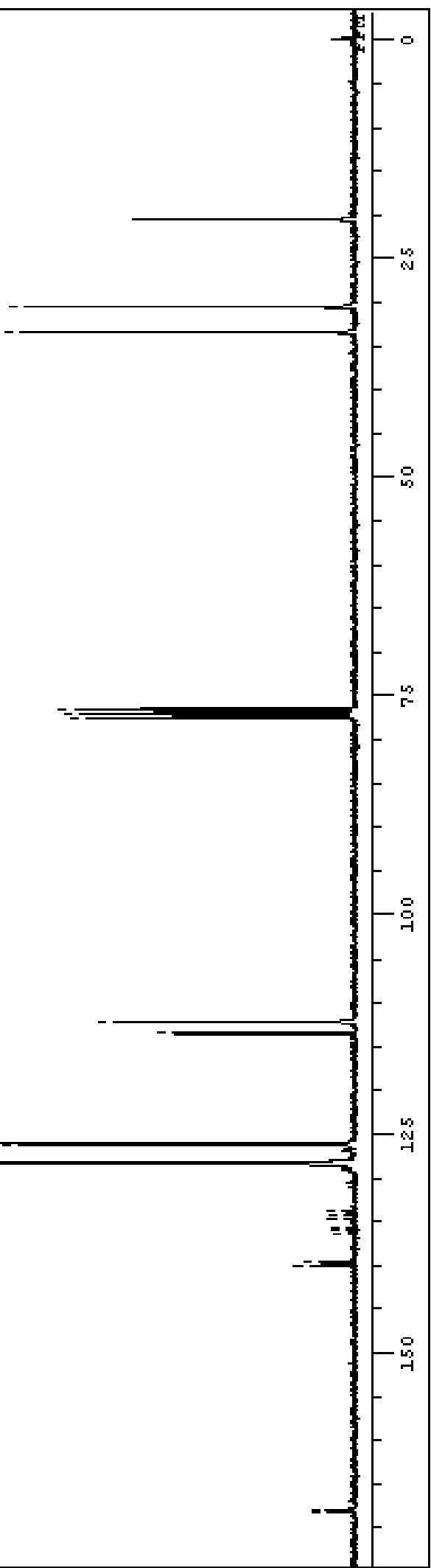




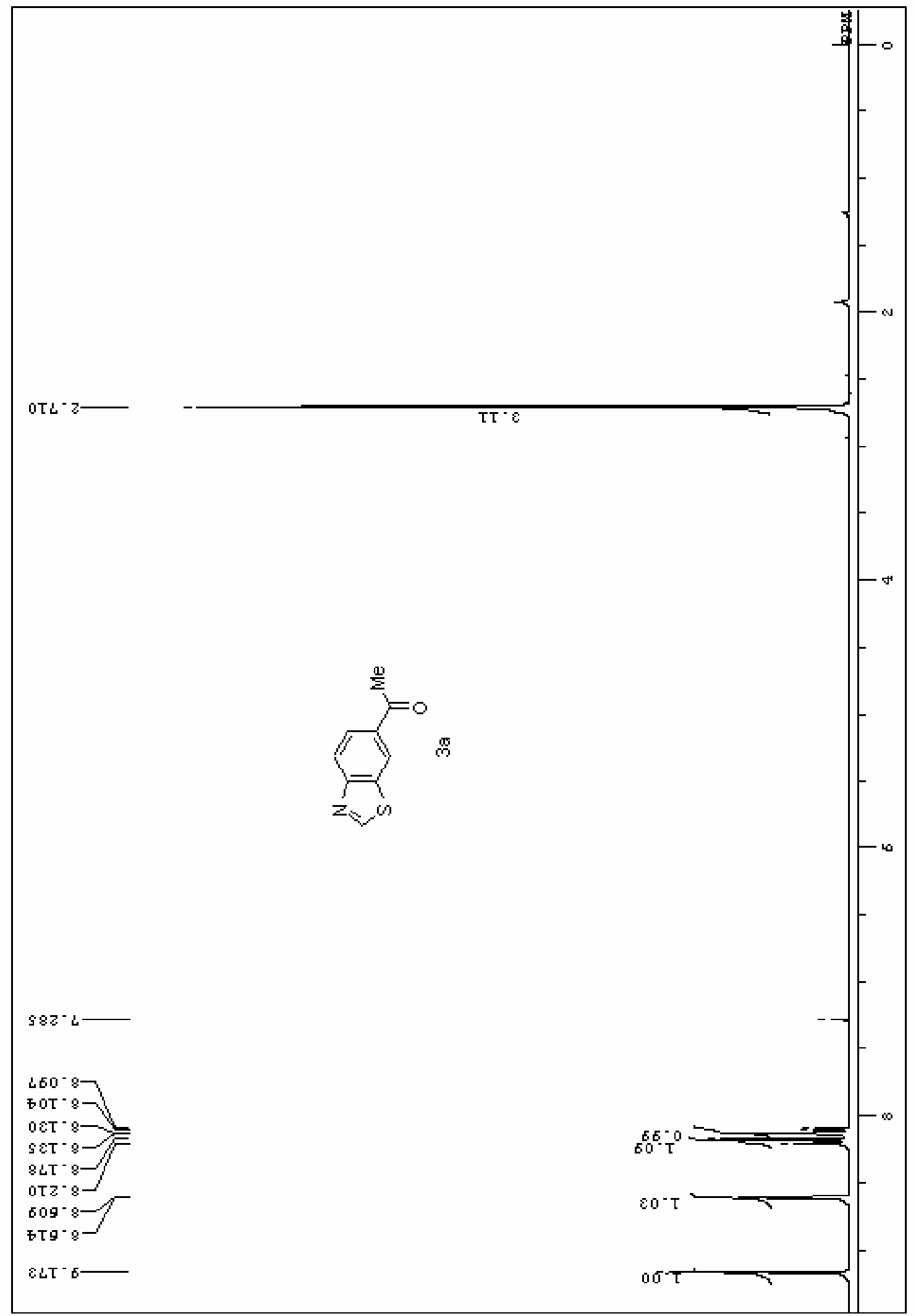




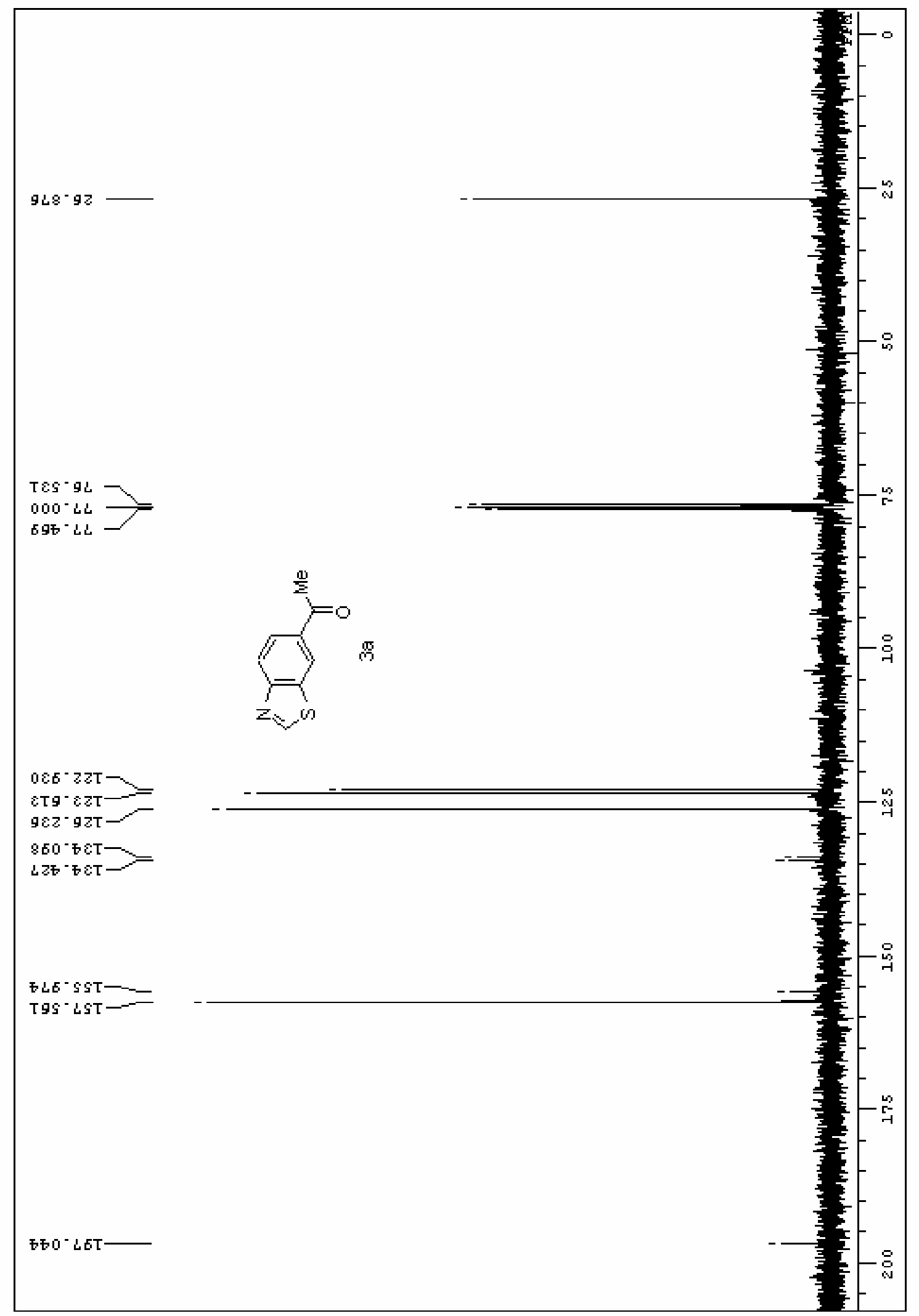




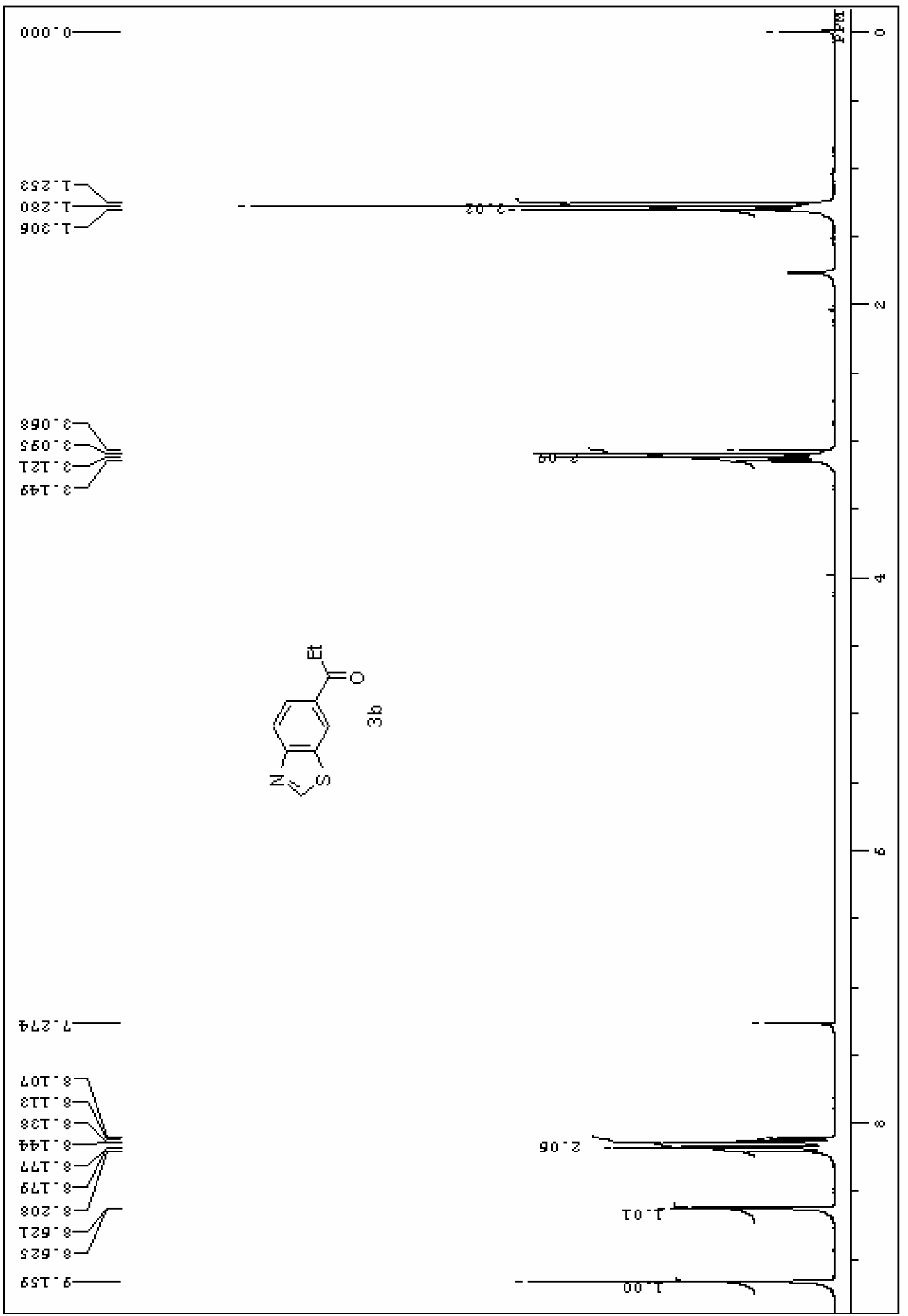




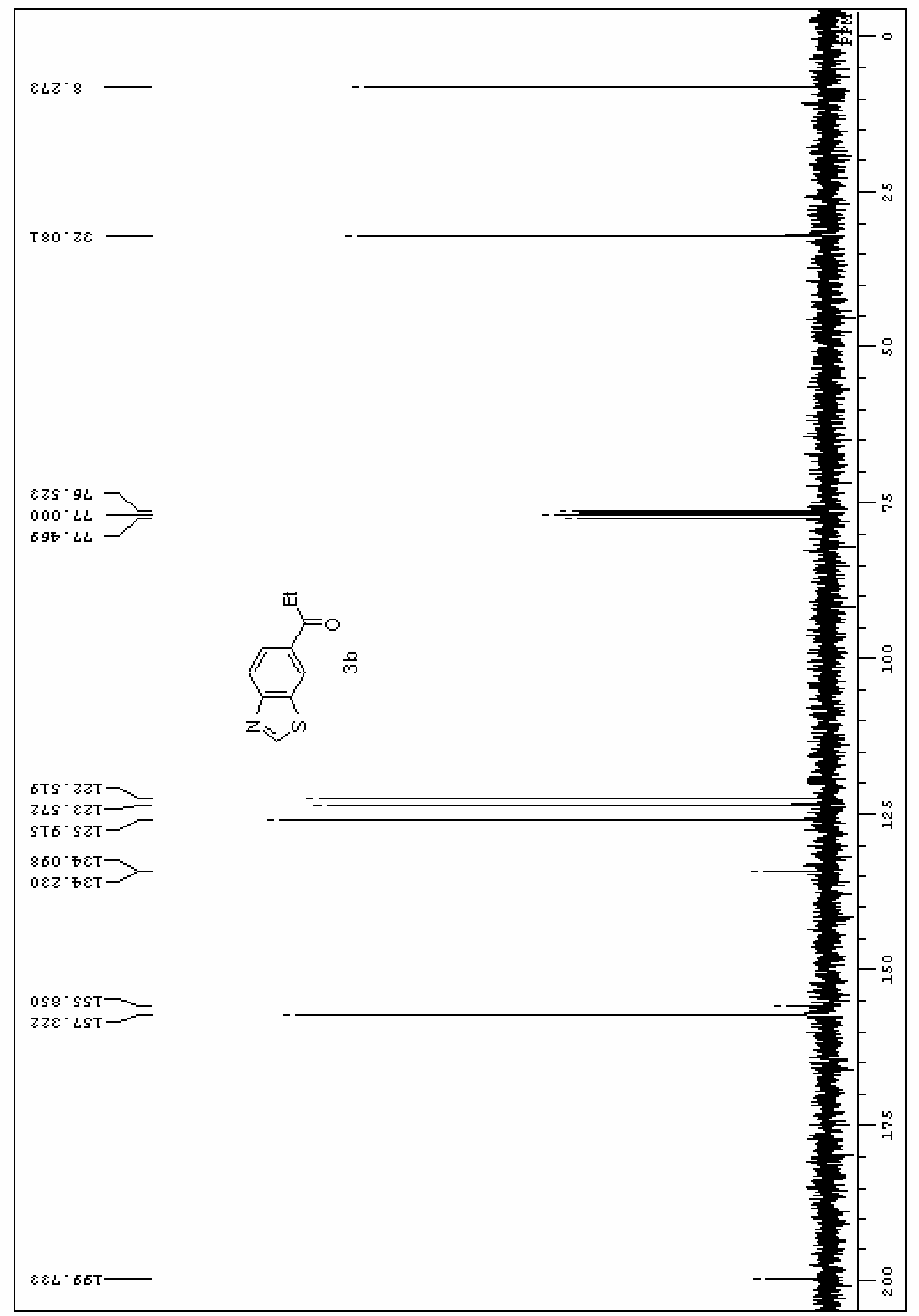




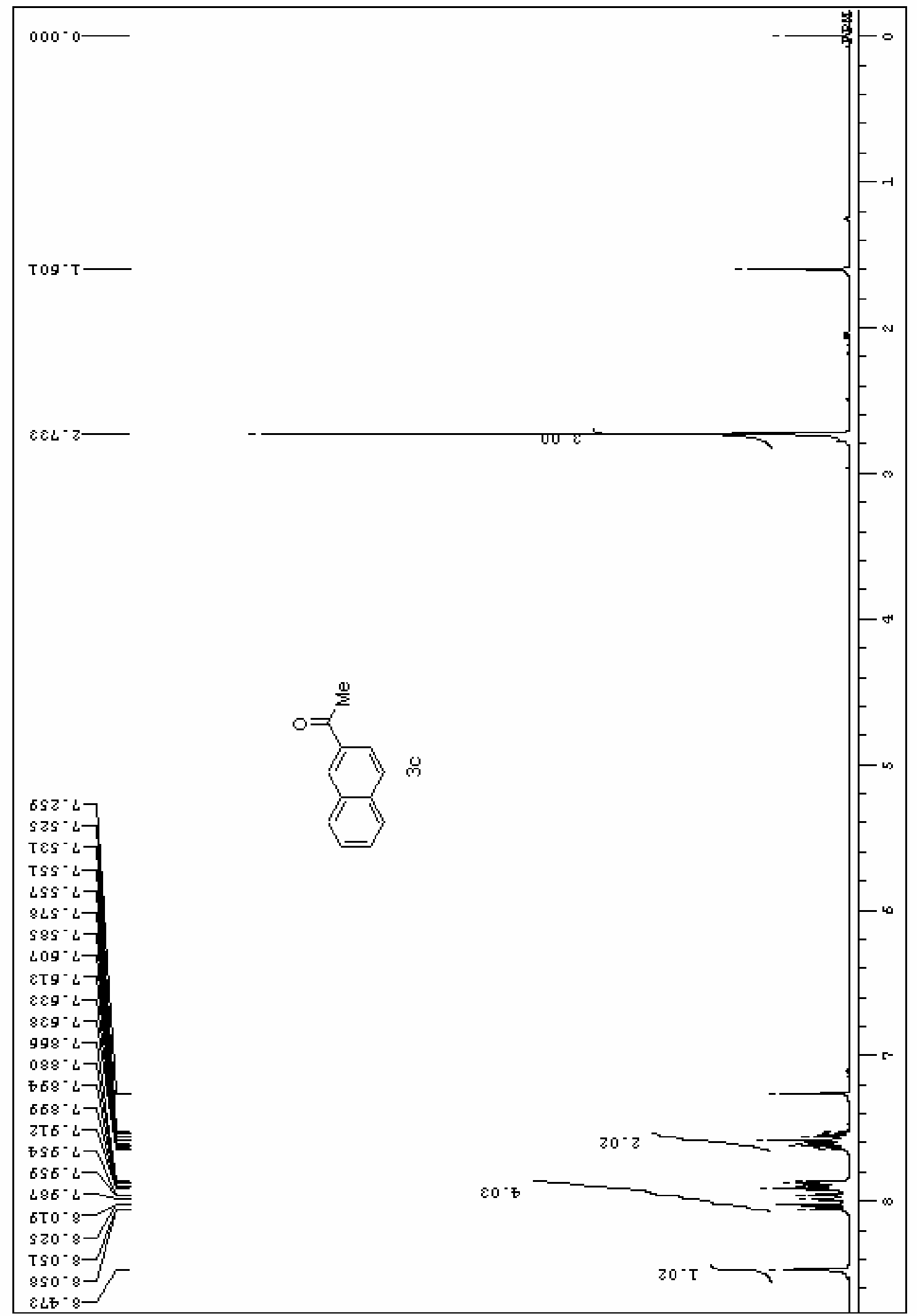




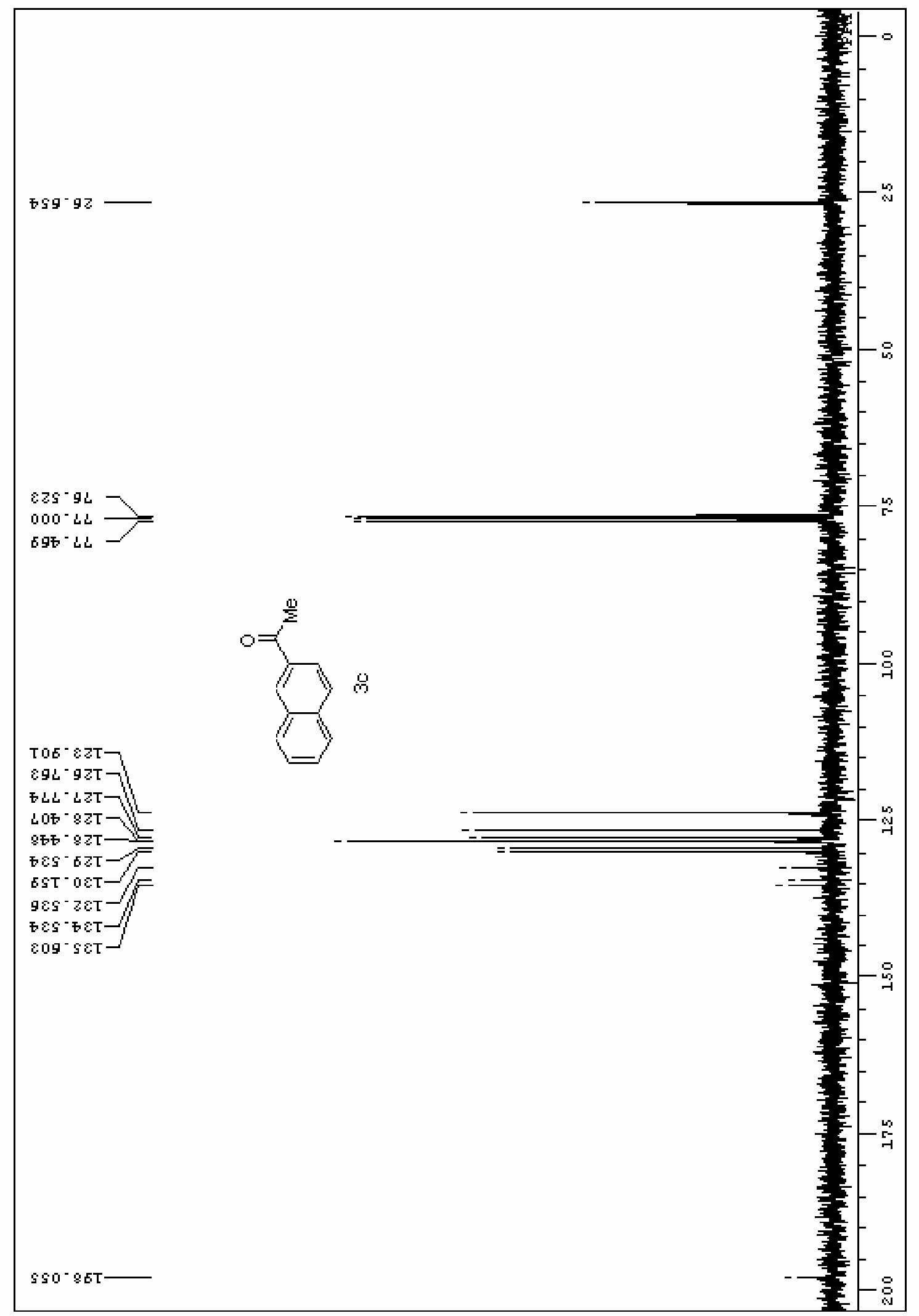




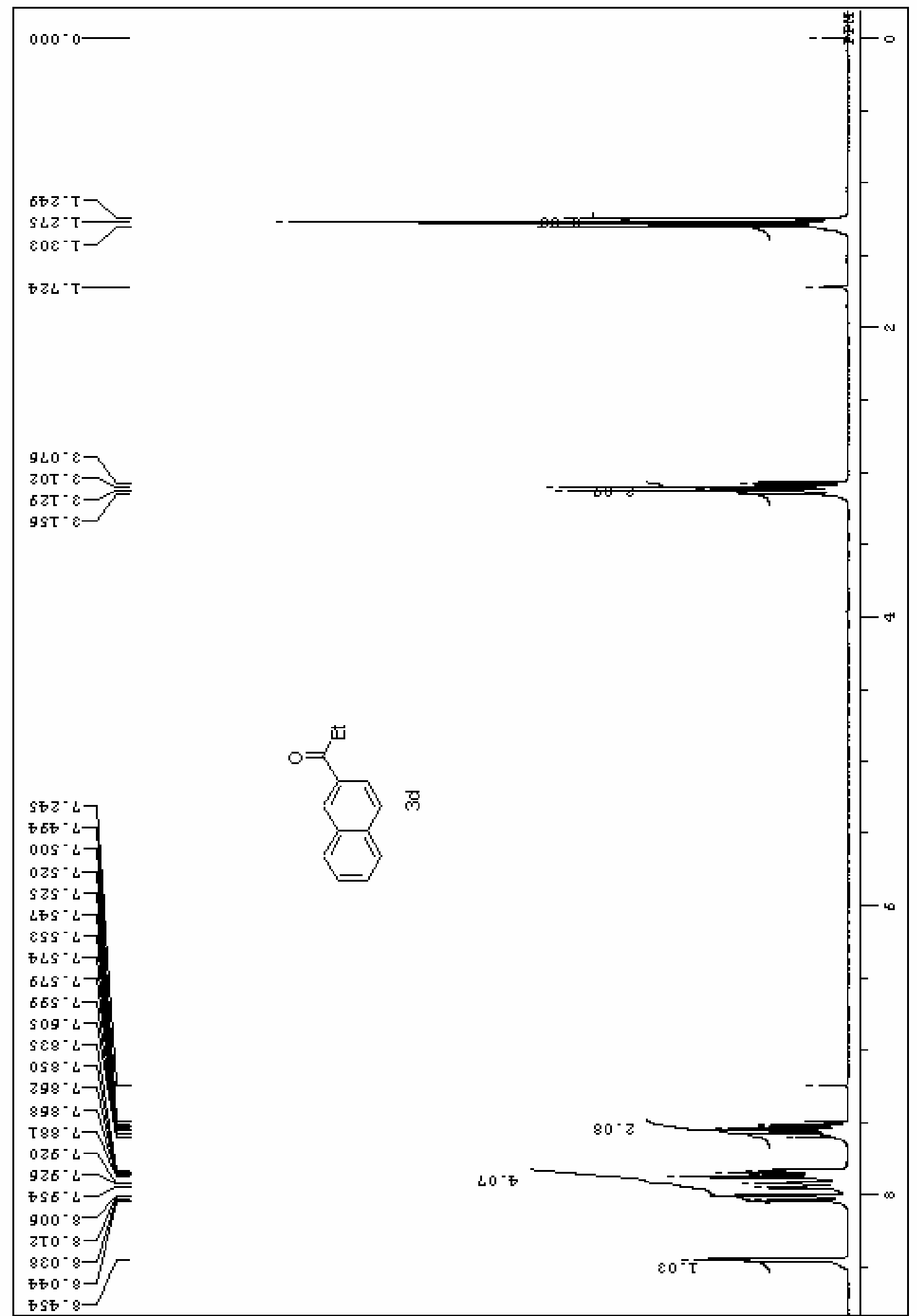




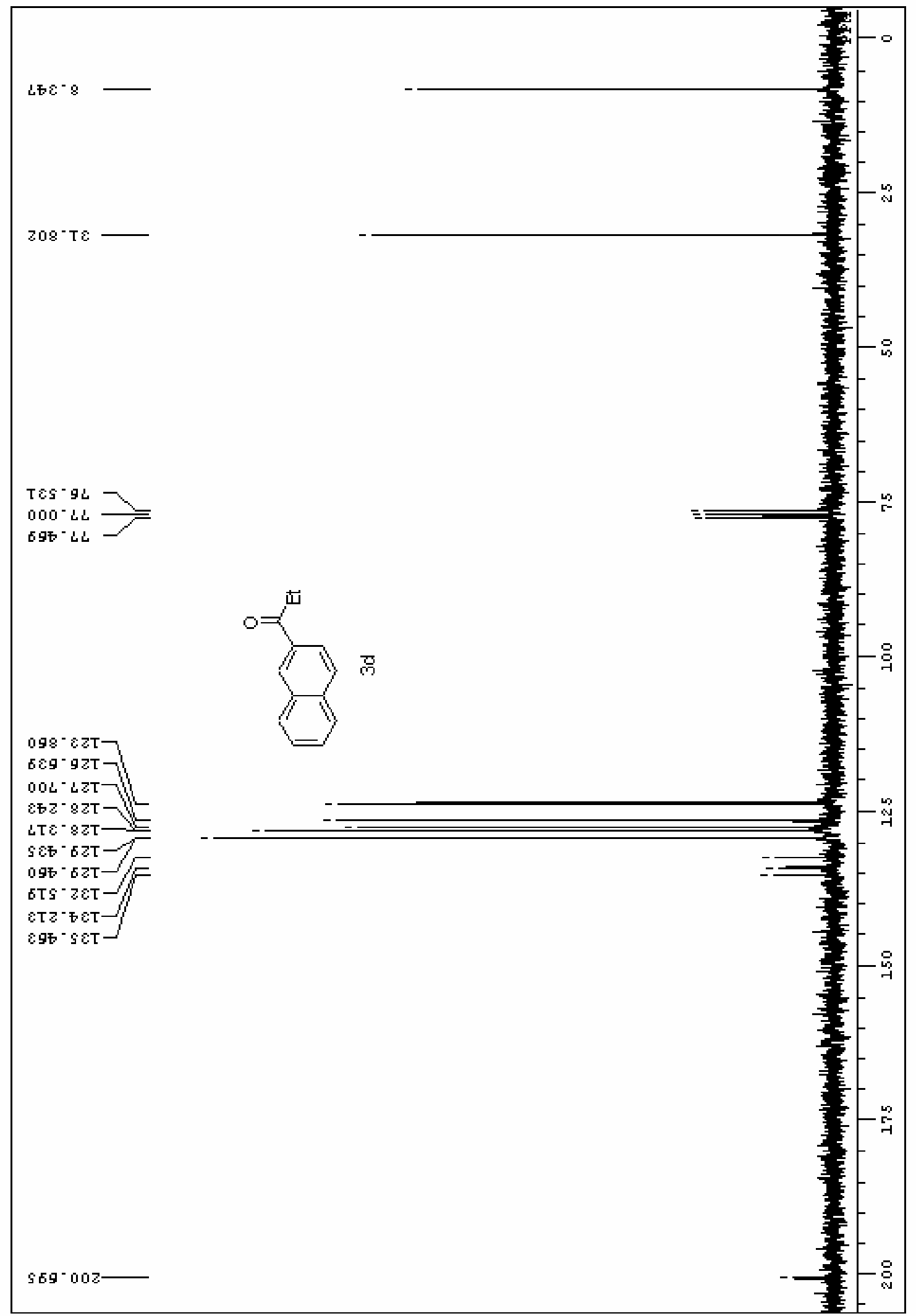




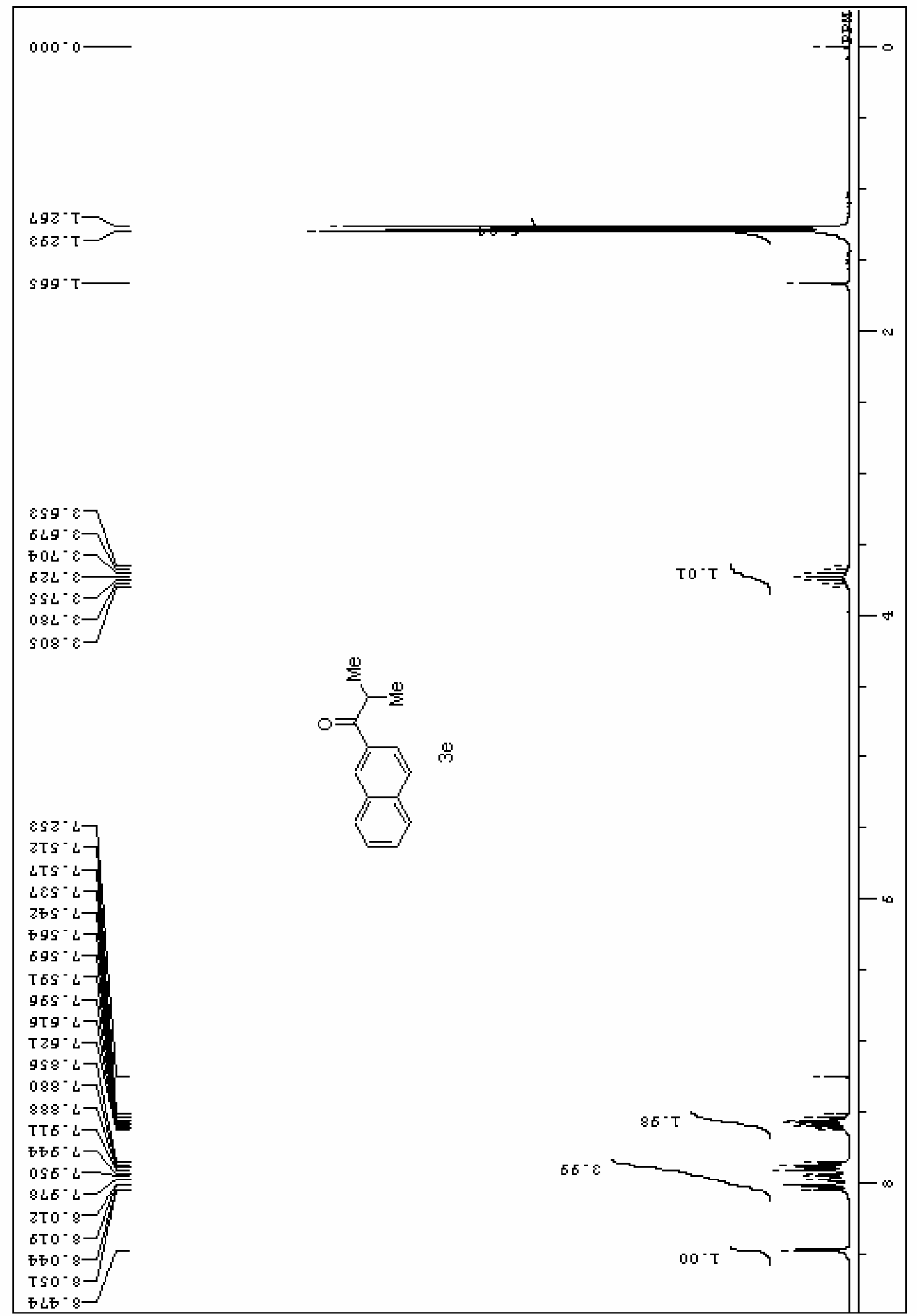




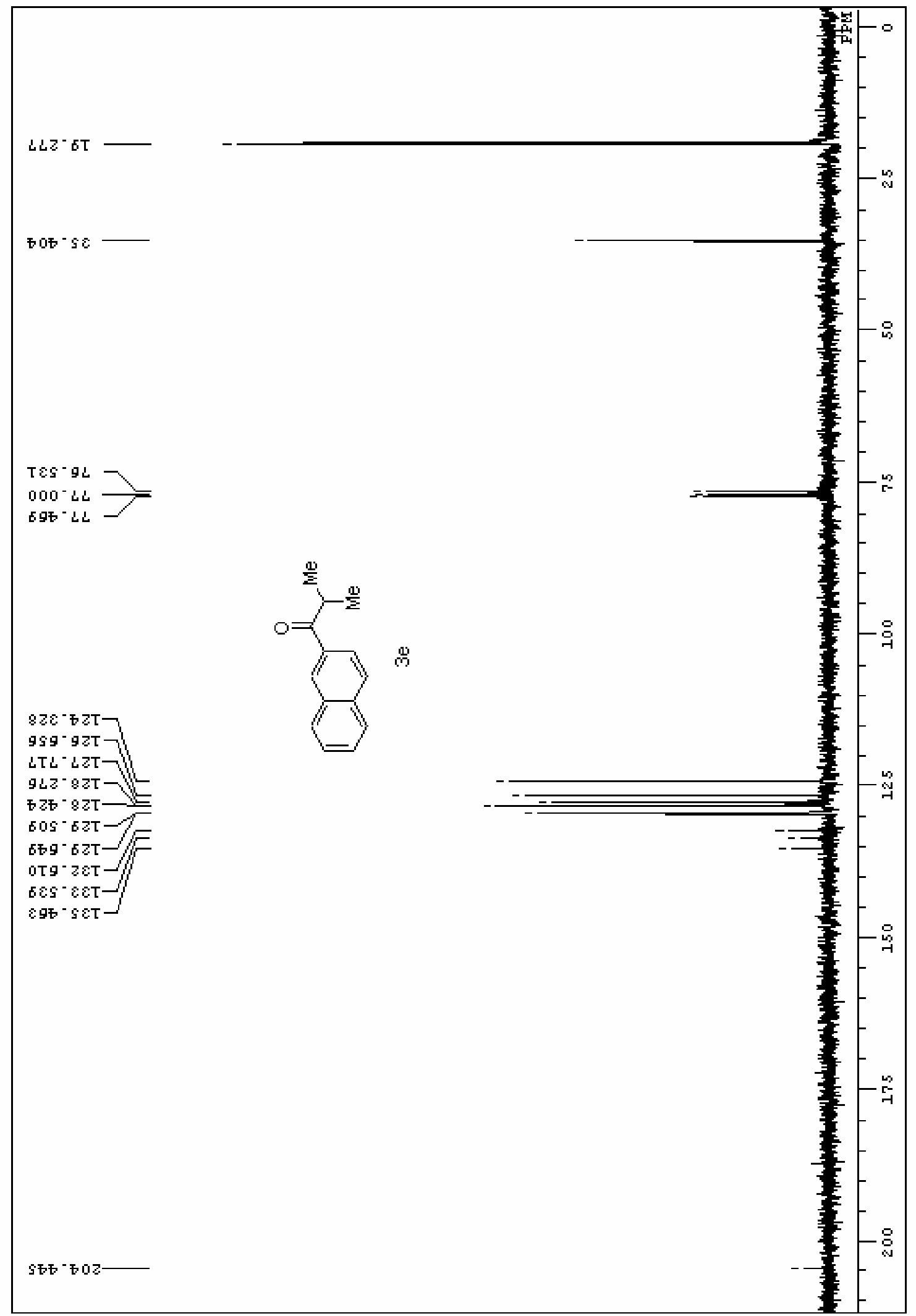




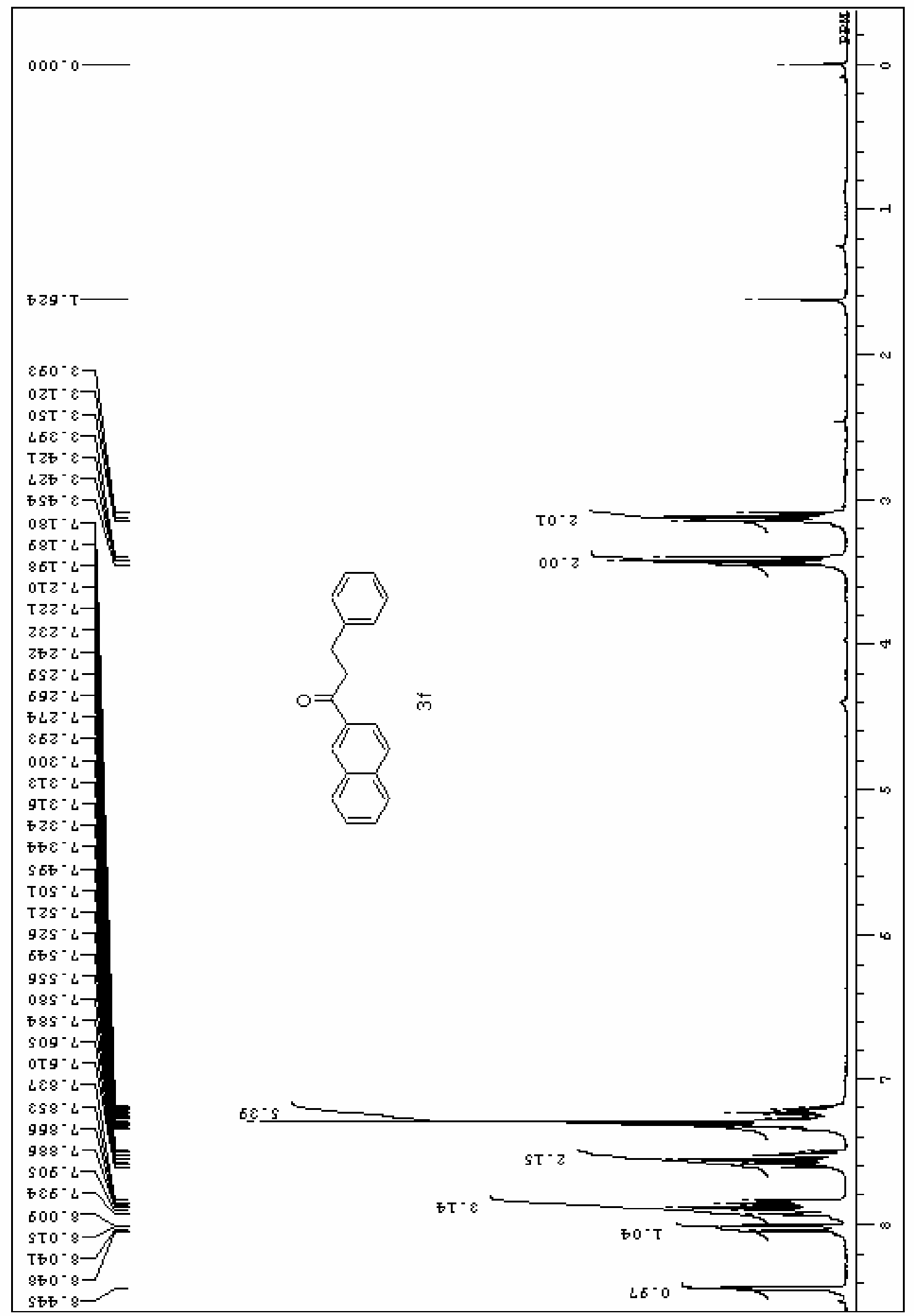




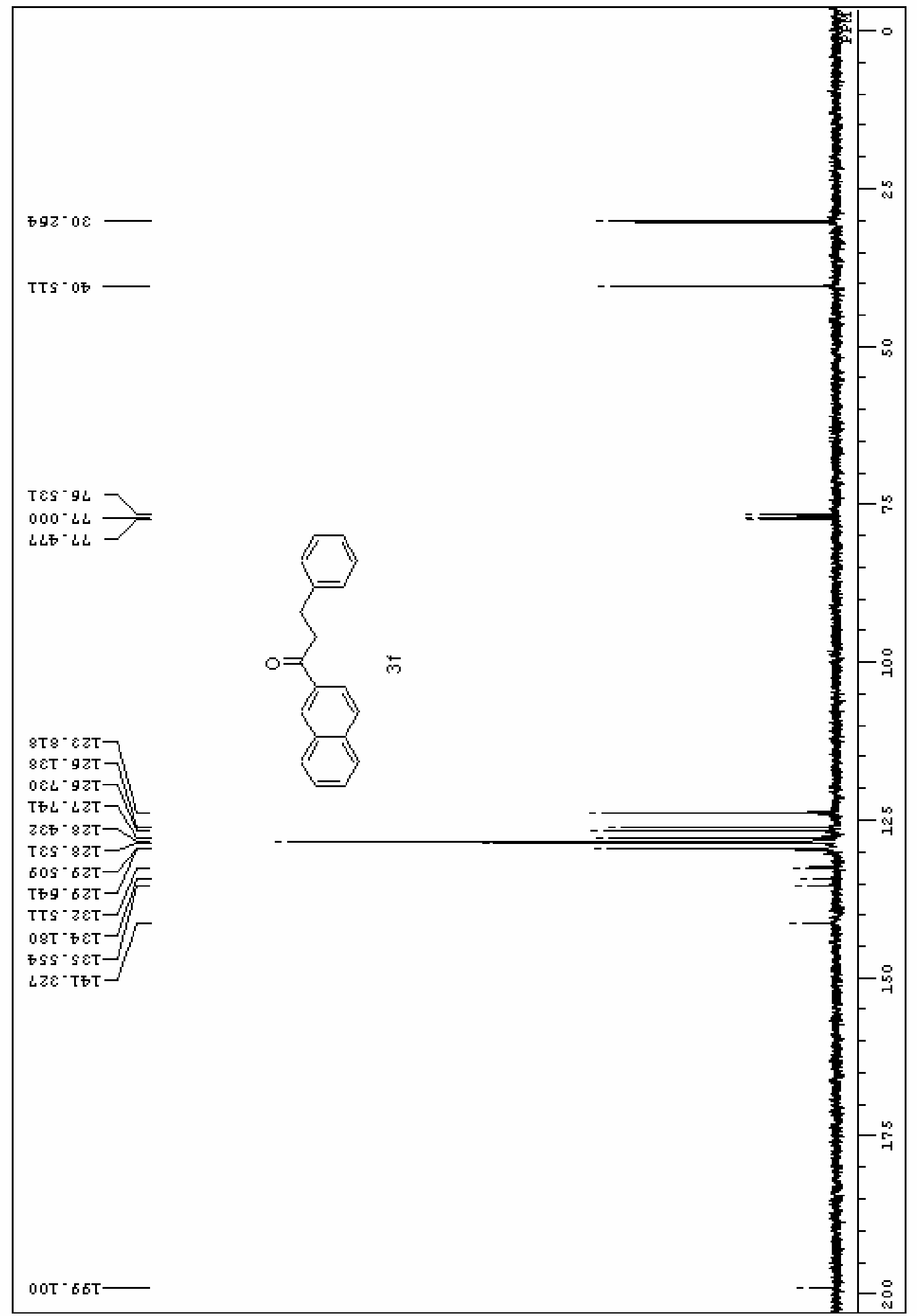




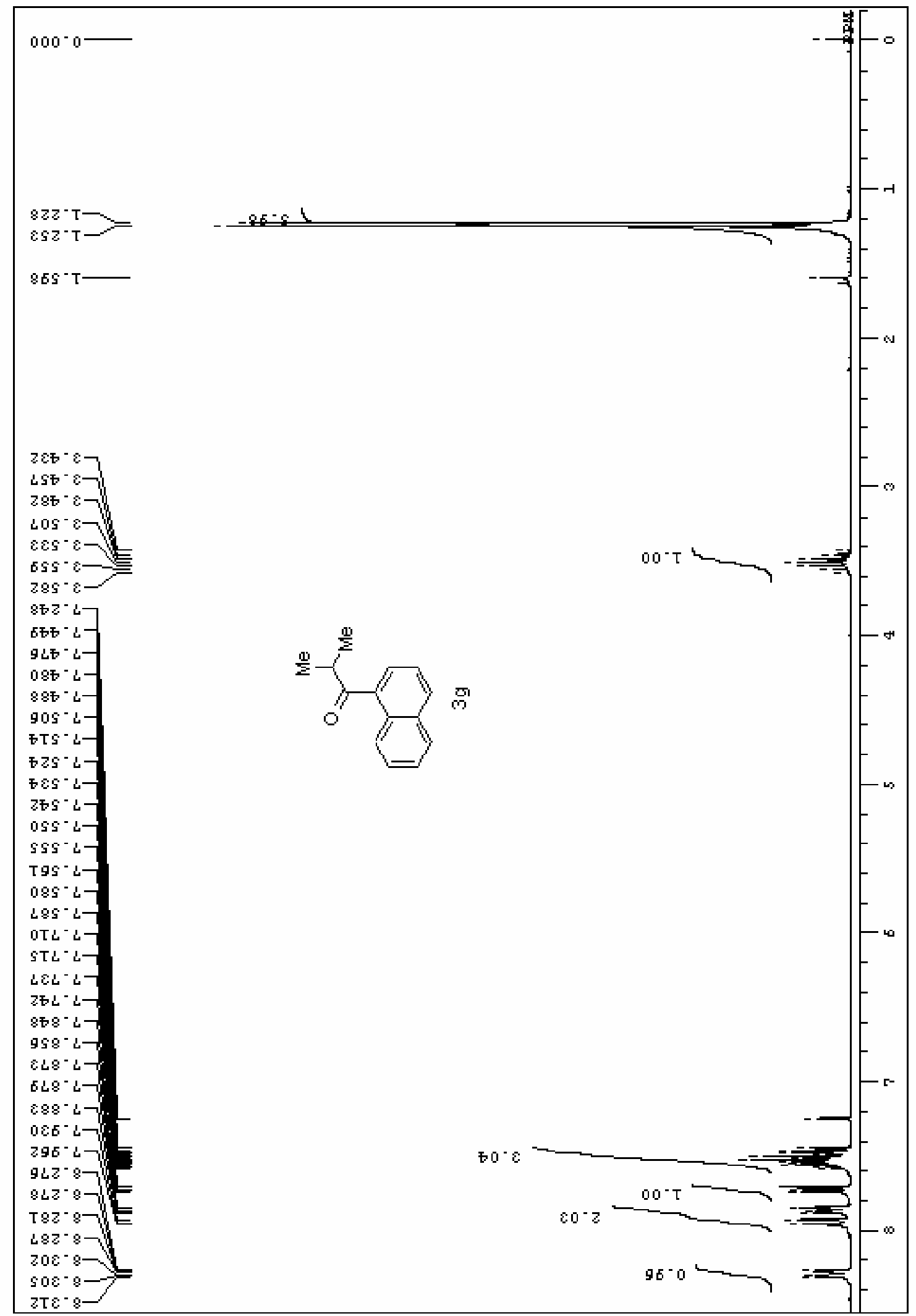




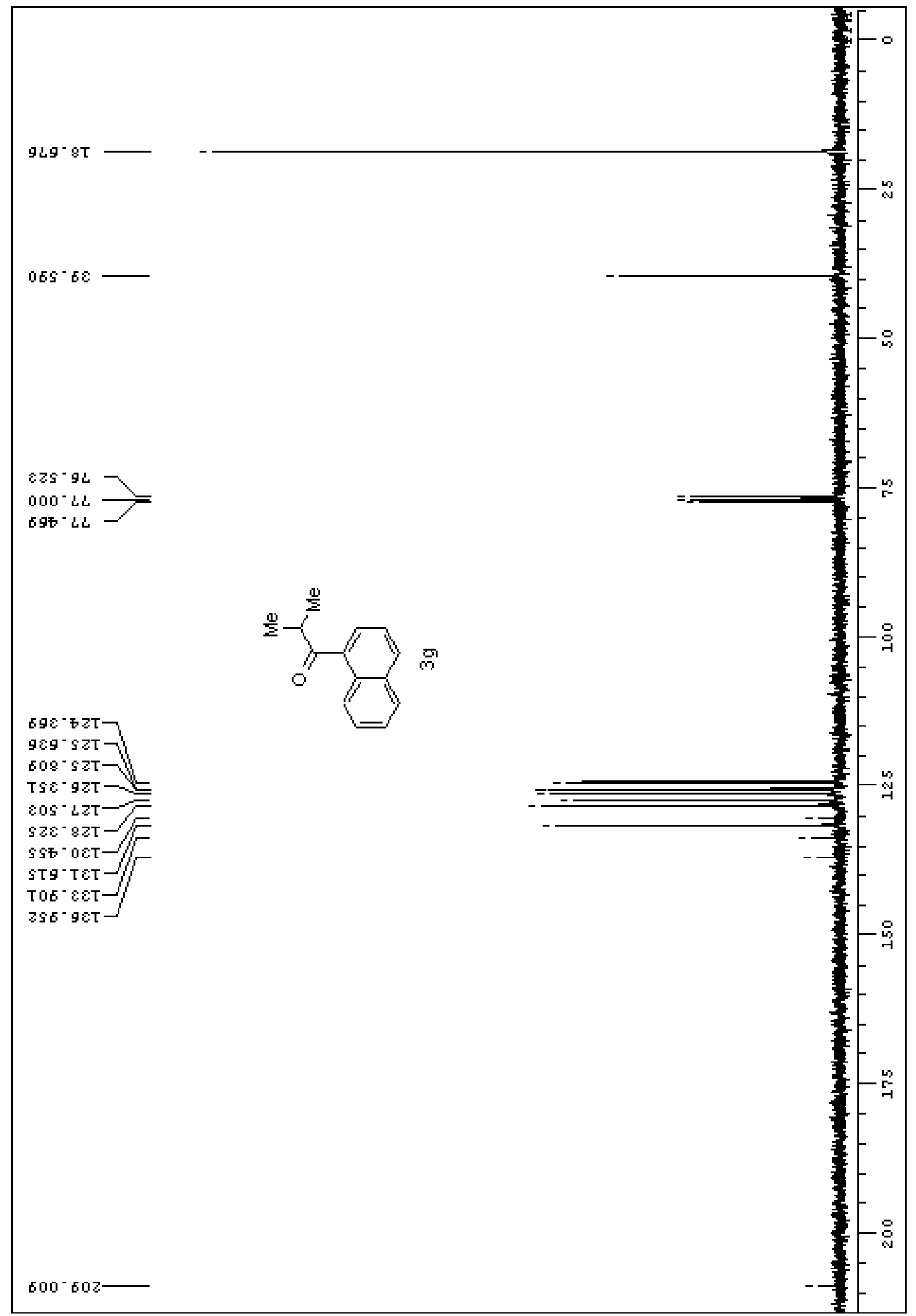




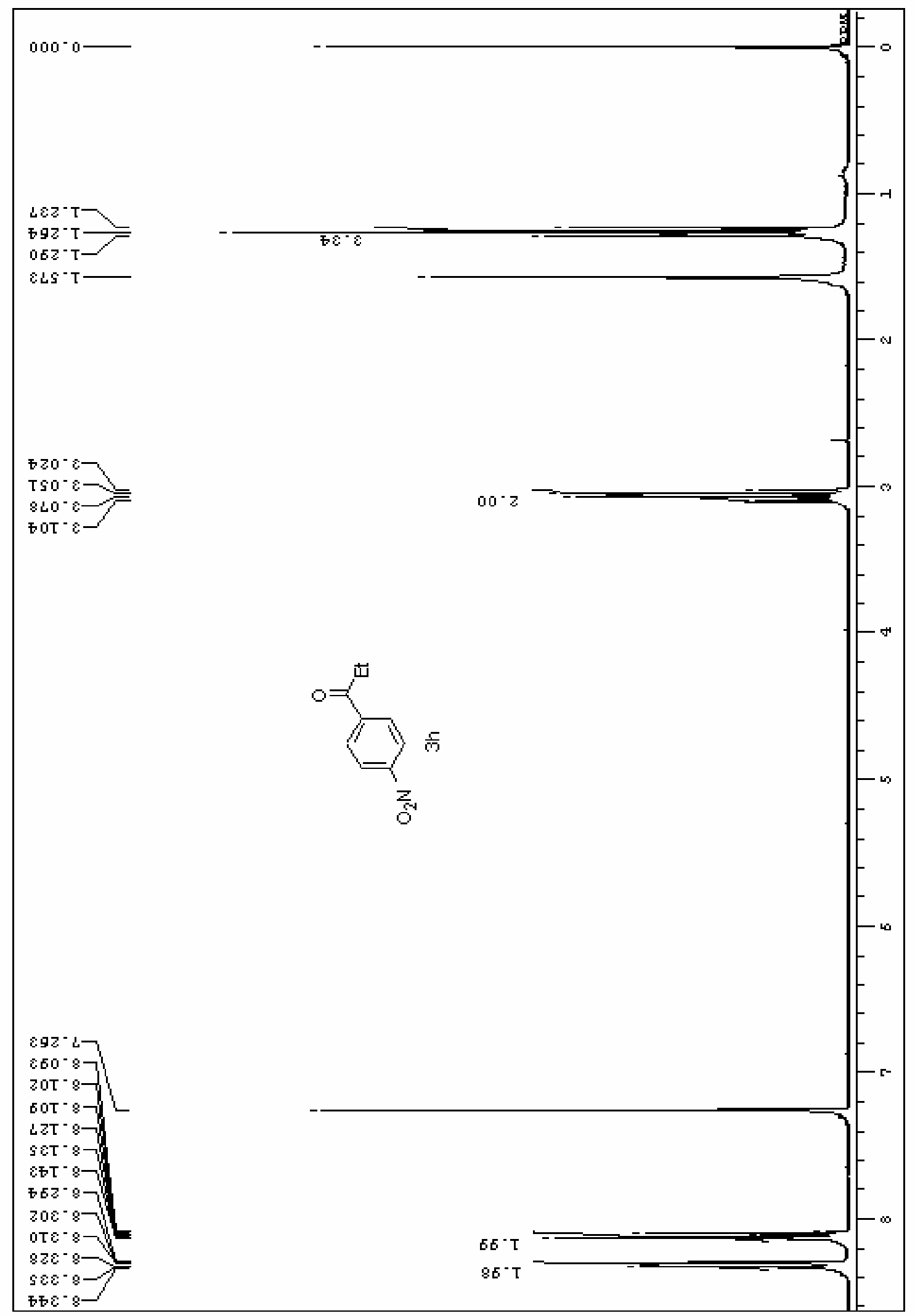




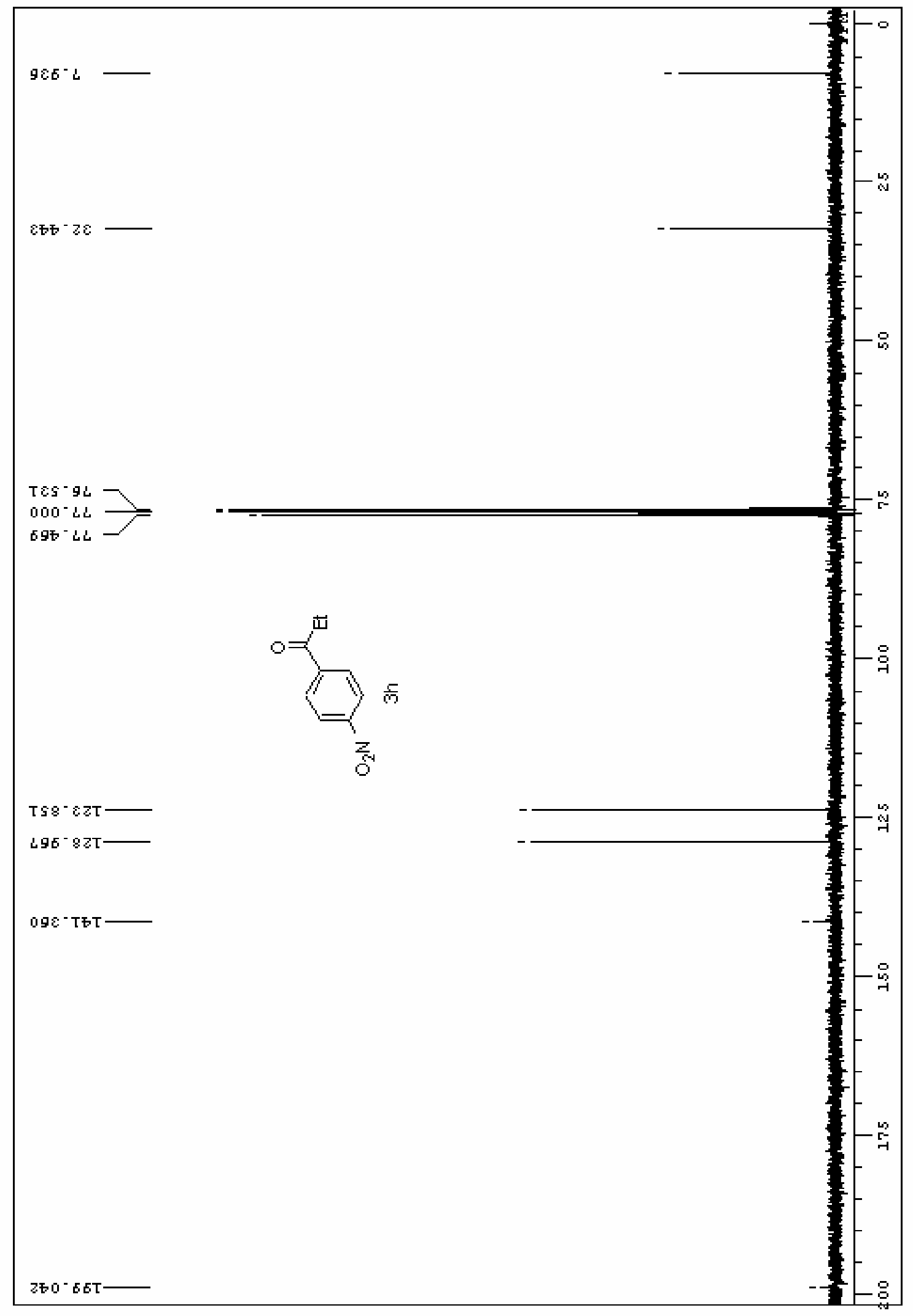




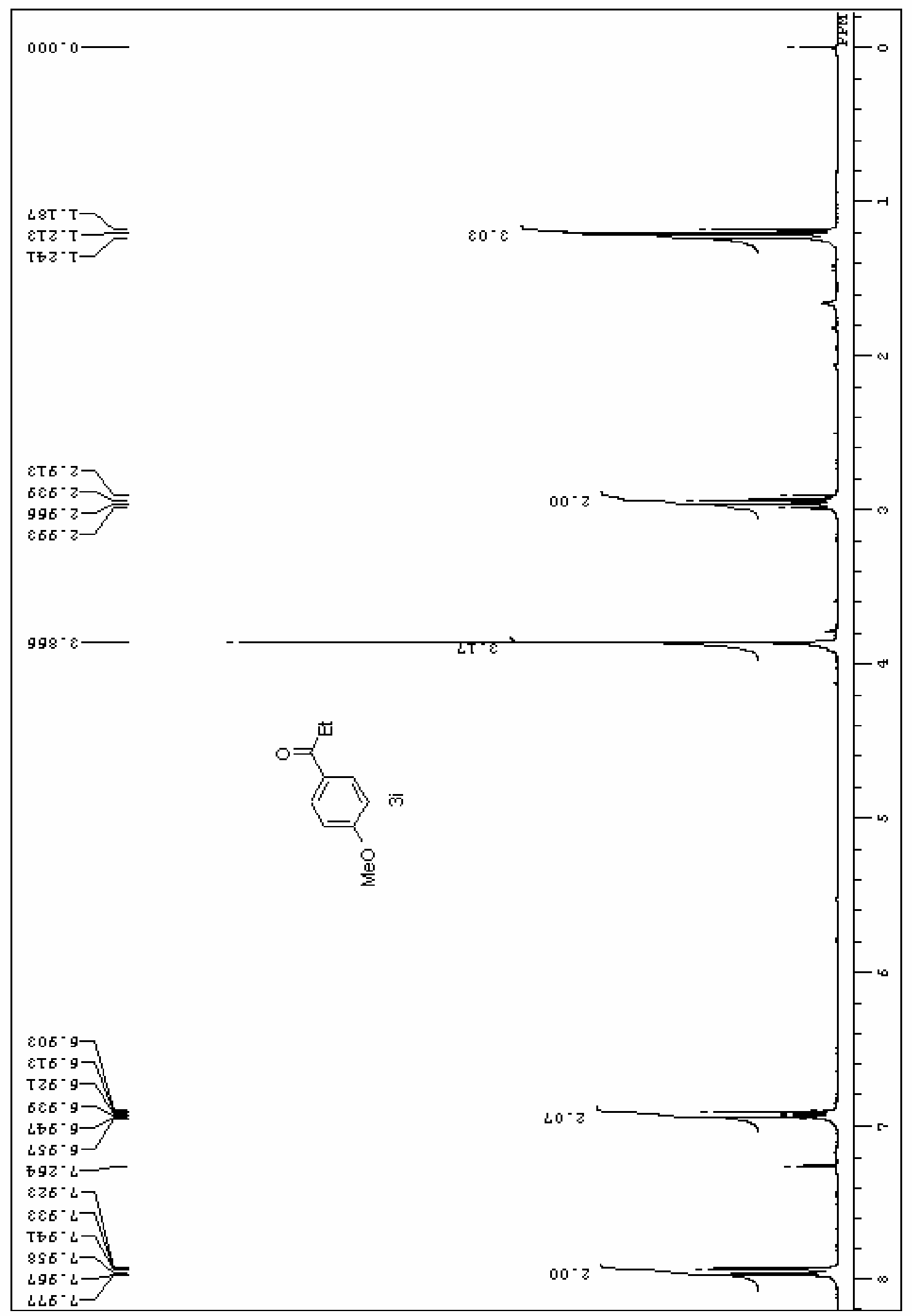




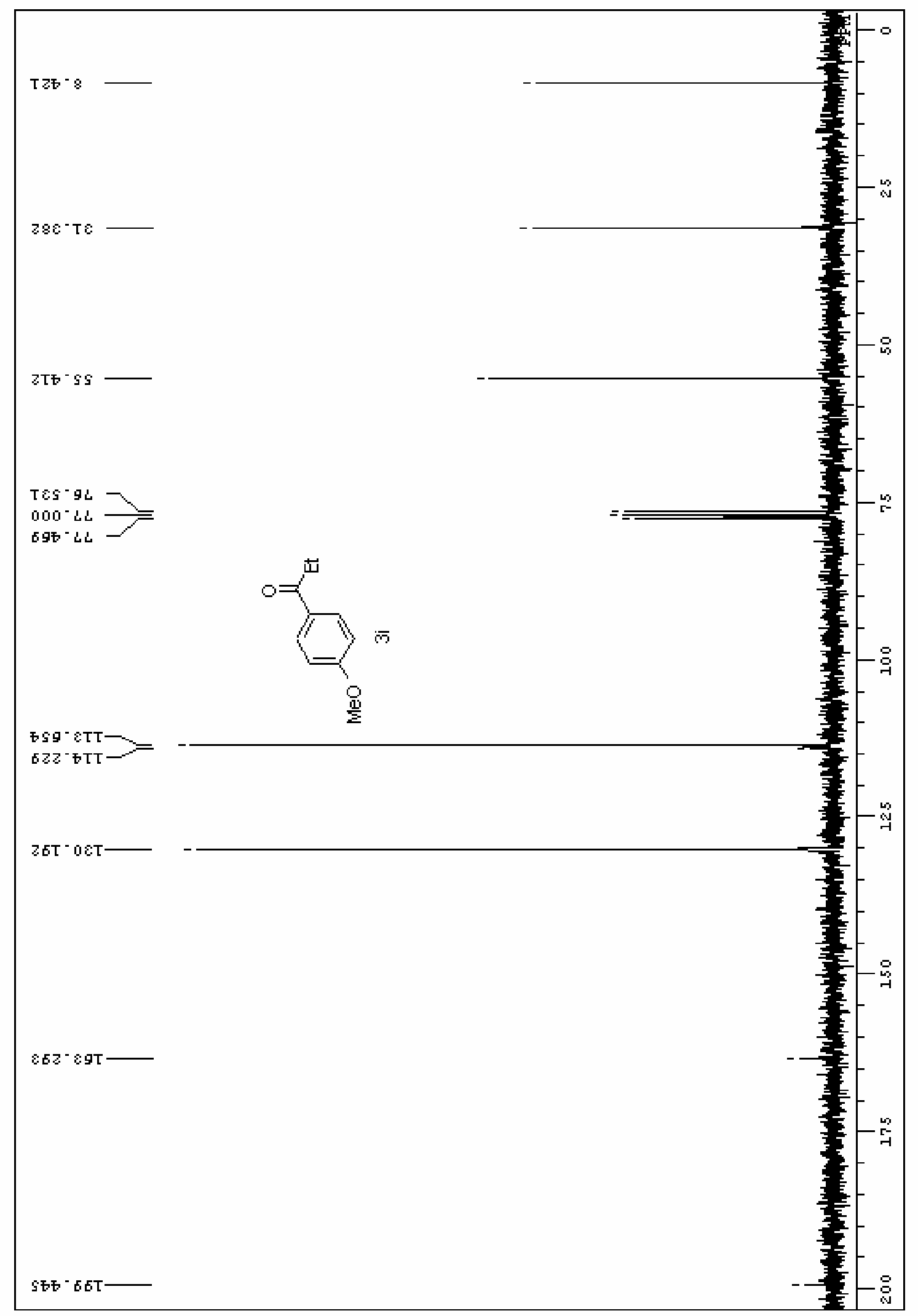




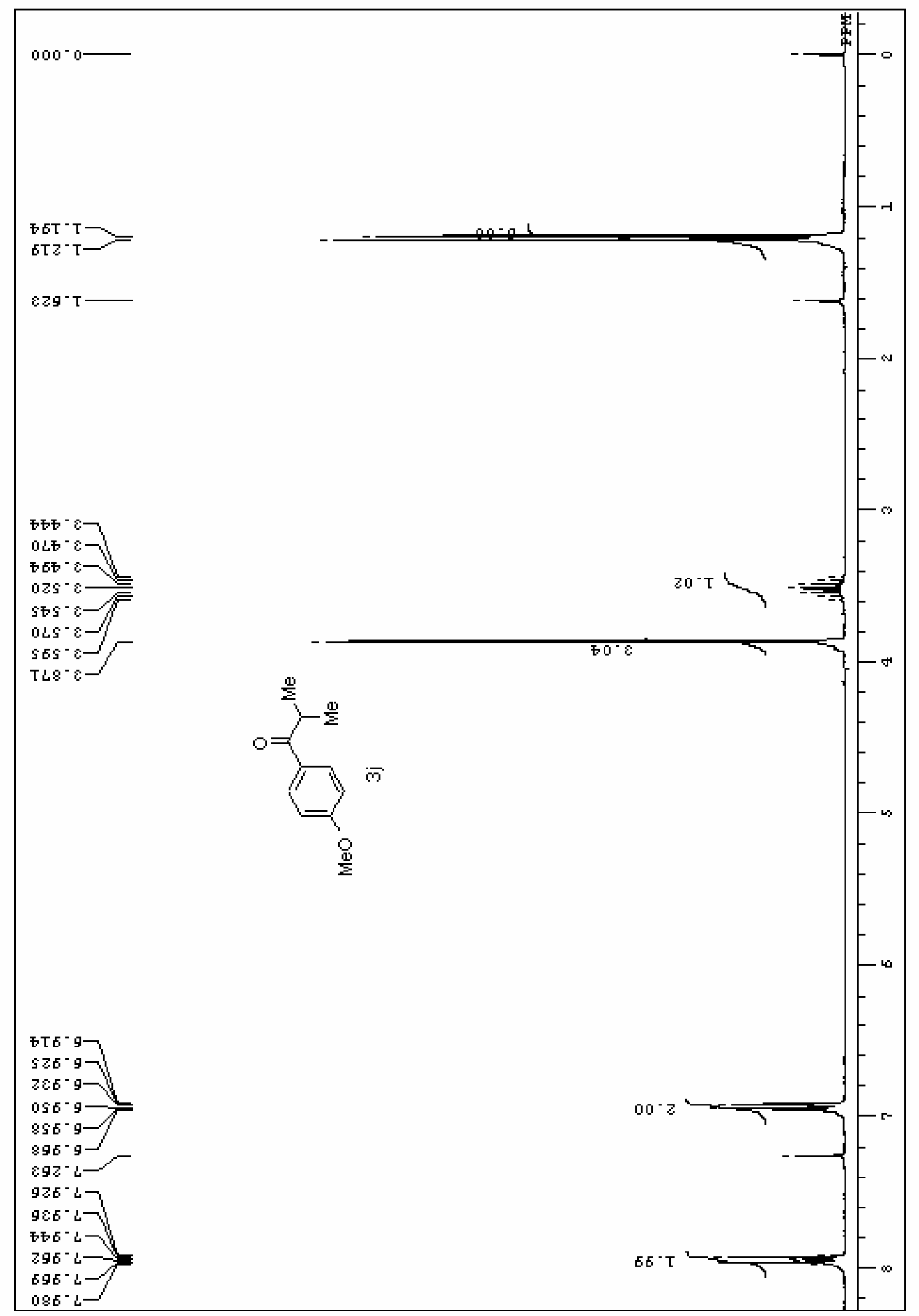




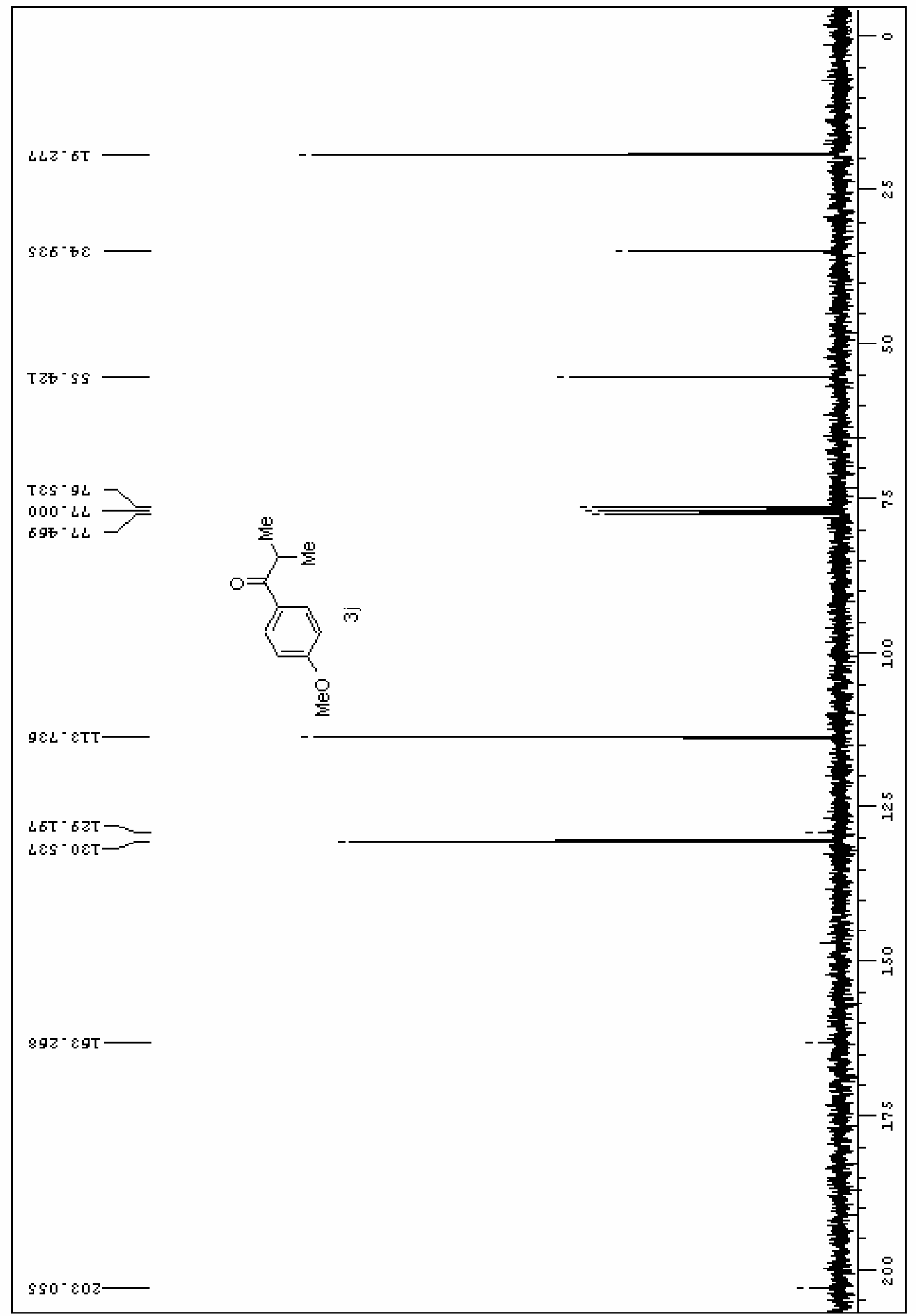




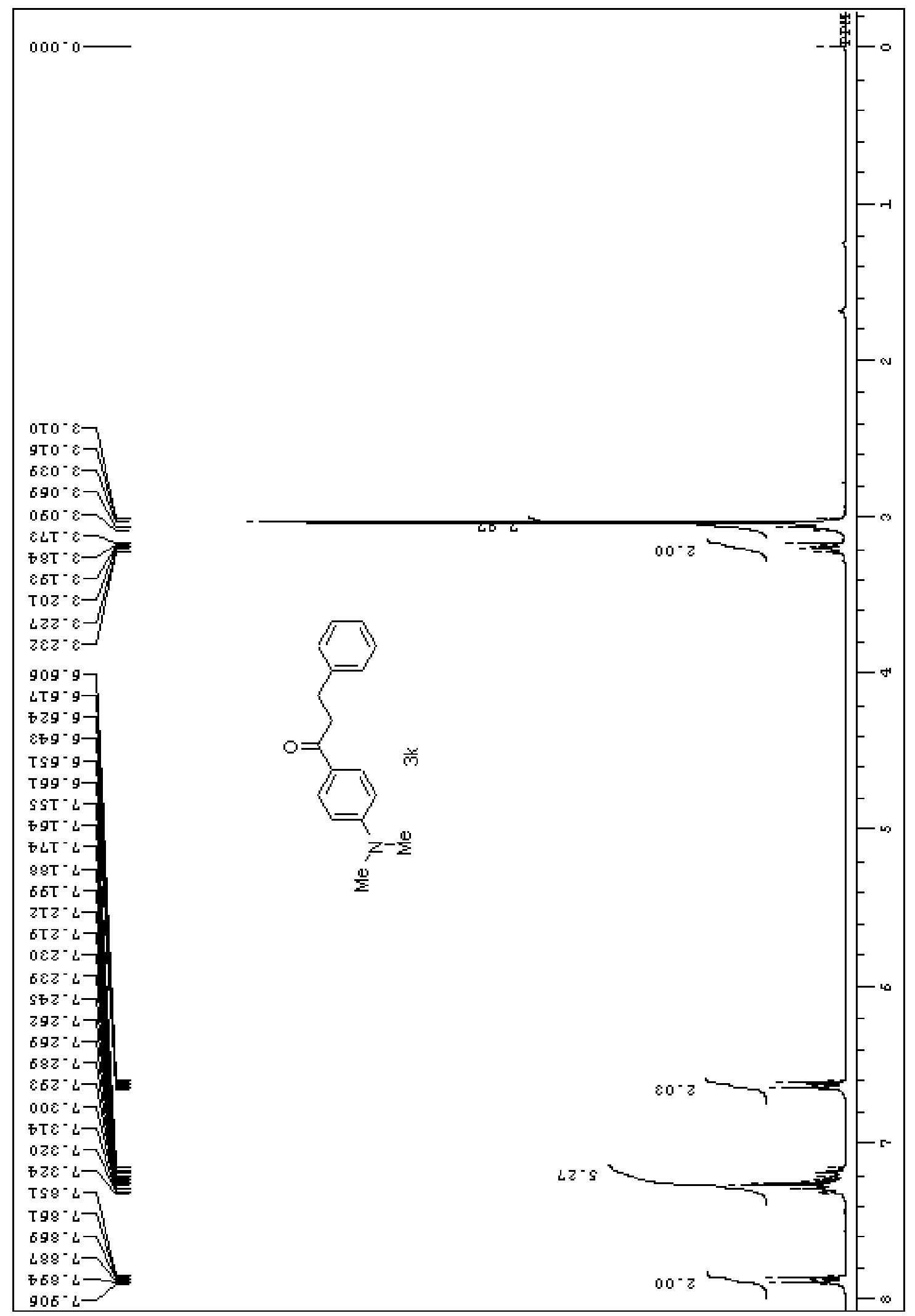




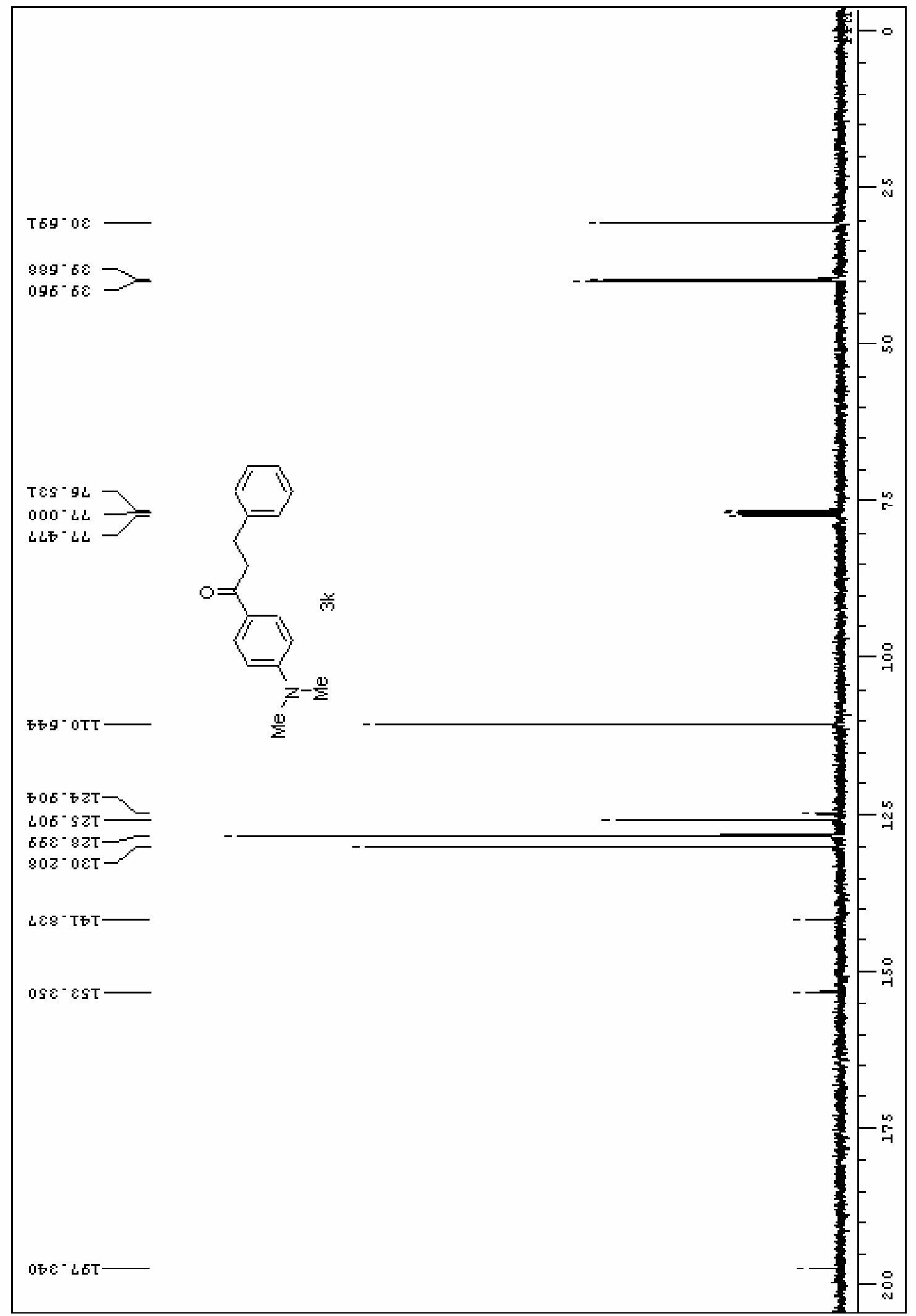




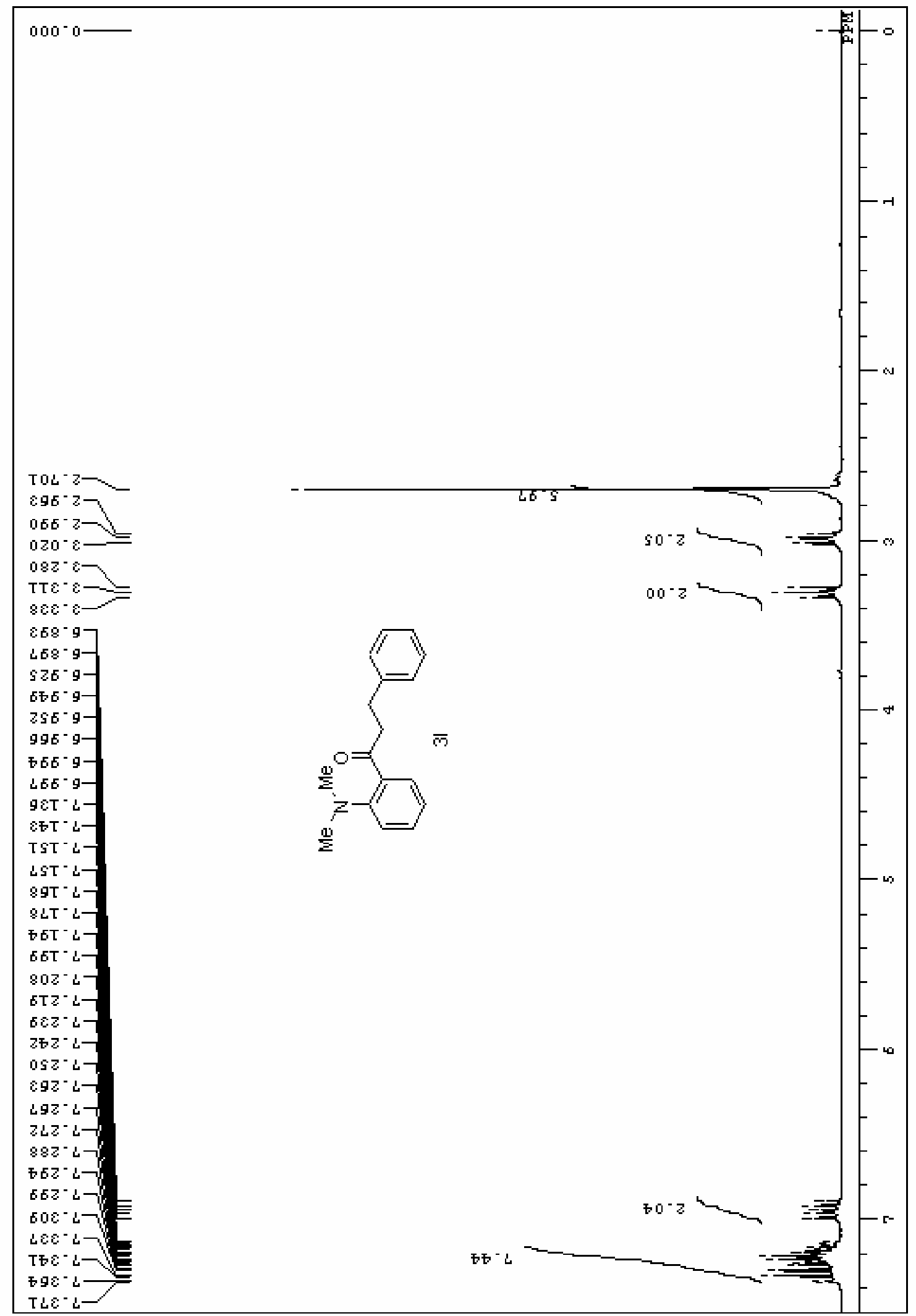




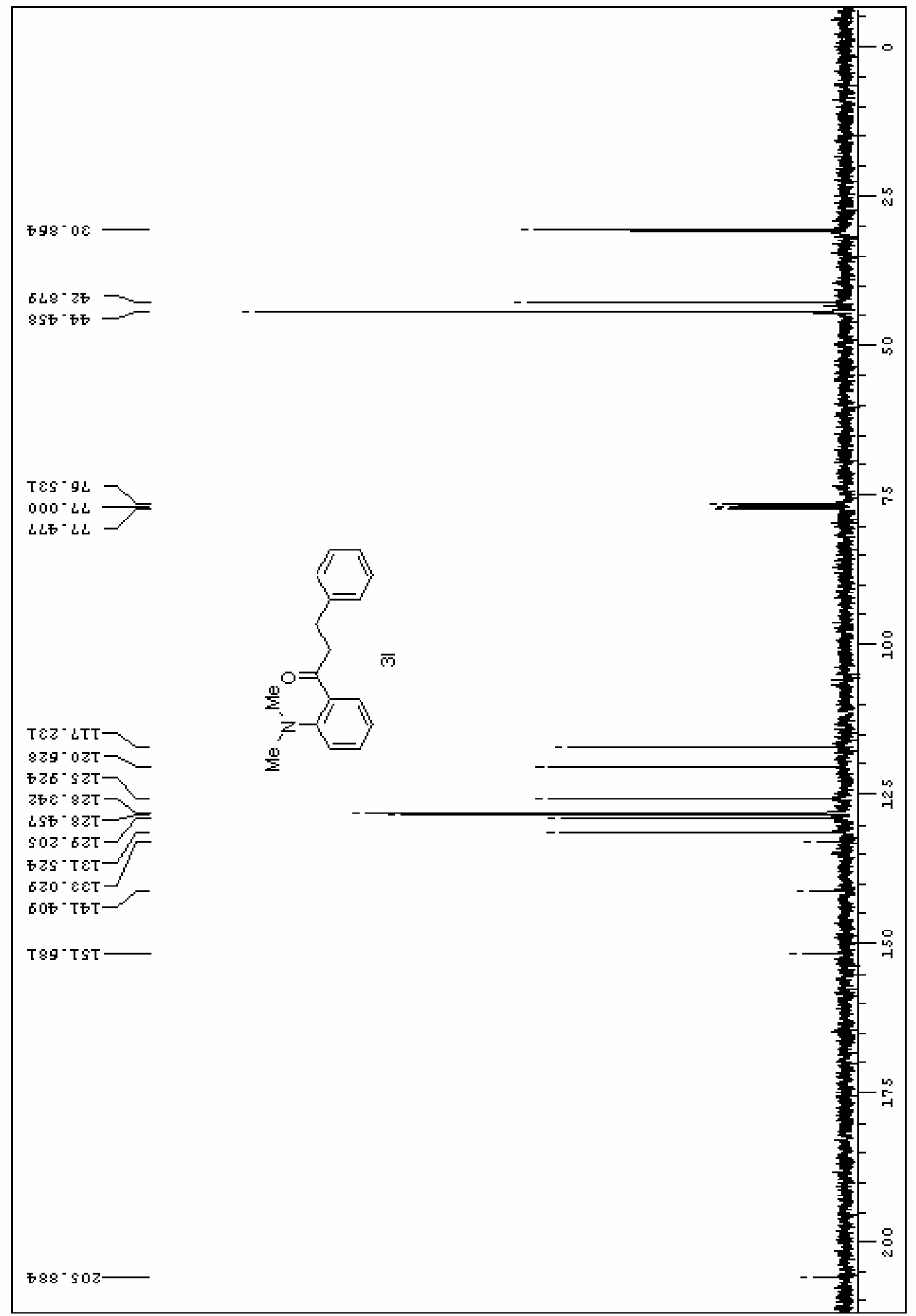




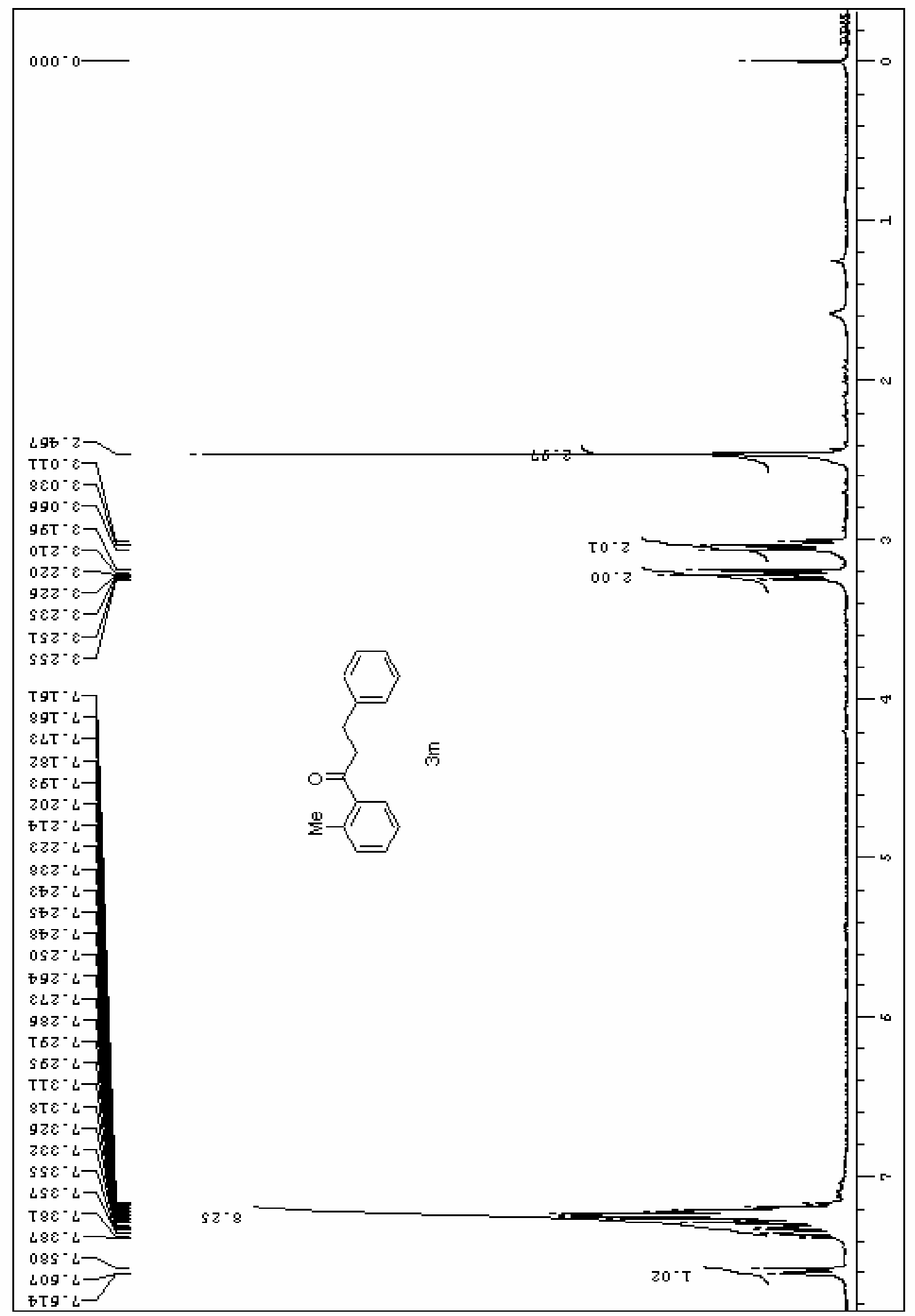




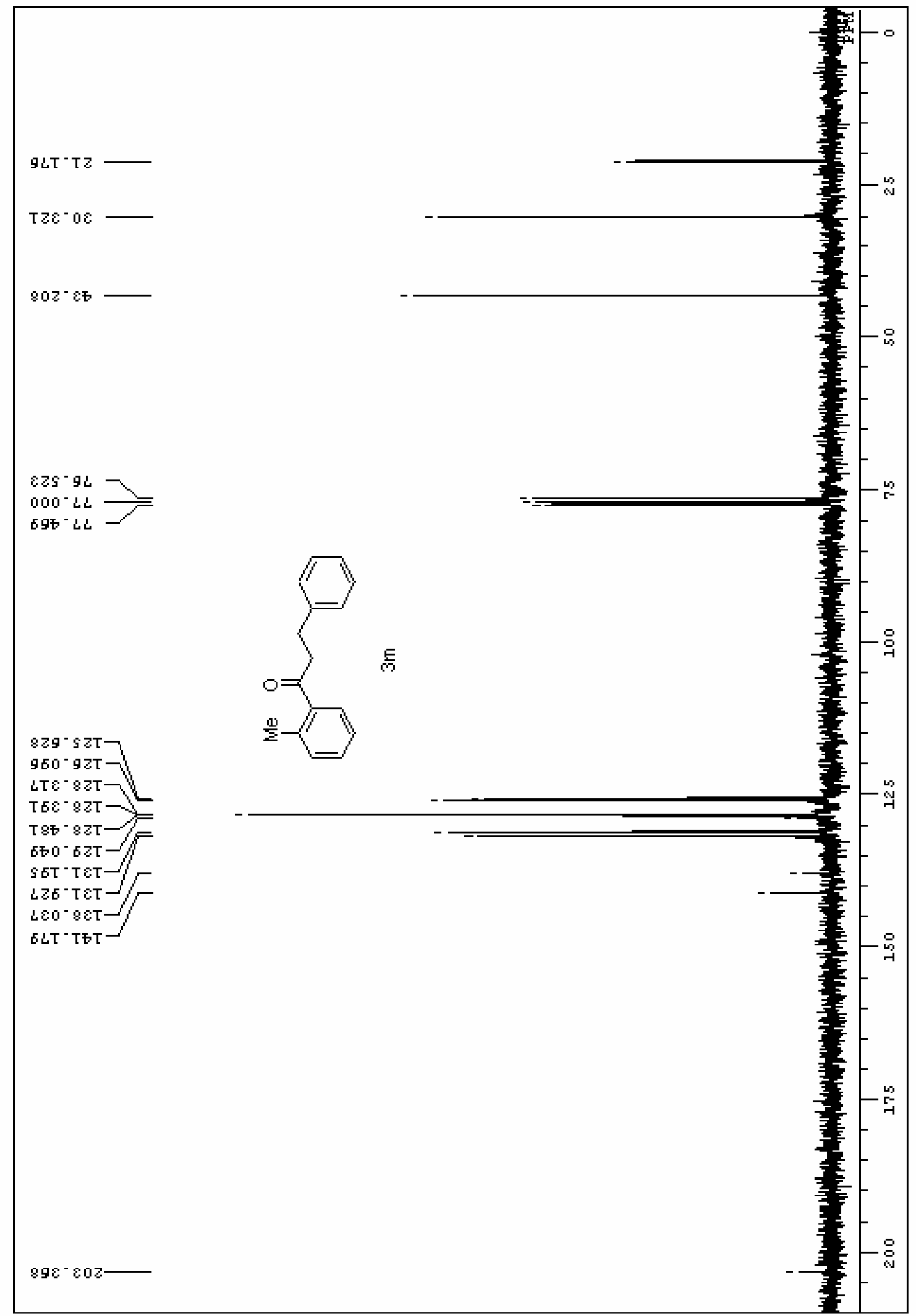




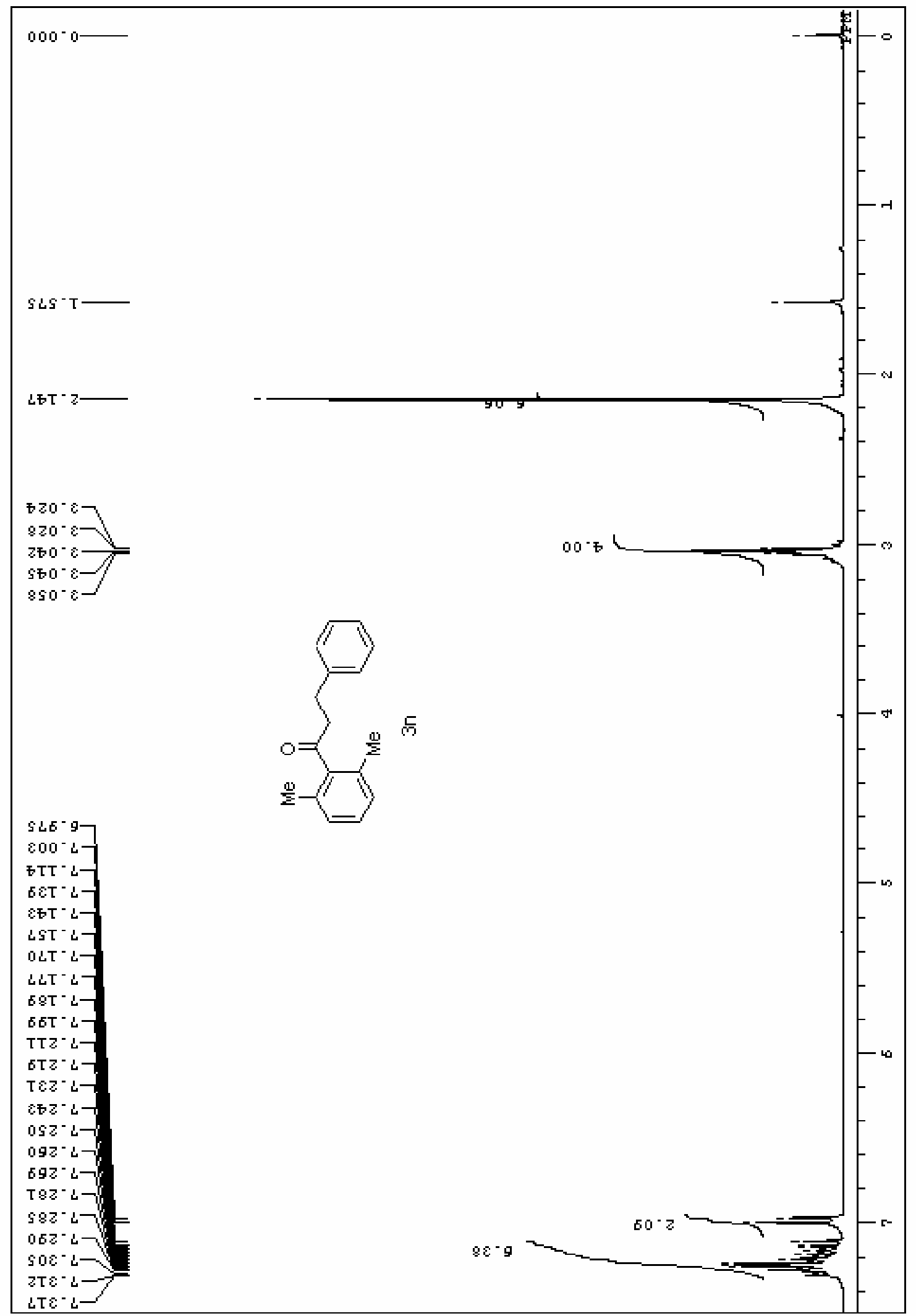




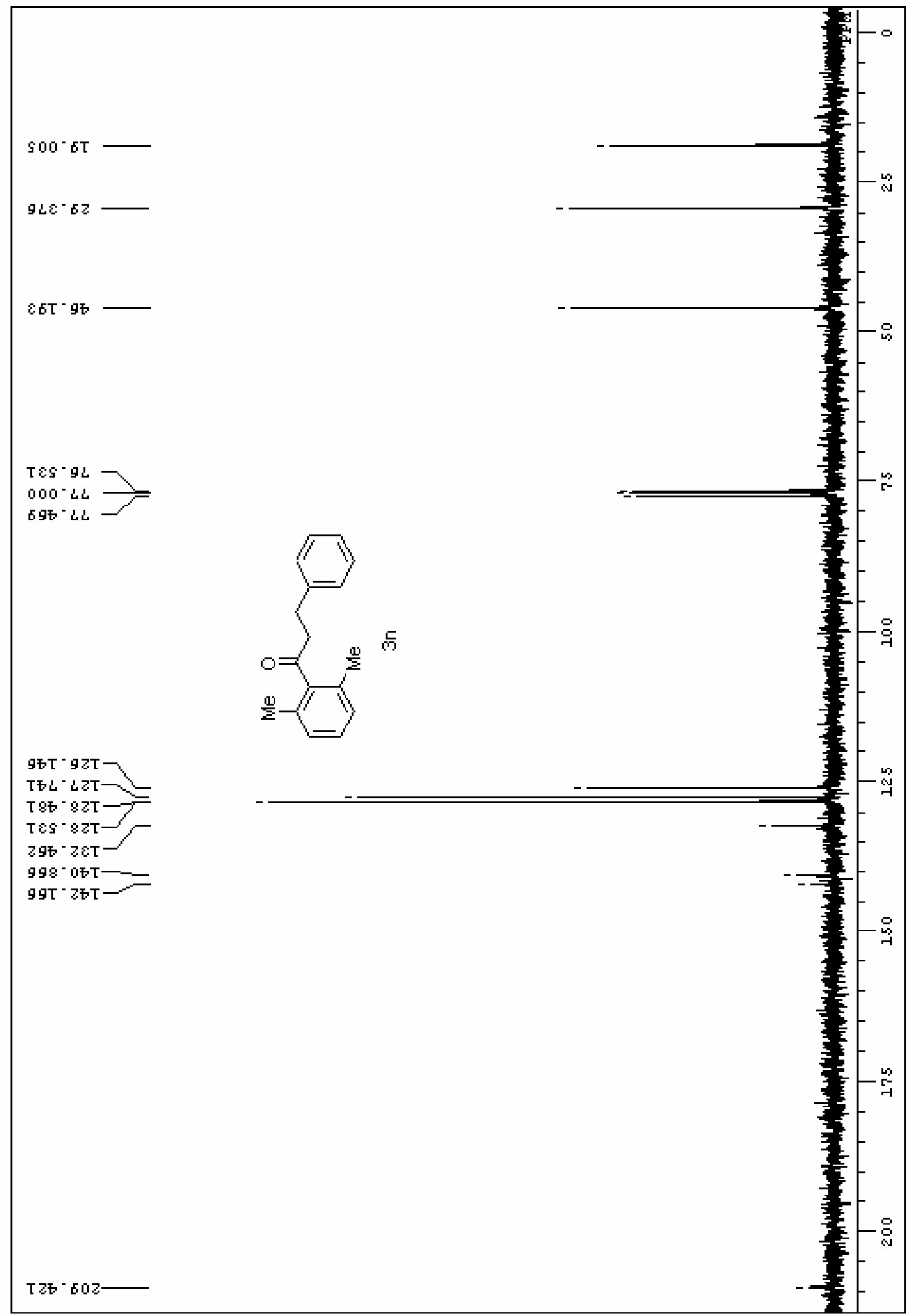




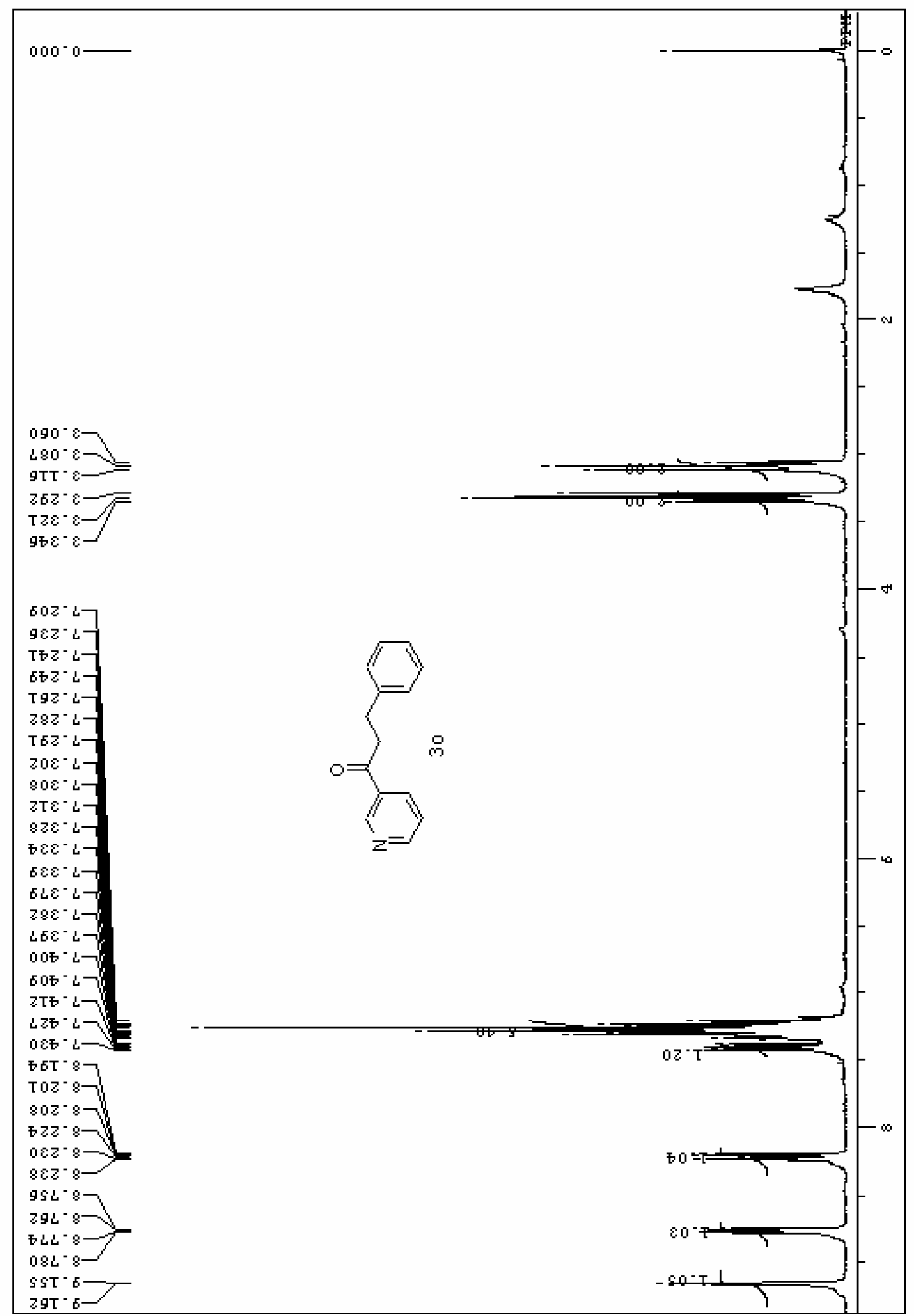




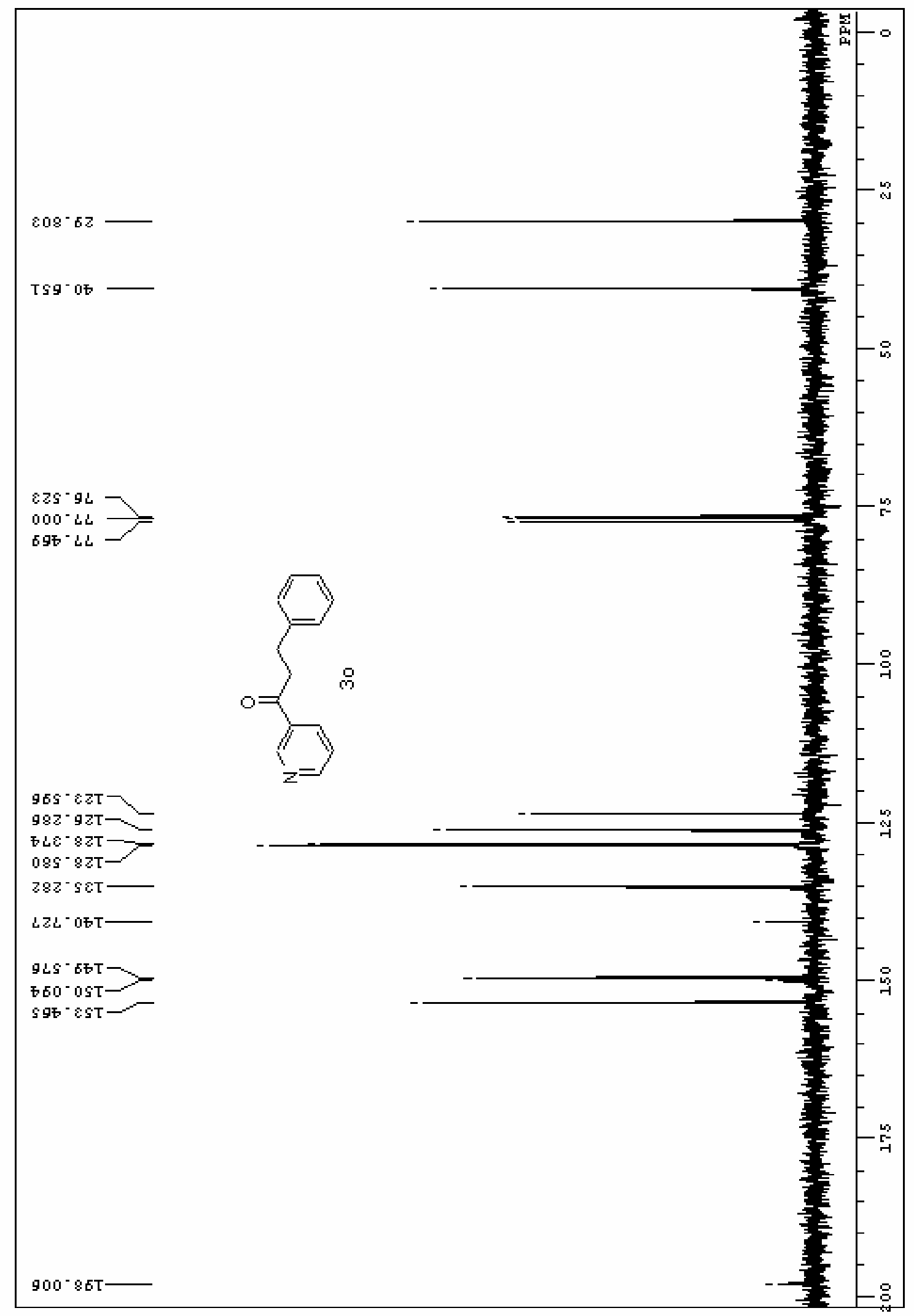




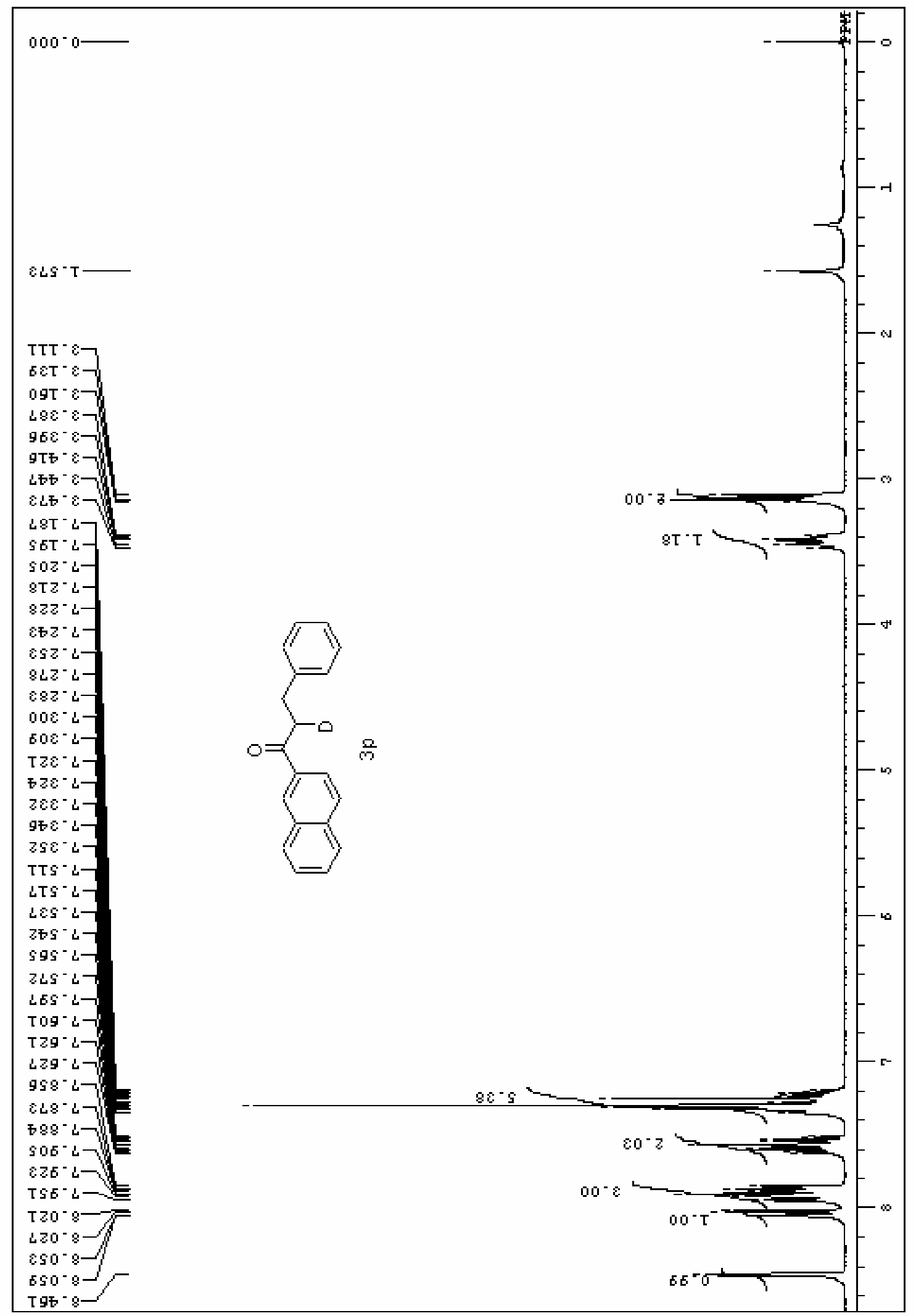




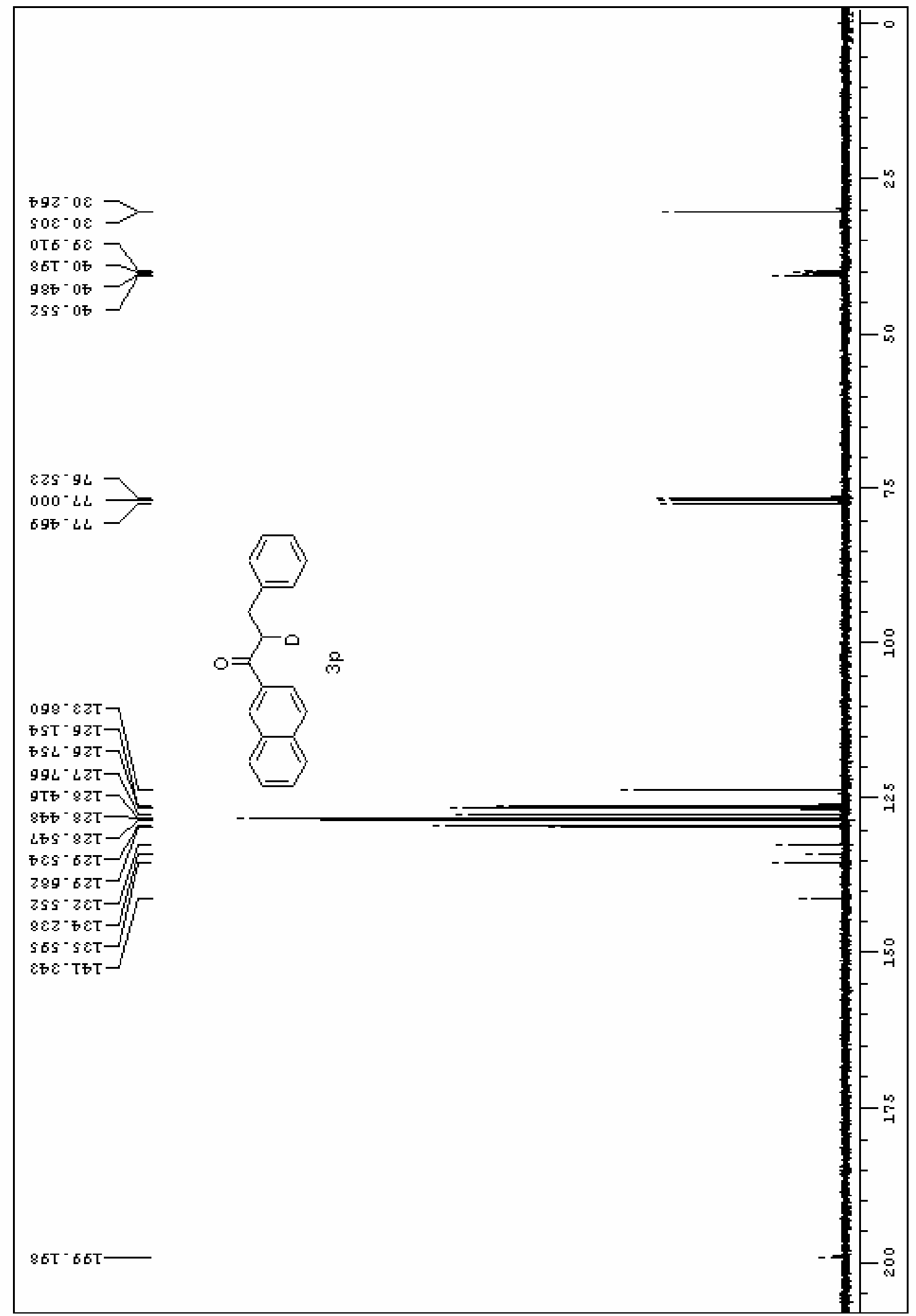

